Kardiologe $2021 \cdot 15: 542-584$

https://doi.org/10.1007/s12181-021-00504-6

Angenommen: 2. August 2021

Online publiziert: 21. September 2021

() Deutsche Gesellschaft für Kardiologie - Herzund Kreislaufforschung e.V. Published by Springer Medizin Verlag $\mathrm{GmbH}$, ein Teil von Springer Nature - all rights reserved 2021

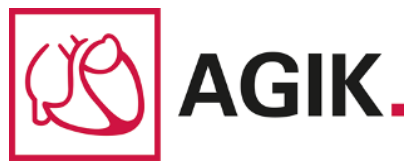

\title{
Manual der Arbeitsgruppe Interventionelle Kardiologie (AGIK) der Deutschen Gesellschaft für Kardiologie - Herz- und Kreislaufforschung e.V. (DGK)
}

\author{
Teil 2: „Durchführung der perkutanen Koronarintervention“
}

Holger M. Nef' · Stephan Achenbach ${ }^{2}$ Ralf Birkemeyer ${ }^{3}$ Alexander Bufe ${ }^{4,5}$. Oliver Dörr' $\cdot$ Albrecht Elsässer ${ }^{6} \cdot$ Luise Gaede $^{2} \cdot$ Tommaso Gori $^{7}$. Hans M. Hoffmeister ${ }^{8} \cdot$ Felix J. Hofmann $^{1} \cdot$ Hugo A. Katus ${ }^{9} \cdot$ Christoph Liebetrau $^{10,11}$. Steffen Massberg ${ }^{12} \cdot$ Matthias Pauschinger $^{13} \cdot$ Thomas Schmitz $^{14} \cdot$ Tim Süselbeck $^{15}$. Wolfram Voelker ${ }^{16}$. Jens Wiebe ${ }^{17}$. Ralf Zahnn ${ }^{18,19}$. Christian Hamm . Andreas M. Zeiher ${ }^{20} \cdot$ Helge Möllmann ${ }^{21}$

\section{Zusammenfassung}

Dieses Manual zur interventionellen Therapie (Teil 2) ist eine Anwendungsempfehlung für interventionell tätige Ärzte, die den gegenwärtigen Kenntnisstand unter Berücksichtigung neuester Studienergebnisse wiedergibt. Hierzu wurde in den einzelnen Kapiteln speziell auf die Alltagstauglichkeit der Empfehlungen geachtet, sodass dieses Manual jedem interventionell tätigen Kardiologen als Entscheidungshilfe im Herzkatheterlabor dienen soll. Trotz der von vielen Experten eingebrachten praktischen Hinweise kann dieses Manual dennoch nicht die ärztliche Evaluation des individuellen Patienten ersetzen und damit eine Anpassung der Diagnostik bzw. Therapie ersetzen.

\section{Schlüsselwörter}

Herzkatheterlabor · Koronare Herzerkrankung · Akutes Koronarsyndrom · Entscheidungshilfe · Herzkatheteruntersuchung

\section{Präambel und Einleitung}

Laut aktuellem Herzbericht 2018 erfolgten im Jahr 2017 an über 1000 Linksherzkathetermessplätzen in Deutschland ca. 880.000 Herzkatheteruntersuchungen. Entsprechend ergaben sich im Bundesdurchschnitt hochgerechnet 457 perkutane Koronarinterventionen auf 100.000 Personen, d. h. 42,9\% der Herzkatheteruntersuchungen umfassen eine perkutane Koronarintervention $(\mathrm{PCl})$ [1].

Angesichts dieser Vielzahl an Prozeduren erscheint es der Arbeitsgruppe Interventionelle Kardiologie (AGIK) im Auftrag der Deutschen Gesellschaft für Kardiologie - Herz- und Kreislaufforschung e. V. (DGK) umso wichtiger, eine möglichst standardisierte Vorgehensweise zu etablieren - dies zum einen vor dem Hintergrund der stetig steigenden Komplexität der Patientenfälle und zum anderen, um dem Qualitätsanspruch an eine moderne Diagnostik bzw. Intervention gerecht zu werden.

Dieses Manual zur diagnostischen Herzkatheteruntersuchung (Teil 1) [2] und zur perkutanen Koronarintervention (Teil 2) ist deshalb eine Anwendungsempfehlung für interventionell tätige Ärzte, die den gegenwärtigen Kenntnisstand unter Berücksich- 
tigung neuester Studienergebnisse wiedergibt. Hierzu wurde in den einzelnen Kapiteln speziell auf die Alltagstauglichkeit der Empfehlungen geachtet, sodass dieses Manual jedem interventionell tätigen Kardiologen als Entscheidungshilfe im Herzkatheterlabor dienen soll. Es kann selbstverständlich nicht die ärztlicheEvaluation des individuellen Patienten ersetzen und damit die Anpassung der Diagnostik bzw. der Therapie an dessen spezifische Situation.

Dieses Manual ist die zweite erweiterte Auflage der bereits im Jahr 2008 publizierten Positionspapiere [3, 4]. Bei der Erstellung erfolgte eine systematische Analyse der vorhandenen Literatur und der besten verfügbaren wissenschaftlichen Evidenz. Dies ist nach dem gängigen Schema der Empfehlungsgrade innerhalb des Manuals entsprechend gekennzeichnet:

\section{Empfehlungsgrade}

- I: Evidenz und/oder allgemeine Übereinkunft, dass eine Therapieform oder eine diagnostische Maßnahme effektiv, nützlich oder heilsam ist (wird empfohlen/ist indiziert)

- II: Widersprüchliche Evidenz und/oder unterschiedliche Meinungen über den Nutzen/Effektivität einer Therapieform oder einer diagnostischen Maßnahme

- Ila: Evidenzen/Meinungen favorisieren den Nutzen bzw. die Effektivität einer Maßnahme (sollte erwogen werden)

- Ilb: Nutzen/Effektivität einer Maßnahme ist weniger gut durch Evidenzen/ Meinungen belegt (kann erwogen werden)

- III: Evidenz und/oder allgemeine Übereinkunft, dass eine Therapieform oder eine diagnostische Maßnahme nicht effektiv, nicht nützlich oder nicht heilsam ist und im Einzelfall schädlich sein kann (wird nicht empfohlen)

\section{Evidenzgrade}

- A: Daten aus mehreren randomisierten klinischen Studien oder Metaanalysen

- B: Daten aus einer randomisierten klinischen Studie oder mehreren großen nicht randomisierten Studien

- C: Konsensusmeinung von Experten und/oder kleinen Studien, retrospektiven Studien oder Registern
Grundlage dieses Manuals waren insbesondere die Europäischen Leitlinien zur myokardialen Revaskularisation in der Version von 2018 sowie die korrespondierenden ACC/AHA-Richtlinien [5, 6]. Ergänzt ist dieses Manual durch praktische Tipps zum prozeduralen Vorgehen, welche die Auffassungen verschiedener „Katheterschulen“ wiedergeben und im Konsens der Autorengruppe verfasst wurden. Richtlinien zur Einrichtung und für die Betreibung von Herzkatheterlaboren wurden für Deutschland separat publiziert [7].

\section{Allgemeines praktisches Vorgehen bei der $\mathrm{PCl}$}

\subsection{Vorbereitung der $\mathrm{PCl}$}

Grundsätzlich gehört zur PCI-Vorbereitung die Überprüfung aller Voraussetzungen, die auch für eine diagnostische Angiographie gegeben sein müssen (s. Kap. 1 des Beitrags Manual der Arbeitsgruppe Interventionelle Kardiologie [AGIK] der Deutschen Gesellschaft für Kardiologie - Herz- und Kreislaufforschung e.V. [DGK]. Teil 1: „Durchführung der diagnostischen Herzkatheteruntersuchung" [2]). Insbesondere sind das Vorhandensein von Begleiterkrankungen (Niereninsuffizienz, Anämie, Schilddrüsenfunktionsstörungen, Gerinnungsstörungen, Diabetes mellitus, Infektionen, Allergien u.a.), die Medikamentenanamnese sowie die vorliegenden Laboruntersuchungen zu berücksichtigen. Strukturierte Protokolle für die Erfassung und das periprozedurale Management von Komorbiditäten sind zu empfehlen (s. Kap. 5 und 7 des Beitrags Manual der Arbeitsgruppe Interventionelle Kardiologie [AGIK] der Deutschen Gesellschaft für Kardiologie - Herz- und Kreislaufforschung e.V. [DGK]. Teil 1: „Durchführung der diagnostischen Herzkatheteruntersuchung" [2]).

Darüber hinaus muss eine adäquate vom Patienten unterzeichnete Aufklärung vorliegen. Üblicherweise wird bei der Aufklärung für die diagnostische Koronarangiographie auch die Einverständniserklärung für eine evtl. perkutane Koronarintervention eingeholt. Dies erlaubt die Durchführung einer $\mathrm{Ad}$-hoc-PCl bei hierfür geeigneten Patienten. Die Durchführung einer Ad-hoc-PCl ist aber insbesondere bei elektiven Eingriffen an das Vorliegen weiterer Voraussetzungen gebunden. Hierzu gehört zum einen eine suffiziente Ischämiediagnostik, zum anderen sollten ein Erkrankungsausmaß und eine Koronarmorphologie vorliegen, bei denen das lokale Heart-Team üblicherweise ein perkutanes Vorgehen empfiehlt (s. Kap. 3). Idealerweise sind solche Ad-hoc-PCl-Szenarien in einer vom lokalen Heart-Team abgefassten SOP beschrieben. Vor komplexen perkutanen Eingriffen und in Situationen, in denen eine Bypassoperation als alternative Behandlungsoption diskutiert werden sollte, empfiehlt es sich, den Patienten zunächst abzulegen und inn erneut über Nutzen und Risiken des Eingriffs sowie alternative Vorgehensweisen aufzuklären (ggf. auch durch den Herzchirurgen). Danach sollte inm eine ausreichende Bedenkzeit eingeräumt werden [6].

Der eigentliche Eingriff wird dann am ausreichend hydrierten Patienten durchgeführt. Eine Nahrungskarenz ist nicht zwingend erforderlich, ggf. nur bei Patienten mit antizipierbarer komplexer Intervention. Für die Untersuchung müssen ein intakter venöser Zugang sowie ein kontinuierliches EKG-Monitoring gewährleistet sein. Zusätzlich muss die Möglichkeit einer oxymetrischen Überwachung gegeben sein. Die Nierenfunktion des Patienten ist in die Planung des Eingriffs mit einzubeziehen; dabei ist auch die bereits diagnostisch verabreichte Kontrastmittelmenge zu berücksichtigen. Bei Bedarf und auf Wunsch des Patienten ist die orale oder intravenöse Gabe eines schnell wirksamen Sedativums möglich. Eine ausreichende Überwachung des Patienten während des gesamten Eingriffs muss gewährleistet sein. Eine Vollnarkose ist in der Regel nicht gerechtfertigt. Bei Patienten, die noch keine duale Plättchenhemmung erhalten haben, sollte diese eingeleitet werden, sobald die Entscheidung zur $\mathrm{PCl}$ getroffen wird. Perkutane Interventionen erfordern regelhaft eine periprozedurale Antikoagulation. Einzelheiten der antithrombotischen Therapie sind in Kap. 5 beschrieben.

\subsection{Führungskatheter}

Grundsätzlich gilt, dass die KathetergröBe bzw. Katheterform so gewählt werden 
sollte, dass möglichst eine koaxiale Ausrichtung zwischen Katheter und Koronarostium gewährleistet ist. Ebenfalls ist ein der Prozedur angepasstes Back-up bei der Auswahl des Führungskatheters zu beachten. In den meisten Fällen ist die Durchführung der $\mathrm{PCl}$ mit einem 6 F-Katheter möglich. Bei Verwendung großkalibriger Devices (z.B. Rotablator) ist im Einzelfall die Durchführung der Prozedur mit einem $7 \mathrm{~F}$ - oder 8 F-Führungskatheter zu prüfen.

Für die $\mathrm{PCl}$ des linken Koronarsystems sollte ein Extra-Back-up-Katheter (XB oder EBU) verwendet werden. Ausnahme kann eine Läsion im Bereich des ostialen Hauptstamms darstellen.

Für die $\mathrm{PCl}$ des rechten Koronarsystems kann ein Großteil der Prozeduren (insbesondere bei Ostiumstenosen) mit einem Judkins-Katheter durchgeführt werden. Bei komplexen Prozeduren (starke Kalzifizierung und distale Läsionen) kann ein Amplatz-Left-Katheter (AL 0,75 oder 1,0) Vorteile bieten.

Hinsichtlich des radialen Gefäßzugangs ergeben sich folgende Besonderheiten: Aufgrund des dünneren Gefäßkalibers der A. radialis unterscheiden sich die Auswahl und die Manipulation der Führungskatheter im Vergleich zum femoralen Zugang. Dennoch können auch radial die meisten Koronarinterventionen mit einem 6 FFührungskatheter durchgeführt werden. In Einzelfällen kann die Verwendung von 7 F-Kathetern möglich sein. Es ist darauf zu achten, dass die Kurvengröße des Führungskatheters für gewöhnlich 0,5 kleiner gewählt wird als beim femoralen Zugang (z.B. EBU 4,0 $\rightarrow$ EBU 3,5; JL 4,0 $\rightarrow$ JL 3,5).

\subsection{Ballonsysteme}

Die Behandlung von Koronarstenosen durch eine Ballondilatation ist seit dem ersten Einsatz eines Ballonkatheters im Koronarsystem durch Grüntzig 1977 unverändert die grundlegende Technik der Koronarintervention geblieben.

Die frühere OTW(„over the wire ${ }^{\prime)}$ Konstruktion wurde weitestgehend durch Monorailsysteme abgelöst [8]. OTW-Ballons haben noch eine Nischenindikation im Rahmen der TASH(transkoronare Ablation der Septumhypertrophie)-Behandlung. Bei der Behandlung von chronischen Verschlüssen wurden sie weitgehend durch
Mikrokatheter ersetzt. Die wesentlichen Performance-Kriterien eines Ballonsystems sind die "Trackability" (Passage von Gefäßwindungen und Abzweigungen), die „Pushability" (Übertragung des Vorwärtsschub vom Ende auf die Spitze) und die "Crossability" (Passage von Stenosen). Diese Eigenschaften hängen unter anderem von Ballonmaterial und-durchmesser, dem Aufbau und der Härte des Ballonschafts, den aufgebrachten Beschichtungen, den Eigenschaften eines evtl. aufmontierten Stents sowie dem Back-up des eingesetzten Führungskatheters und den Eigenschaften des eingesetzten Koronardrahtes ab. Extra Supportdrähte mit einem besonders harten Schaft strecken die Gefäße und können das Vorbringen eines Ballonsystems erleichtern, sind aber in Kombination mit einer weichen Drahtspitze schlechter zu steuern als sog. "Workhorse"-Drähte mit einer geringeren Schafthärte. Polymerbeschichtete Drähte passieren durch die Oberflächenbeschichtung ihrer Spitze zwar leichter stark gewundene Gefäße und enge Stenosen, bieten aber danach auch weniger Support für das Vorbringen eines Ballonsystems. In der Regel sind auf Ballonkathetern 2 Markierungen im Bereich der Ballonschultern aufgebracht. Bei sehr kleinen Ballons findet sich stattdessen ein Mittelmarker, weil der Marker das Crossing-Profil der Ballonspitze erhöhen würde.

Die reine Ballonangioplastie (,pure old balloon angioplasty" [POBA]) wurde inzwischen weitgehend durch die Ballondilatation mit Stentimplantation abgelöst, da eine systematische Stentimplantation zu besseren Akut- und Langzeitergebnissen führt (Abdeckung von Dissektionen, geringeres Recoil, Reduktion von Restenosen) [9]. Somit kommt den Ballonkathetern hauptsächlich eine Bedeutung in der Läsionsvorbereitung (Prädilation), bei der Implantation des Stents als Trägerballon und in der Optimierung der Stententfaltung und -apposition (Postdilatation) sowie bei der PTCA von In-Stent-Restenosen mittels DCB zu. Ein primäres Stenting kann zwar zu ähnlich guten Ergebnissen führen wie ein Stenting nach Prädilatation, bei der Behandlung von komplexen, insbesondere kalzifizierten Läsionen ist aber eine sorgfältige Läsionsvorbereitung zu empfehlen, um insbesondere eine Stentunterexpansi- on oder einen Stentverlust zu vermeiden [10-12].

Die POBA wird heute hauptsächlich in kleinen Gefäßen, zur Behandlung des Seitastes bei Bifurkationsstenosen (KissingBalloon-Technik u.a.) und zur Behandlung von In-Stent-Restenosen, hier häufig in Kombination mit medikamentenbeschichteten Ballons, eingesetzt.

Die verfügbaren Ballonsysteme sind entweder "semi-compliant" oder "noncompliant". "Semi-compliant" bedeutet, dass der angegebene Durchmesser bei Nominaldruck im Wasserbad erreicht wird, aber bei höheren Drucken der Ballondurchmesser noch deutlich weiter zunehmen kann; „non-compliant" bedeutet, dass diese Größenzunahme weniger als 10\% des Nominaldurchmessers beträgt. Die Compliance ist eine Funktion der Membranfestigkeit des Ballons, weshalb Semicompliant-Systeme in der Regel flexibler und leichter vorbringbar sind, während die steiferen Non-compliant-Systeme einen höheren "rated burst pressure" haben und deshalb für Hochdruckdilatationen geeignet sind. Durch die Verwendung von Doppelmembranen stehen inzwischen zusätzlich noch ausreichend flexible Ultrahochdruckballonsysteme zur Verfügung, die eine Druckapplikation von 35 bar und mehr erlauben.

Aus diesen Balloneigenschaften lassen sich grundsätzliche Empfehlungen zur Ballonnutzung ableiten. Bei der Wahl der Ballongröße ist einerseits zu beachten, dass nach dem Laplace-Gesetz die Wandspannung linear mit dem Durchmesser des Ballons ansteigt. Kleinere Ballons benötigen also höhere Druckwerte, um die gleiche Kraft auf die Gefäßwand auszuüben wie ein Ballon größeren Durchmessers. Andererseits muss ein „Überdehnen“ des Gefäßes mit nachfolgender Gefahr der Dissektion unbedingt vermieden werden, wenn ein umschriebenes, rigides Segment den Ballon an der Entfaltung hindert. Kann sich der Ballon in Teilen nicht vollständig entfalten, dann dehnt er sich an den Enden über seinen Nominaldurchmesser hinaus („Dogboning") - dieser Effekt ist bei Compliant- und Semi-compliant-Ballons besonders ausgeprägt. Aus diesem Grund ist die initiale Wahl eines kleinen, Non-compliant-Ballons bei Stenosen mit sehr rigiden Segmenten vorteilhaft: Der 
Tab. 1 Aktuell verfügbare Scoring-/Cutting-Ballons

\begin{tabular}{|l|l|l|l|l|}
\hline & $\begin{array}{l}\text { Wolverine } \\
\text { Cutting Balloon } \\
\text { Device }\end{array}$ & NSE alpha & Angiosculp & $\begin{array}{l}\text { Scoreflex } \\
\text { Scoring Balloon } \\
\text { Device }\end{array}$ \\
\hline $\begin{array}{l}\text { Katheterspitze } \\
\text { Eintrittsprofil }\end{array}$ & $0,017^{\prime \prime}(0,43 \mathrm{~mm})$ & $\begin{array}{l}0,016^{\prime \prime} \\
(0,401 \mathrm{~mm})\end{array}$ & $\begin{array}{l}0,025^{\prime \prime} \\
(0,635 \mathrm{~mm})\end{array}$ & $0,0223^{\prime \prime}(0,566 \mathrm{~mm})$ \\
\hline Crossing-Profil & $0,036^{\prime \prime}(0,91 \mathrm{~mm})$ & $\begin{array}{l}0,046^{\prime \prime} \\
(1,168 \mathrm{~mm})\end{array}$ & $\begin{array}{l}0,038^{\prime \prime} \\
(0,965 \mathrm{~mm})\end{array}$ & $0,0327^{\prime \prime}(0,830 \mathrm{~mm})$ \\
\hline
\end{tabular}

Ballon kann mit hohem Druck entfaltet werden, ohne das Gefäß außerhalb der Stenose zu verletzen.

Die meisten Stentfabrikate haben nur 2 oder 3 (selten 4) verschiedene Bautypen, die das gesamte Größenspektrum der Koronarien abdecken. Der angegebene Stentdurchmesser entspricht deshalb dem nominalen Durchmesser des Ballons, auf dem der Stent montiert ist. Gelingt bei Nominaldruck die Entfaltung des Stents in einer kalzifizierten Stenose nicht und muss deshalb der Druck erhöht werden, wird der Stent zwangsläufig in der nachgiebigeren Umgebung der Stenose überdilatiert. Deshalb ist in solchen Situationen eine ausreichende Läsionsvorbereitung von grundlegender Bedeutung. In der Regel sollte für die Vordilatation ein Ballondurchmesser von 0,5 mm unter der Referenzgröße des zu behandelnden Gefäßes gewählt werden und für die Nachdehnung dann ein adäquat dimensionierter NC-Ballon. Auch bei einer reinen Ballonangioplastie sollten keine überdimensionierten Ballons zum Einsatz kommen (maximales Verhältnis Ballon-/Gefäßdurchmesser von $1,1: 1)$.

\subsubsection{Medikamentenbeschichtete Ballons}

Medikamentenbeschichtete Ballons geben ihren antiproliferativen Wirkstoff (Paclitaxel und Sirolimus) aus einem Exzipient an die Gefäßwand ab. Damit sollen die Intima- bzw. Muskelzellproliferation als Mechanismen der Restenosierung unterdrückt werden. Aufgrund der sehr verschiedenen Beschichtungstechnologie der DCB ist nicht von einem Klasseneffekt auszugehen [13]. Einzelne DCB haben gute Ergebnisse in der Behandlung von Bare-Metal- und Drug-Eluting-In-StentRestenosen gezeigt [14-19].

Die Datenlage für die Behandlung von kleinen Gefäßen, Seitenästen bei Bifurkationsstenosen und langen Läsionen ist ge- ringer und weniger einheitlich [20-22]. Die aktuellen Leitlinien sehen eine Klasse-IAIndikation ausschließlich für die Behandlung von In-Stent-Restenosen [6, 20-22].

\subsubsection{Scoring- und Cutting-Ballons}

Das Prinzip dieser Devices ist die fokussierte Kraftübertragung des Ballons auf die Gefäßwand über Drahtsegmente zwischen Ballon und Gefäßwand, im Ballon integrierte Klingen oder Kunststofffäden (die bei der Balloninflation in die Gefäßwand gedrückt werden, aber im deflatierten Zustand eingefaltet sind) oder ScoringElemente (Nitinolkörbe, die den Ballon umschließen). Es gibt bisher keine ausreichende Evidenz, dass diese Devices grundsätzlich zu geringeren Restenoseraten führen als eine klassische Ballonprädilatation [23]. Sie können im Einzelfall aber eingesetzt werden, um harte Stenosen, die primär mit einem Hochdruckballon nicht dilatierbar sind, doch noch erfolgreich zu weiten. Ihr Einsatz in dieser Indikation wird aber durch ungünstigere Crossing-Profile und eine geringere Flexibilität limitiert (s. ๑ Tab. 1).

\subsection{Rotablation}

Die Rotablation wurde bereits in den 90erJahren in der Prä-DES-Stent-Ära entwickelt. Der ursprüngliche Gedanke war, durch die Abtragung von Plaquematerial die Restenosierungsrate im Vergleich zu einer reinen Ballonangioplastie zu senken. Dieses Konzept konnte aber niemals belegt werden. Gleichermaßen konnte nicht gezeigt werden, dass die grundsätzliche Läsionsvorbereitung kalzifizierter Stenosen mit einer Rotablation vor DESImplantation bessere Langzeitergebnisse als eine Läsionspräparation mit Ballonangioplastie und Rotablation nur als Bailout-Strategie (Notfallstrategie) erzielt [24].

Die hohe Erfolgsrate der Rotablation als Bail-out-Strategie für nichtdilatierbare
Stenosen hat aber dazu geführt, dass die Rotationsatherektomie anders als die direktionale Atherektomie immer noch ubiquitär eingesetzt und für diesen Zweck auch empfohlen wird (Ila, C) [6].

Das Prinzip der Rotablation ist die teilweise Plaqueabtragung durch eine druckluftgetriebene, schnell drehende Metallolive, die distal mit Diamantsplittern besetzt ist und auf einem speziellen Koronardraht vorgeschoben wird. Hartes Gewebe wird vom Bohrkopf erfasst und in Mikropartikel zerlegt, die so klein sind, dass sie die kardiale Mikrozirkulation passieren können; weiches Gewebe weicht dem Bohrkopf aus (Differential-Cutting). Der Einsatz des Rotablators zielt heute meist auf eine Plaquemodifikation ab, d.h. die Herstellung der Dilatierbarkeit einer harten Plaque. Hierzu wird ein im Verhältnis zur Gefäßgröße unterdimensionierter Bohrkopf eingesetzt, der neben der ablativen Wirkung auch zur Fissurbildung in den tieferen Kalkschichten führt.

Dies bedeutet, dass Bohrköpfe kleiner als das zu behandelnde Gefäß gewählt werden. Dementsprechend kann die Rotablation mit $6 \mathrm{~F}$ - (bis 1,5 mm-Bohrkopf) bzw. 7F- (bis 1,75 mm-Bohrkopf) Führungskathetern durchgeführt werden. Die Drahttechnik hat sich ebenfalls durch die Verwendung von Mikrokatheter bzw. OTW-Ballons wesentlich vereinfacht. Demzufolge wird der sog. Rotawire (Edelstahldraht als "floppy wire" oder "extra support wire ${ }^{\prime \prime}$ ) über diese nach Sondierung des Gefäßes mit gewöhnlichen "Workhorse"Führungsdrähten eingeführt. Nach Funktionsprüfung des Rotablators mittels DRAWSchema (D - "drops"; R - "rotation“ um 160.000-170.000 U/min; A - "advance“; W - „wire") sollte mit einem sog. PeckingManöver die Läsion bearbeitet werden. Hierbei sollte die Drehzahl nicht um mehr als $5000 \mathrm{U} / \mathrm{min}$ abfallen und die Behandlungszeit 15-20s nicht überschreiten. Das Legen eines passageren Schrittmachers wird in folgenden Fällen empfohlen: Hauptstamm, RCA, niedrige Ausgangsherzfrequenz (I, C) (•Abb. 1).

In jüngerer Zeit wurde die Rotationsatherektomie noch um das Verfahren der orbitalen Atherektomie ergänzt. Im Unterschied zur Rotablation ist die Bohrkrone am Ende der Antriebswelle exzentrisch montiert, sodass sie von der Zentrifugalkraft an 

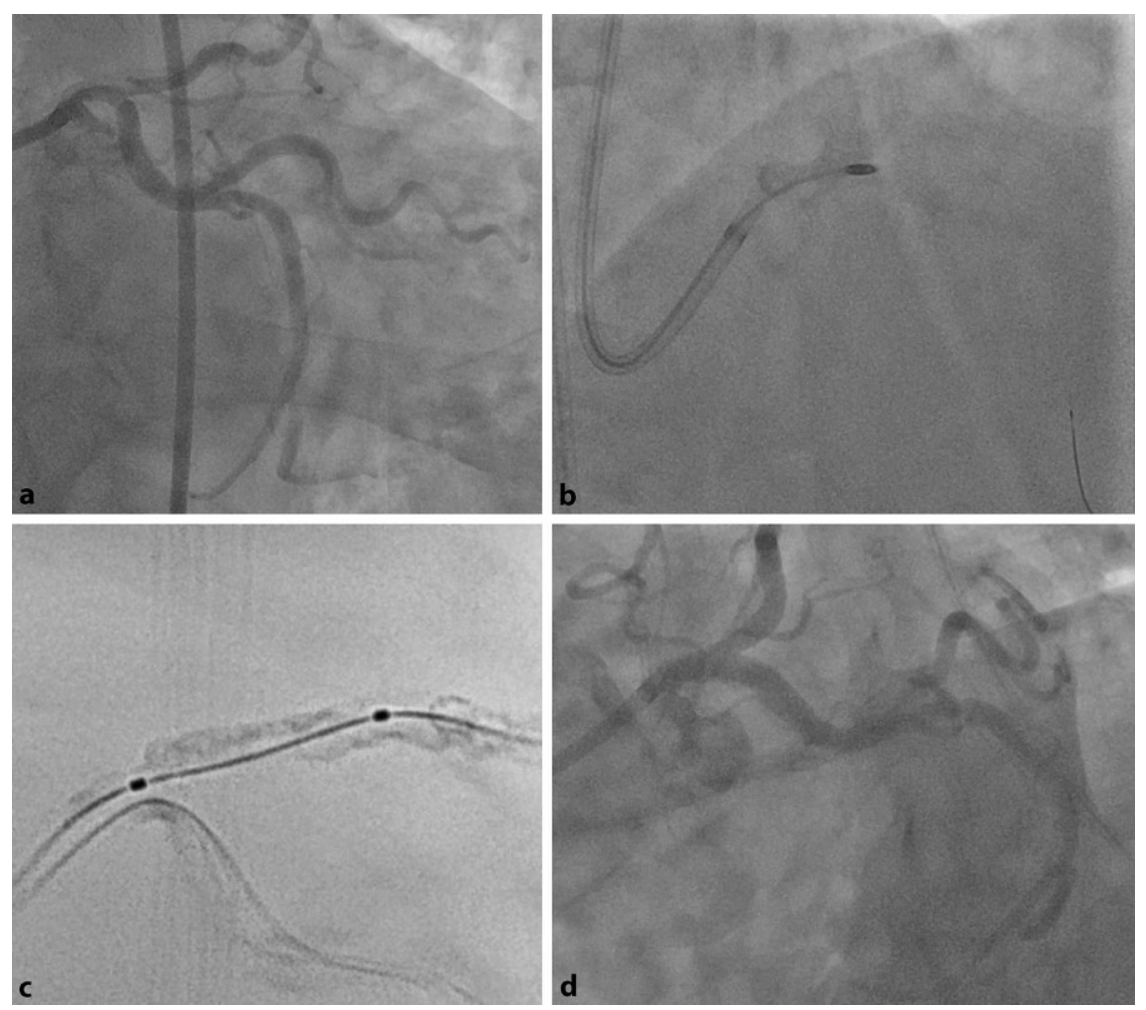

Abb. 1 \ Stark verkalkte Stenose des Hauptstamms (a). Rotablation mit einem Bohrkopfgröße $1,5 \mathrm{~mm}$ (b). Sichtbarer Nativkalk (c). Ergebnis nach Intervention (d)

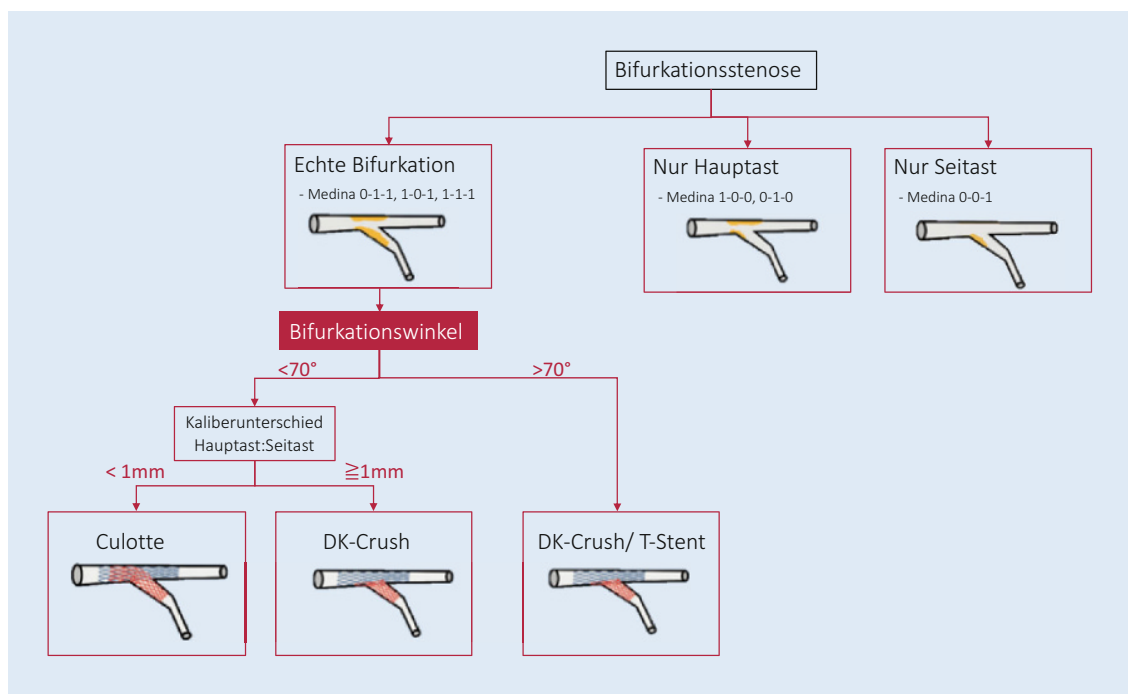

Abb. 2 ॥ Strategieauswahl bei betroffenem Haupt- und Seitast. (Mod. nach [30])

die Wand gedrückt wird. Deshalb kann dieselbe Krone abhängig von der Rotationsgeschwindigkeit das Gefäß auf verschiedene Durchmesser aufbohren. Außerdem ist grundsätzlich ein Blutfluss nach distal während der Atherektomie möglich.

\subsection{Lithotripsie}

Eine neuere Entwicklung zur Läsionspräparation kalzifizierter Läsionen ist die Lithotripsie. Hierzu wurden in einen Ballonkatheter mehrere Emitter integriert, die - bei einem mit niedrigem Druck (4atm) inflatierten Ballon - über die gesamte Zirkumferenz eine nicht fokussierte akusti- sche Pulswelle abgeben. Ähnlich wie bei der Lithotripsie kann die Schallwelle beim Auftreffen auf oberflächliche oder tiefe Verkalkungen der Gefäßwand hohe Energien übertragen, die den Kalk zertrümmern. Die Effektivität und Sicherheit des Systems wurden bisher in einer peripheren und einer koronaren Studie belegt $[25$, 26]. Weitere randomisierte Studien müssen den genauen Stellenwert der Methode noch untersuchen. Gegenwärtig bietet sich die Methode insbesondere für die Behandlung nichtdilatierbarer Stenosen ggf. auch in Kombination mit einer Rotationsatherektomie an. Fallberichte zeigen auch die Machbarkeit einer Behandlung von unterexpandierten Stents (aktuell noch „offlabel") [27].

\subsection{Praktisches Vorgehen}

bei speziellen anatomischen Situationen

\subsubsection{Bifurkationsstenosen}

Grundsätzlich sollte vor der Planung einer Bifurkationsstenosenintervention eine Einteilung der Stenose entsprechend der Medina-Klassifikation erfolgen (s. Zusatzmaterial Online, zusätzliche Abb. 30) [28]. Hiernach orientiert sich das weitere interventionelle Vorgehen mit der Entscheidung, ob ein "Provisional" -Stenting-Ansatz oder a priori eine 2-Stent-Strategie gewählt wird. Entsprechend den allgemeingültigen Empfehlungen ist dabei auf den Abgangswinkel des Seitastes zu achten, da bei Abgangswinkeln $<70^{\circ}$ spezielle Bifurkationstechniken eingesetzt werden müssen. Ein ebenfalls wichtiges Kriterium ist die Beachtung der natürlichen Verjüngung der Gefäßdiameter nach distal. Dementsprechend besteht eine konstante Relation zwischen distalem und proximalem Hauptast sowie dem Seitast, welche im Gesetz von Finet mathematischen Ausdruck $(D 1=(D 2+D 3) * 0,678)$ findet [29]. Für die Stentgrößenauswahl ist der distale Gefäßdiameter entscheidend.

Für den klinischen Alltag gilt es, einen der Stenosemorphologie und der Gefäßanatomie entsprechenden "Workflow" zu implementieren. Hierbei ist nach Bestimmung der Medina-Klassifikation die Beurteilung des Bifurkationswinkels entscheidend. Anschließend erfolgt die Abschätzung der Gefäßdiameter. Bei einer 


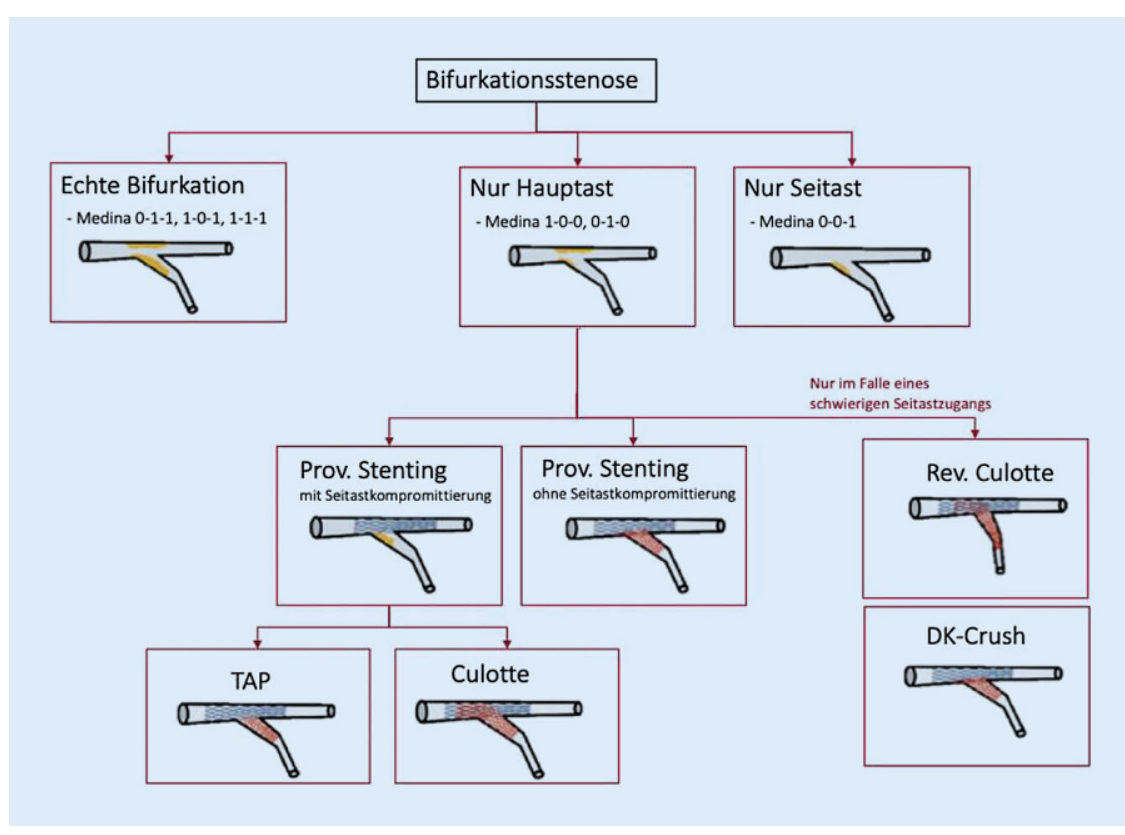

Abb. 3 ॥ Strategieauswahl bei isolierter Hauptastbeteiligung. (Mod. nach [30])

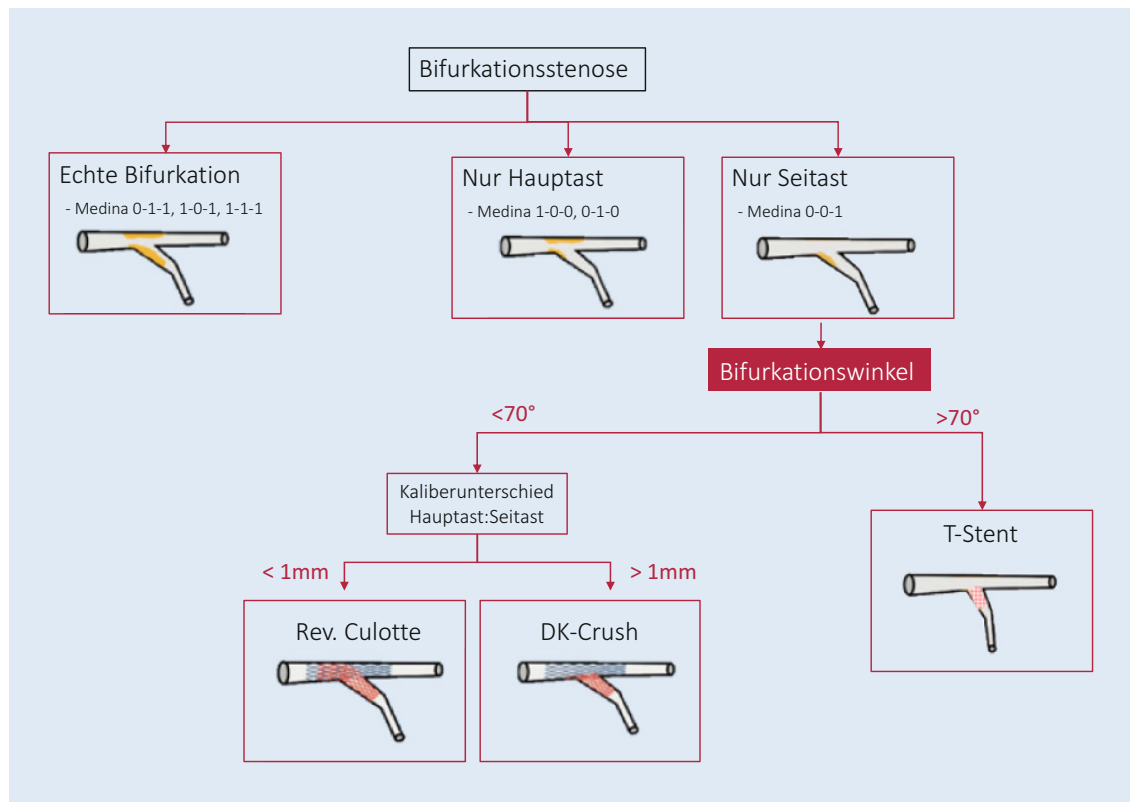

Abb. 4 ॥ Strategieauswahl bei isolierter Seitastbeteiligung. (Mod. nach [30])

Differenz von mehr als $1 \mathrm{~mm}$ zwischen Hauptast und Seitast erscheint für alle Bifurkationsmorphologien die Culotte-Technik weniger empfehlenswert. Für echte (Seit- und Hauptast betroffen) Bifurkationsstenosen mit einem Abgangswinkel $<70^{\circ}$ und einem Kaliberunterschied zwischen Hauptast und Seitast $>1 \mathrm{~mm}$ ist eine DK-Crush-Technik oder eine TAPTechnik ideal (s. - Abb. 2).

Nach wie vor gilt, dass ein sog. „provisional stenting" die beste Methode ist, um einen alleinig erkrankten Hauptast zu behandeln (I, A) [6]. Sofern nach Stentimplantation im Hauptast der Seitast kompromittiert ist, kann nachfolgend zunächst eine proximale Optimierungstechnik (POT) erfolgen (s. - Abb. 3). Sollte dies nicht zu einem adäquaten Ergebnis im Seitast führen, kann die „Kissing-ballon"-Technik angewandt werden. Ist hiernach der Seitast weiterhin hochgradig stenosiert, sollte mit einer TAP-Technik oder einer Culotte-Technik abschließend behandelt werden.
Grundsätzlich ist bei allen Bifurkationstechniken darauf zu achten, dass nach „Kissing-ballon"-Manöver der proximaleStentanteil im Hauptast mit einem sog. „FinalPOT" versorgt wird [28].

Liegt lediglich eine Seitaststenose vor, sollte eine genaue Evaluierung ggf. mit intravaskulärer Bildgebung des Bifurkationsbereichs erfolgen. Ist der Hauptast nicht stenosiert, kann eine T-Technik angewandt werden. Allerdings liegt in den meisten Fällen eine arteriosklerotische Beteiligung des Hauptastes vor, weshalb eine 2-Stent-Strategie (z. B. Reverse-Culotte oder DK-Crush-Methode) zum Einsatz kommen kann (s. - Abb. 4). Allerdings ist diese Konstellation immer individuell und unter Berücksichtigung aller infrage kommender Techniken zu entscheiden.

\subsubsection{Chronische Koronarverschlüsse} Aktuelle Richtlinien empfehlen (Ila, B), dass die perkutane Revaskularisierung von chronischen Gefäßverschlüssen („chronic total occlusion" [CTO]) bei Patienten mit medikamentenresistenter Angina pectoris oder mit einem großen Versorgungsgebiet und entsprechend dokumentierter Ischämie in Betracht gezogen werden sollte [31].

Ein Schlüssel zum Erfolg einer Rekanalisation einer CTO ist die gute Vorbereitung. Hierzu sollte neben einem entsprechenden bildgebenden Nachweis der Vitalität (MRT) des entsprechenden Versorgungsgebietes der J-CTO-Score bei antegradem Vorgehen Anwendung finden (s. A Abb. 5).

Er beschreibt unabhängige Prädiktoren, die die antegrade Rekanalisationswahrscheinlichkeit und die Schnelligkeit der antegraden Drahtpassage reflektieren (jeweils mit 1 Punkt). Insbesondere beinhaltet er einen vorangegangenen gescheiterten Versuch, eine CTO wiederzueröffnen, den angiographischen Nachweis einer starken Verkalkung, einen Verlaufswinkel des Gefäßes $\geq 45^{\circ}$ innerhalb des okkludierten Segments, einen „blunt proximal stump" sowie eine Okklusionslänge $>20 \mathrm{~mm}$. Dementsprechend werden CTOLäsionen als leicht, mittelschwer, schwer und sehr schwer (J-CTO-Scores von 0, 1, 2 bzw. $\geq 3$ ) klassifiziert [32]. Während erfahrene Untersucher auch sehr komplexe Prozeduren erfolgreich behandeln können, sollten diejenigen, die am Beginn ihrer 


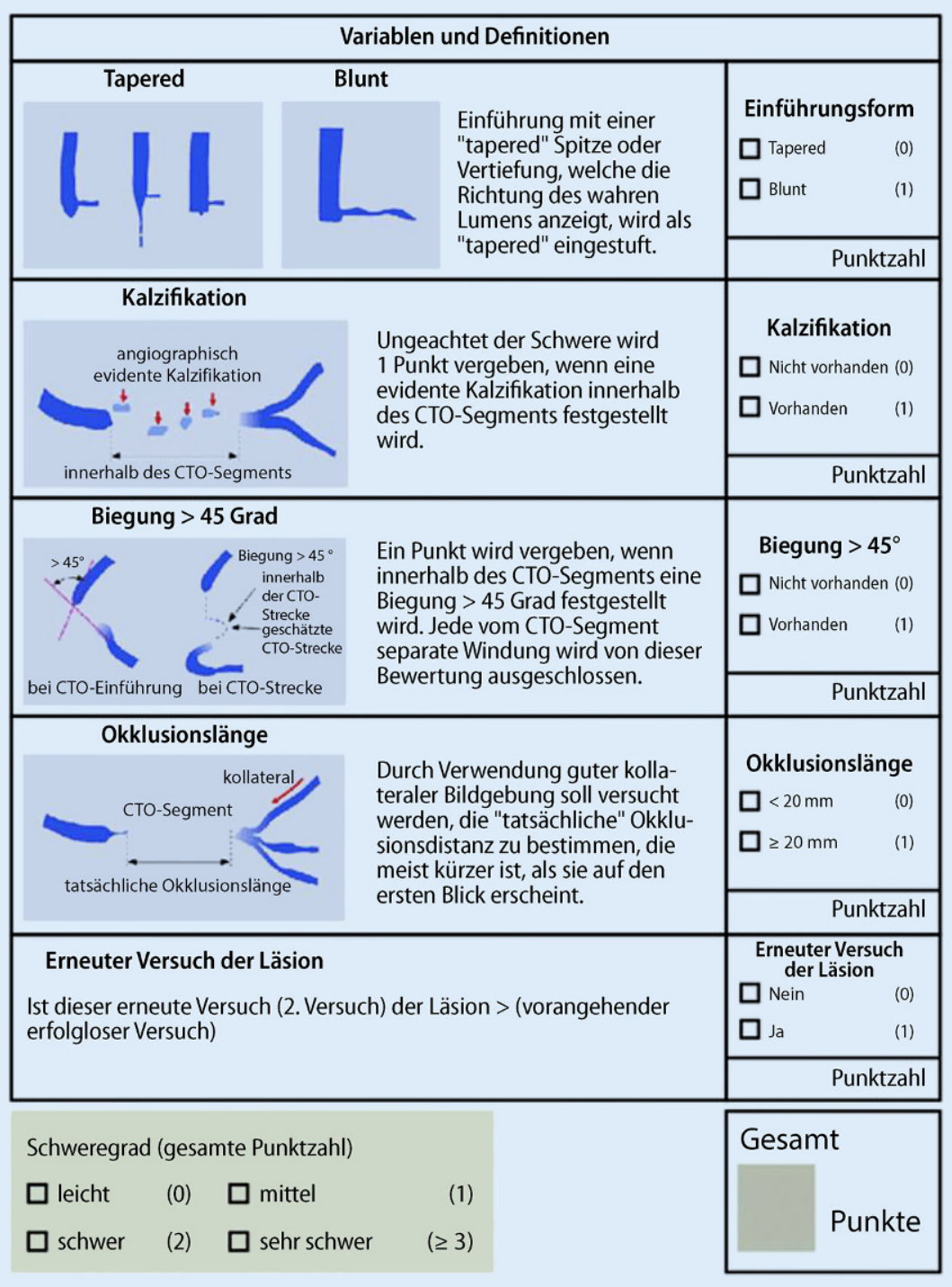

Abb. 5 ^ J-CTO-Score. (Mod. nach [32])

Lernkurve stehen, eher „einfachere" Fälle auswählen (J-CTO-Punktzahl 0 oder 1). Antizipierbar schwierigere Prozeduren (J-CTO 22) sollten an CTO-Zentren oder mithilfe eines Proctors durchgeführt werden $(I, C)$.

Neben einer guten Vorbereitung einer Rekanalisationsmaßnahme ist die gute $\mathrm{Vi}$ sualisierung einer CTO eine wichtige Voraussetzung für eine erfolgreiche Intervention. Hierzu dient die simultane kontralaterale Injektion (I, C), die wertvolle Informationen über die Morphologie, Kollateralisierung, Länge des Verschlusses und Komplexität der CTO liefern kann. Eine CTO-PCI ohne duale Injektion wird nur bei unzureichender kontralateraler Kontrastierung oder adäquater ipsilateraler Versorgung empfohlen (I, C). Mögliche Zugangswege für die Katheter können bifemoral, radial/ femoral oder unifemoral sein. Hinsichtlich der Größe des Führungskatheters werden in den meisten Fällen bei antegrader Rekanalisationstechnik ein 7 F-Katheter zur Rekanalisation und 5-6 F für die Kontrastierung über Kollateralen empfohlen. Bei retrograder Rekanalisationstechnik empfiehlt sich meist die Verwendung von zwei 7 F-Führungskathetern.

Für Rekanalisationen im LCA-Stromgebieterscheint ein Extra-Back-up-Führungskatheter am geeignetsten. Für das RCAStromgebiet hat sich ein AL 0,75 bis 1 bewährt. Trotzdem gibt es bestimmte Anatomien, bei denen andere Führungskathe- terkonfigurationen sinnvoll sind (z.B. JR oder IMA-Katheter bei ostialen Verschlüssen). Der Untersucher sollte mit den Techniken der tiefen Intubation, Anker-Ballon oder der Verwendung von "Mother-andChild"-Kathetern vertraut sein.

Die Führungsdrahttechnologie hat sich in den letzten Jahren rasant entwickelt und hat zur Entwicklung verschiedenster CTO-PCI-Techniken entscheidend beigetragen. Grundsätzlich gilt, dass kein einzelner Draht für alle Läsionsmorphologien geeignet ist. Gleichfalls entscheidend für den Erfolg einer СТО sind die Mikrokatheter, da sie eine bessere Drahtführung ermöglichen als auch einen wiederholten Wechsel der Drähte zulassen (s. - Tab. 2; - Abb. 6).

Der antegrade Rekanalisationsversuch ist der Eckpfeiler der CTO-PCl und wird in etwa $75 \%$ der Fälle erfolgreich angewandt [34]. Die damit verbundene Drahteskalation ist das aktuell empfohlene Vorgehen bei wenig komplexer CTO mit guter distaler Landungszone und einer Läsionslänge $<20 \mathrm{~mm}$. Der Strategiealgorithmus wurde unlängst vom EuroCTO Club publiziert [31].

Ein "True-to-true"-Lumen-Vorgehen ist die derzeitig präferierte Vorgehensweise. Dennoch gibt es Situationen, in denen ein distales Dissektion/Rentry-Manöver angewandt werden muss. Dies trifft v. a. für komplexe CTO-Morphologien zu. Derzeitig zu Beginn empfohlene Führungsdrähte der ersten Wahl sind meist getapert (spitz zulaufend) und polymerbeschichtet, mit relativ niedriger "tip-load" (Fielder XT, $X T-R, X T-A)$. Einzig nicht getaperter Draht für den Primäreinsatz ist der Ultimate $3 \mathrm{~g}$. Sollte die proximale Verschlusskappe nicht penetriert werden können, muss ein härterer getaperter Draht zur Punktion gewählt werden (z. B. Confianza Pro, Hornet 14). Danach sollte als "Step-down"-Strategie wieder auf einen weicheren Draht gewechselt werden. In der CTO-Läsion kann im Stepup- oder Step-down-Modus auf sehr gut steuerbare getaperte intermediäre Drähte aus der Gaia-Familie (z. B. Gaia II oder III) gewechselt werden. Ziel ist die intraluminale Rekanalisation.

Sollte es zu einer subintimalen Situation im Bereich der distalen Landezone kommen (bestätigt durch die kontralaterale Injektion), steht eine Reihe an Techniken 
Tab. 2 Zurzeit verfügbare Mikrokatheter bei CTO-Intervention

\begin{tabular}{|c|c|c|c|}
\hline Hersteller & Katheter & Länge & $\begin{array}{l}\text { Distaler Schaft oder } \\
\text { äußerer Diameter }\end{array}$ \\
\hline \multirow[t]{3}{*}{ Asahi Intecc } & Tornus & $135 \mathrm{~cm}$ & 2,1 und $2,6 \mathrm{Fr}$ \\
\hline & Corsair und Corsair Pro & $135 \mathrm{~cm}, 150 \mathrm{~cm}$ & $2,6 \mathrm{Fr}$ \\
\hline & Caravel & $135 \mathrm{~cm}, 150 \mathrm{~cm}$ & $1,9 \mathrm{Fr}$ \\
\hline $\begin{array}{l}\text { Boston } \\
\text { Scientific }\end{array}$ & Renegade $^{\mathrm{TM}} 18$ & $105 \mathrm{~cm}, 115 \mathrm{~cm}, 135 \mathrm{~cm}$ & $2,5 \mathrm{Fr}$ \\
\hline IMDS & NHancer Pro X & $135 \mathrm{~cm}, 155 \mathrm{~cm}$ & $2,3 \mathrm{Fr}$ \\
\hline $\begin{array}{l}\text { Roxwood } \\
\text { (now BTG) }\end{array}$ & $\begin{array}{l}\text { MicroCross } 14^{\circledR} \text { und } \\
\text { MicroCross 14ES }\end{array}$ & $155 \mathrm{~cm}$ & $1,6 \mathrm{Fr}$ \\
\hline Terumo & Finecross $^{\circledR}$ & $130 \mathrm{~cm}, 150 \mathrm{~cm}$ & $1,8 \mathrm{Fr}$ \\
\hline \multirow[t]{6}{*}{ Teleflex } & Venture $^{\circledR}$ & $\begin{array}{l}145 \mathrm{~cm} \text { („rapid exchange“) } \\
140 \mathrm{~cm} \text { („over-the-wire“) }\end{array}$ & $2,2 \mathrm{Fr}$ \\
\hline & SuperCross ${ }^{\mathrm{TM}}$ & $\begin{array}{l}130 \mathrm{~cm}, 150 \mathrm{~cm} \\
\text { Spitzenwinkel }\left(45^{\circ}, 90^{\circ} \text { oder }\right. \\
\left.120^{\circ}\right)\end{array}$ & $2,1 \mathrm{Fr}$ \\
\hline & Turnpike ${ }^{\circledR}$ & $135 \mathrm{~cm}, 150 \mathrm{~cm}$ & $2,6 \mathrm{Fr}$ \\
\hline & Turnpike ${ }^{\circledR}$ LP & $135 \mathrm{~cm}, 150 \mathrm{~cm}$ & $2,2 \mathrm{Fr}$ \\
\hline & Turnpike ${ }^{\circledR}$ Spiral & $135 \mathrm{~cm}, 150 \mathrm{~cm}$ & $3,1 \mathrm{Fr}$ \\
\hline & Turnpike ${ }^{\circledR}$ Gold & $135 \mathrm{~cm}$ & $3,2 \mathrm{Fr}$ \\
\hline Acrostak & M-CATH & $135 \mathrm{~cm}$ & $2,25 \mathrm{Fr}$ \\
\hline $\begin{array}{l}\text { Merit Medi- } \\
\text { cal }\end{array}$ & SwiftNINJA & $135 \mathrm{~cm}$ & $2,4 \mathrm{Fr}$ \\
\hline
\end{tabular}

zur Verfügung, um das wahre Lumen zu erreichen. Hierzu kann man Drahttechniken (Parallel-Wire-Technik, Mini-STAR) als auch Device-basierte Ansätze verwenden (Stingray). Bei der Paralleldrahttechnik ist oft die Verwendung eines Dual-LumenMikrokatheters von Nutzen.

Retrograde Techniken sind bei fehlendem antegradem Erfolg und adäquaten Kollateralen zu erwägen. Weitere Entscheidungskriterien für den retrograden $\mathrm{Zu}$ gang können eine stark veränderte Landungszone oder eine Bifurkation im Bereich der distalen Kappe sein. Hierzu sollten entsprechende Mikrokatheter (Corsair, Caravel, Turnpike) bzw. Drähte (SUOH 03, Sion Black) Verwendung finden. Septale Kollateralen sind immer zu bevorzugen, da die Passage der epikardialen Kollateralen mit einem höheren Risiko einhergehen und dementsprechend den erfahrenen Untersuchern überlassen werden sollten. Ebenfalls ist bei der retrograden Rekanalisation ein Crossing des Führungsdrahtes im wahren Lumen anzustreben. Sollte dies nicht möglich sein, kann zum Teil die "kissing-wire"-Technik angewandt werden. Darüber hinaus ist die ReverseCART-Technik (mit oder ohne Knuckle-wire-Technik) und anschliessender Externa- lisation eines entsprechenden Führungsdrahtes (RG3) sicher und effektiv anwendbar. Weitere Techniken, wie das Tip-in-Manöver, können bei der retrograden Rekanalisation zum Einsatz kommen.

Entsprechend den Leitlinien konnte zuletzt das Konzept des sog. „Hybrid-Algorithmus" die Erfolgsrate weiter verbessern. Ein modifizierter und aktualisierter Algorithmus des EuroCTO Clubs ist dem entsprechenden Positionspapier zu entnehmen [31].

\subsubsection{In-Stent-Restenosen}

Die alleinige Ballondilatation von In-StentRestenosen (ISR) erzielt gute Akutergebnisse, allerdings ist sie mit einer hohen Rezidivrate assoziiert, weshalb alternative Verfahren angewandt werden sollten (III, C). Die Behandlung mit einem Cutting- oder Scoring-Ballon führte in einer randomisierten Untersuchung zu keiner signifikanten Reduktion der Restenose [35]. Allerdings war aber das "Verrutschen“ der Ballons seltener zu beobachten. Auch die Rotablation war in RCTs der alleinigen Ballonangioplastie nicht überlegen. Dementsprechend ist die Rotablation lediglich als Möglichkeit anzusehen, um ggf. eine ISR bei massiver Unterexpansion besser vor- zubehandeln (IIb, C). Die Anwendung der Rotablation ist im Stent nicht immer ungefährlich und sollte deshalb mit großer Zurückhaltung durchgeführt werden und dem erfahrenen Untersucher überlassen bleiben (I, C).

Hinsichtlich der Versorgung von ISR mit DES oder DCB ergeben sich unterschiedliche Aspekte, die v. a. die Morphologie der ISR berücksichtigen sollten. In großen Metaanalysen zeigten sich keine signifikanten Unterschiede zwischen DES und DCB in Bezug auf klinische Endpunkte [36].

Im Hinblick auf den angiographischen Endpunkt des sog. „late lumen loss" scheint der DCB dem DES tendenziell überlegen zu sein. Trotzdem ist die Neoatherosklerose möglicherweise besser mit einem DES und eine reine Intimahyperproliferation besser mit einem DCB behandelt [19].

\subsubsection{Bypassgefäße}

Stenosen in SVG auf RCA können meist mit MP-Führungskathetern mit sehr gutem Back-up versorgt werden. Für SVG auf LCA sind JR- oder AL-Führungskatheter zu empfehlen. Abhängig von der Anatomie können natürlich auch alternativ konfigurierte Führungskatheter (z. B. LCB, RCB) Verwendung finden.

Bei der PCl von SVG gibt es Hinweise, dass Patienten, die einem Direktstenting unterzogen wurden, eine weniger hohe CK bzw. CK-MB-Erhöhung aufwiesen als Patienten mit vorheriger Dilatation [37]. Allerdings gibt es auch retrospektive Studien, die keine Nachteile einer vorherigen Dilatation hinsichtlich klinischer Parameter belegen konnten [38]. Derzeit kann deshalbhinsichtlich einer Prädilatation keine eindeutige Empfehlung ausgesprochen werden.

Obwohl in den europäischen als auch amerikanischen Leitlinien keine eindeutige Empfehlung hinsichtlich der Verwendung von DES ausgesprochen wird, empfiehlt sich trotz uneinheitlicher Datenlage die Verwendung von DES (Ila, C). Bioresorbierbare Scaffolds können bei Bypassstenosen aktuell nicht empfohlen werden (III, C).

Die PCl von SVG-Stenosen wird als Intervention mit hohem Risiko angesehen, da das Atherofibrom für distale Embolisation anfälliger ist. Es wurden verschiedene Ansätze evaluiert, um die Emboli- 


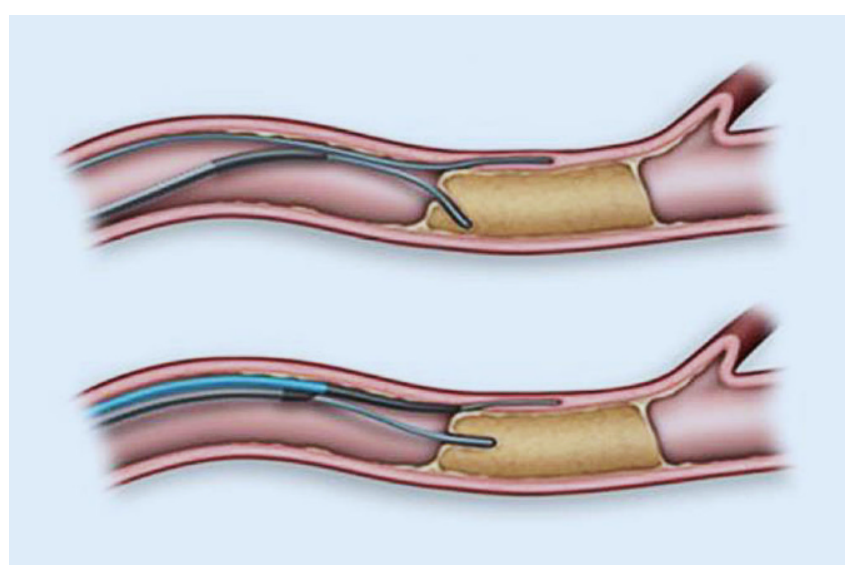

Abb. $6 \triangleleft$ Parallelwire-Technik. (Mod. nach [33])
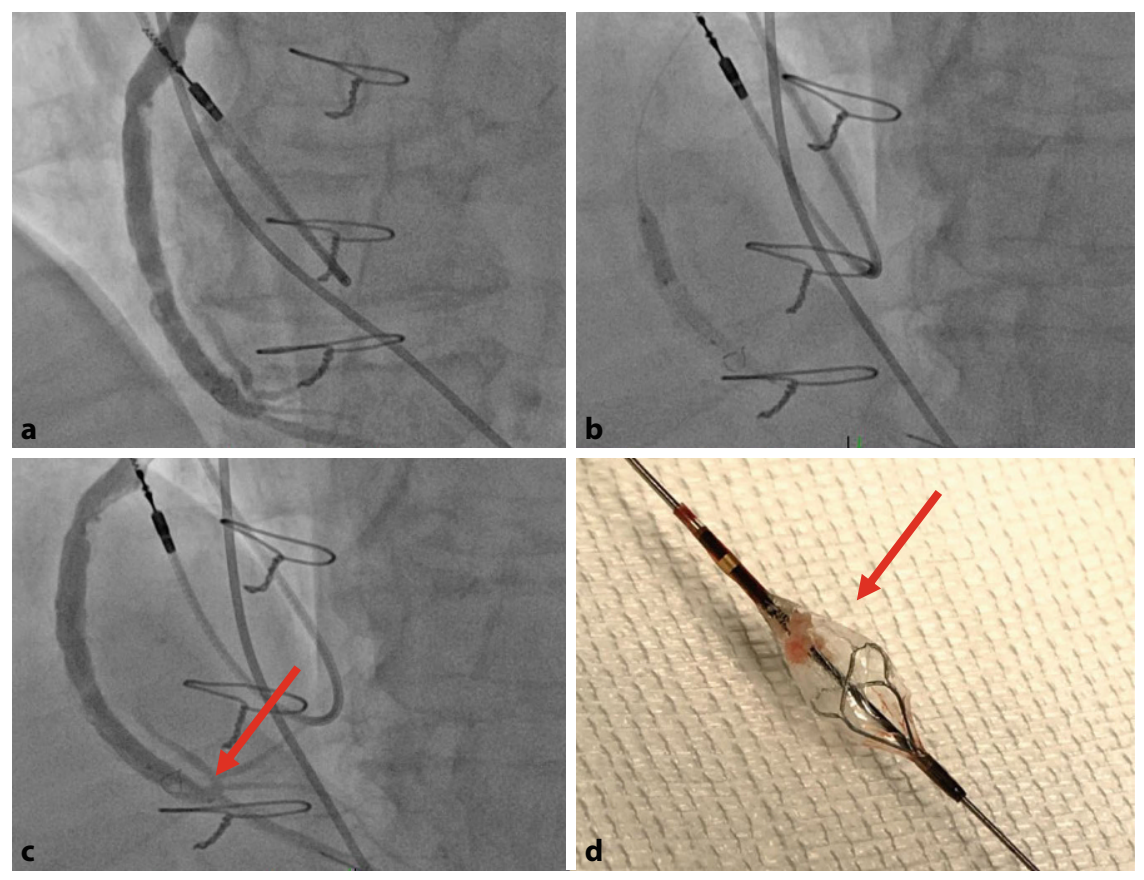

Abb. $7 \Delta$ a Stenose eines Venenbypasses. b PClunter Verwendung eines Embolieprotektionssystems. c, $\mathbf{d}$ Radiologisch und makroskopisch sichtbarerThrombus im Filterdes Protektionssystems (roter Pfeil)

sationen und damit Slow-Flow- bzw. NoFlow-Situation zu verhindern. Distale Protektionssysteme mit Filtern haben in einer randomisierten Studie die insgesamt besten Ergebnisse gezeigt. Obwohl eine einzelne randomisierte Studie die Verwendung unterstützt, sind allerdings die Daten aus Beobachtungsstudien und aus großen Registern widersprüchlich [39-41]. Prinzipiell gilt, dass - sofern eine Anwendung sinnvoll ist und ein entsprechend großes Versorgungsgebiet gefährdet erscheint die Verwendung von distalen Protektionssystemen erwogen werden sollte (Ila, B) (s. ๑ Abb. 7).

\subsubsection{Stenosen des ungeschützten Hauptstammes}

Die Hauptstammstenose stellt eine schwierig zu behandelnde Bifurkationsstenose dar. Bei der Planung und Durchführung der Hauptstamm-PCl wird der Einsatz der intravaskulären Bildgebung empfohlen (Ila, B). Bei Interventionen des Hauptstamms sollten ausschließlich DES zur Anwendung kommen, wobei je nach Stenosemorphologie eine 1- bzw. 2-StentStrategie infrage kommt. Zeigt sich nur „provisional stenting" die bevorzugte Methode (I, A). Bei der Wahl der richtigen 2-Stent-Strategie sind die anatomischen Bedingungen (Bifurkationswinkel, Gefäßein Tochtergefäß betroffen, ist das sog. diameter) zu berücksichtigen ( $\mathbf{A}$ Abb. 8). Gemäß Datenlage ist derzeit die DKCrush-Technik den anderen Strategien (TAP, Culotte, Mini-Crush) vorzuziehen (Illb, B).

\subsubsection{Ostiale Stenosen}

Die $\mathrm{PCl}$ von ostialen Läsionen in Koronararterien oder aortokoronaren Bypass-Grafts können eine besondere Herausforderung darstellen und sind mit höheren prozeduralen und mittelfristigen Komplikationsraten verbunden. Wichtig ist, dass gerade bei aortoostialen Läsionen ein potenzieller Vasospasmus ausgeschlossen ist, der durch Führungskatheter verursacht sein kann. Die Verwendung von intravaskulärer Bildgebung (OCT oder IVUS) sollte insbesondere bei ostialen Läsionen des Hauptstamms erwogen werden (Ila, B). Dabei können die Beurteilung der Plaqueverteilung als auch die Bestimmung der Läsionslänge und des Gefäßdiameters von großer Bedeutung sein.

Die Läsionspräparation mit DebulkingDevices sollte durchgeführt werden, wenn es zu einer unzureichenden Entfaltung der Ballonsysteme im Rahmen der Vordilatation kommt. Die Durchführung der Rotablation oder auch neuerdings einer Lithotripsie können Alternativen hierzu darstellen, ohne dass hierzu ausreichende Evidenz der Überlegenheit gegenüber der Ballonangioplastie vorhanden ist.

Die Stentpositionierung birgt einige Herausforderungen, weshalb v. a. die exakte angiographische Projektion wichtig ist (s. - Tab. 3). Weiterhin ist die richtige Technik des Führungskathetermanagements bei aortoostialen Läsionen von essenzieller Bedeutung. Hier kann auch die Verwendung eines zusätzlichen koronaren Führungsdrahtes (Markerdraht) in der Aorta ascendens die exakte Positionierung des Stents erleichtern. In Einzelfällen kann die Verwendung von spezifischen Stentsystemen (Ostial Pro) erwogen werden.

Für ostiale Läsionen der LAD oder des RCX kann die sog. "Draw-back“Technik die möglichst genaue Stentpositionierung ermöglichen. Hierbei wird ein zweiter Führungsdraht in das nichtbetroffene Gefäß positioniert. Ein leicht unterdimensionierter Ballon wird dann in das Hauptgefäß gelegt und insuffliert. 


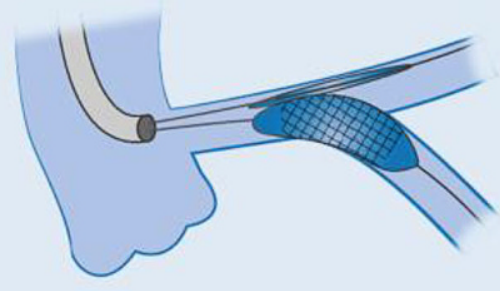

a

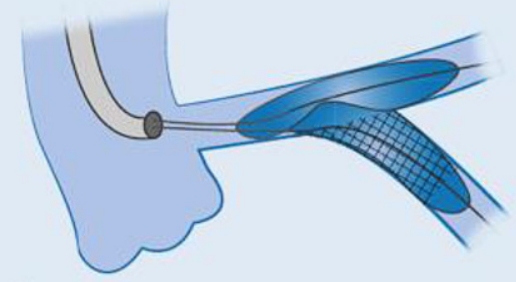

d

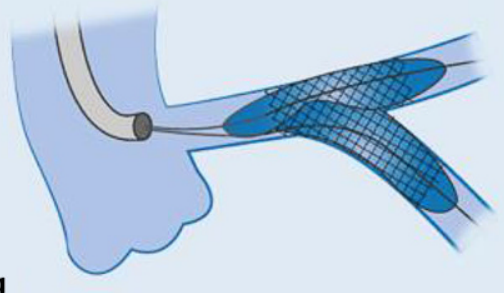

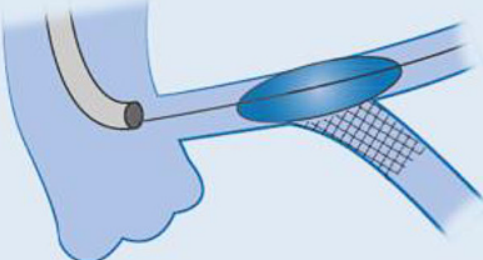

b

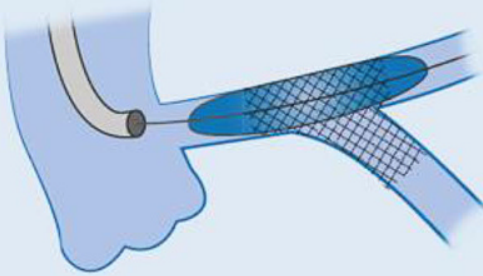

e

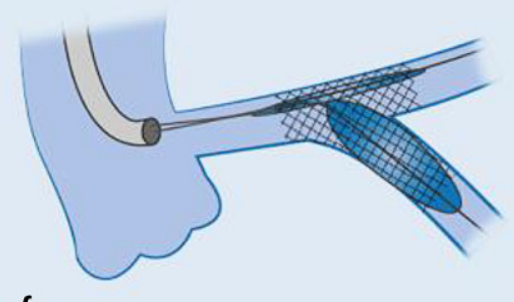

f

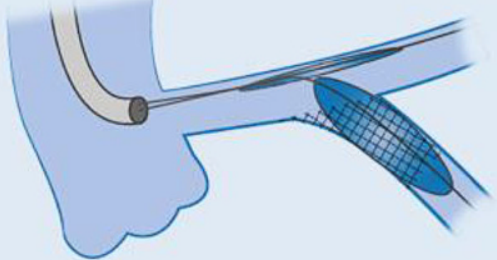

c

c

Abb. $8 \Delta$ DK( ,double kiss")-Crush-Technik: Drahteinlage in den Haupt- und Seitast, Vorbringen eines Stents in den Seit- und eines Ballons in den Hauptast. a Implantation des Stents in den Seitast; $\mathbf{b}$ Entfernen von Stentballon und Koronardraht aus dem Seitast, Ballondilatation im Hauptast mit "crush" des Seitaststents; cerneute Drahtsondierung des Seitasts durch die Stentstreben hindurch und Eröffnen der Stentstreben im Bereich des Seitastostiums; $\mathbf{d}$ erstes Kissing-Ballon-Manöver; e Implantation des zweiten Stents in den Hauptast; $\mathbf{f}$ Nachdilatation im Seitast; $\mathbf{g}$ zweites Kissing-Ballon-Manöver; abschließende proximale Optimierungstechnik (POT) nicht abgebildet. (Adaptiert nach [42]; mit freundlicher Genehmigung von $\odot$ John Wiley \& Sons, Inc. All Rights Reserved)

Der nicht abgesetzte Stent wird dann gegen den im Hauptast inflatierten Ballon zurückgezogen (s. - Abb. 9 und 10).

\subsubsection{Kalzifizierte Stenosen}

Stark kalzifizierte Stenosen stellen immer noch eine große Herausforderung in der Behandlung dar. Im Vorfeld der Behandlung kann die Evaluierung der Stenose mittels intravaskulärer Bildgebung von Vorteil sein (IIb, C). Insbesondere das Ausmaß der Verkalkung kann wesentlichen Einfluss auf die Strategieentscheidung nehmen. Ab einer Zirkumferenz von mehr als $270^{\circ}$ ist eine alleinige Ballondilatation wahrscheinlich nur ungenügend, und weitere Debulking-Verfahren (z. B. Rotablation, orbitale Atherektomie, Lithoplastie) sollten erwogen werden (Ila, C) (s. - Abb. 11).
Für einen optimales Back-up gilt grundsätzlich die adäquate Auswahl des Führungskatheters, ggf. die Verwendung von sog. Extension-Kathetern (z. B. Guidezilla, Guideliner, s. - Abb. 12). Ebenfalls ist zur Verbesserung des Back-ups in manchen Fällen die sog. Anker-Ballon-Technik möglich, setzt aber eine entsprechende Anatomie voraus (s. D Abb. 13).

Ein entscheidender Faktor bei komplex verkalkten Läsionen kann unter Umständen die Verwendung eines Mikrokatheters sein, der aufgrund seines CrossingProfils oftmals eher eine Stenose überwinden kann als ein Ballon. Dementsprechend kann in vielen Fällen der Einsatz eines Mikrokatheters erwogen werden (Ilb, C).

Für die Vordilatation sind bei kalzifizierten Läsionen Non-compliant-Ballons empfohlen (I, C). Aufgrund ihrer relativ ge- ringen Diameterzunahme in Abhängigkeit der Druckwerte wird insbesondere bei hohen Druckwerten (atm) eine Überexpansion außerhalb der Läsion vermieden. Insbesondere zur Vermeidung von Dissektionen im Stentkantenbereich ist der Einsatz eines NC-Ballons empfehlenswert (I, C).

Besondere Erwähnung sollte der Ultrahochdruckballon (OPN-Ballon, SIS Medical, Switzerland) finden. Dieser aus 2 Lagen produzierte Ballon wurde für die Dilatation mit Drücken bis 35 atü und darüber entwickelt. Limitation ist sein deutlich erhöhtes Crossing-Profil, weshalb er in höhergradigen Stenosen häufiger nicht eingebracht werden kann.

Dem Einsatz von Scoring-/Cutting-Ballons kommt gerade in der kalzifizierten Läsion eine besondere Bedeutung zu. Oftmals vereinfachen sich hierdurch die Posi- 
Tab. 3 Wichtige Projektionen zur Beurteilung von ostialen Läsionen

\begin{tabular}{|l|l|}
\hline $\begin{array}{l}\text { Gefäß mit } \\
\text { ostialer Läsion }\end{array}$ & Empfohlene Aufnahme \\
\hline LM & AP kranial oder LAO kranial \\
\hline RCA & LAO oder besser LAO kaudal, um die Ebene des Ostiums darzustellen \\
\hline LAD & $\begin{array}{l}\text { Kombination von kaudalen Darstellungen. LAO kaudal zeigt typischerweise } \\
\text { weniger Überschneidungen mit Rami oder dem RCX, stellt ihn aber kürzer } \\
\text { dar als die RAO kaudale Darstellung }\end{array}$ \\
\hline RCX & LAO kaudal, im Fall einer Überlappung kann die LAO kranial helfen \\
\hline Bypassgraft & $\begin{array}{l}\text { Normalerweise LAO } \pm \text { kaudal für RCA-Grafts; für LCA-Grafts ist das Ostium } \\
\text { meist verkürzt in der RAO-Darstellung }\end{array}$ \\
\hline
\end{tabular}

tionierung und die Entfaltung des Stents deutlich [44].

Das „Leopard-Crawl“-Manöver kann bei „unüberwindbaren“ Stenosen mit konventionellen Ballons in Einzelfällen eine Technik darstellen, wie Scoring/Cutting-Ballons (z.B. NSA Alpha) in derartigen Stenosen eingesetzt werden können (s. • Abb. 14).

Obwohl die routinemäßige Anwendung einer Rotablation die Ergebnisse nach DES nicht verbessert, ist sie immer noch für die Behandlung stark verkalkter oder stark fibrotischer Läsionen erforderlich, die vor der Stentimplantation nicht mit einem Ballon passiert oder ausreichend dilatiert werden können (Ilb, C).

Alternativ kann auch die orbitale Atherektomie eingesetzt werden. Ähnlich wie für die Lithoklasten ergeben sich aus bisher nicht randomisierten Studien vielversprechende Ergebnisse. Indikationen und entsprechende Anwendungsgebiete müssen aus randomisierten Daten abgeleitet werden.

\subsection{Erfolgskriterien der $\mathrm{PCl}$}

Ziel der $\mathrm{PCl}$ ist es, eine hämodynamisch wirksame Koronarstenose dauerhaft zu beseitigen, ohne dass es dabei zu einer Myokardschädigung oder zum Verschluss von größeren Seiten- oder Endästen kommt. In der internationalen Literatur sind mehrere koronare Erfolgs- oder Misserfolgskriterien gebräuchlich. Im Rahmen einer Stentimplantation sollte eine Unterexpansion des Stents $>20 \%$ vermieden werden. Bei angiographisch unklarem Befund sollte das Stentergebnis mit der intravaskulären Bildgebung (IVUS, OCT) evaluiert werden (Ila, B).

Für einen prozeduralen Erfolg sollten während des Krankenhausaufenthaltes keine MACE (Tod, Myokardinfarkt, Not- fallbypassoperation) auftreten. Für die 30-Tage-Inzidenz bei koronarer Stentimplantation lassen sich für MACE folgende Prädiktoren identifizieren: Alter, Diabetes mellitus, ACS, Stentlänge, Stentüberlappung, eingeschränkte LV-Funktion sowie als stärkster Prädiktor die residuelle Dissektion. Troponin I- oder T-Erhöhungen nach $\mathrm{PCl}$ durch Mikroembolien, Verschlüsse kleiner Seitenäste, Spasmen u.a. werden nicht als Komplikation gewertet. In den aktuellen Guidelines werden keine seriellen Troponinkontrollen nach $\mathrm{PCl}$ routinemäßig empfohlen.

\subsection{Möglichkeiten der Therapie bei refraktärer Angina pectoris}

Bei therapierefraktärer Angina pectoris ist zunächst eine optimale medikamentöse Therapie entsprechend der europäischen Leitlinie zum chronischen Koronarsyndrom anzustreben [47]. Alternativ kann bei nachgewiesener myokardialer Ischämie im Bereich des Perfusionsgebiets der linken Koronarangiographie (Stress-MRT, Szintigraphie, Stressechokardiographie) eine Koronarsinusreduktion (ReducerTherapie) erwogen werden. Der Reducer ist ein sanduhrförmiger, ballonexpandierbarer Stent aus Edelstahl, der perkutan den Koronarvenensinus implantiert wird. Nach 2 bis 6 Monaten wird hierdurch die Ausbildung einer 60 - bis 70\%igen Stenose im Koronarvenensinus induziert, welche retrograd zu einer Erhöhung des Kapillardruckes führt. Insbesondere im subendokardialen Bereich führt dies zu einer Reduktion des Gefäßwiderstandes mit einer Besserung des in der Ischämie pathologisch erhöhten Verhältnisses zwischen dem subepikardialen und dem subendokardialen Blutfluss. über die rechte $V$. jugularis interna in
Der primäre Endpunkt der randomisierten, sham-kontrollierten COSIRA-Studie war eine Verbesserung der Angina-pectoris-Beschwerden um mindestens 2 CCSKlassen nach 6 Monaten [48]. Dieser Endpunkt wurde bei $35 \%$ der Patienten in der Behandlungsgruppe und bei $15 \%$ der Patienten in der Kontrollgruppe erreicht $(p=0,02)$. Eine Verbesserung um eine CCSKlasse konnte bei $71 \%$ (vs. $42 \%$ in der Kontrollgruppe) der Patienten nach der Implantation des koronaren Reducers beobachtet werden. Weitere Registerstudien zeigen eine Verbesserung der Symptomatik, der linksventrikulären Funktion sowie der linksventrikulären Perfusion bei ca. $75-80 \%$ der behandelten Patienten $[49,50]$.

Geeignete Kandidaten für diese Therapieform stellen Patienten mit einer reversiblen Ischämie im Bereich des linken Ventrikels mit einer linksventrikulären Ejektionsfraktion $>30 \%$ und einem Druck im rechten Vorhof von $<15 \mathrm{~mm} \mathrm{Hg}$ dar, die trotz maximaler Therapie über eine refraktäre Angina pectoris berichten.

\section{Indikation zur PCI bei chronischem Koronarsyndrom}

\section{1 $\mathrm{PCl}$ versus alleinige medikamentöse Therapie}

Verschiedene Studien konnten zeigen, dass eine myokardiale Revaskularisation im Vergleich zu einer rein medikamentösen Therapie Angina-pectoris-Symptome effektiver reduzieren sowie Leistungsfähigkeit und Lebensqualität signifikant steigern kann [51, 52].

Dies gilt insbesondere für Patienten mit 1- oder 2-Gefäß-Erkrankungen, und dieser Vorteil bleibt auch über einen längeren klinischen Verlauf erhalten, wie mehrere Studien und Metaanalysen zeigen konnten [53-57].

Die ACIP-Studie zeigte bei asymptomatischen oder oligosymptomatischen Patienten mit Ischämienachweis sogar eine Reduktion der Letalität durch eine Revaskularisation ( $\mathrm{PCl}$ oder Bypassoperation) während eines 2-Jahres-Zeitraums [52].

In der ORBITA-Studie wurde eine Koronarintervention aufgrund einer 1-GefäßErkrankung mit einer Placeboprozedur verglichen. Hier war 6 Wochen nach me- 


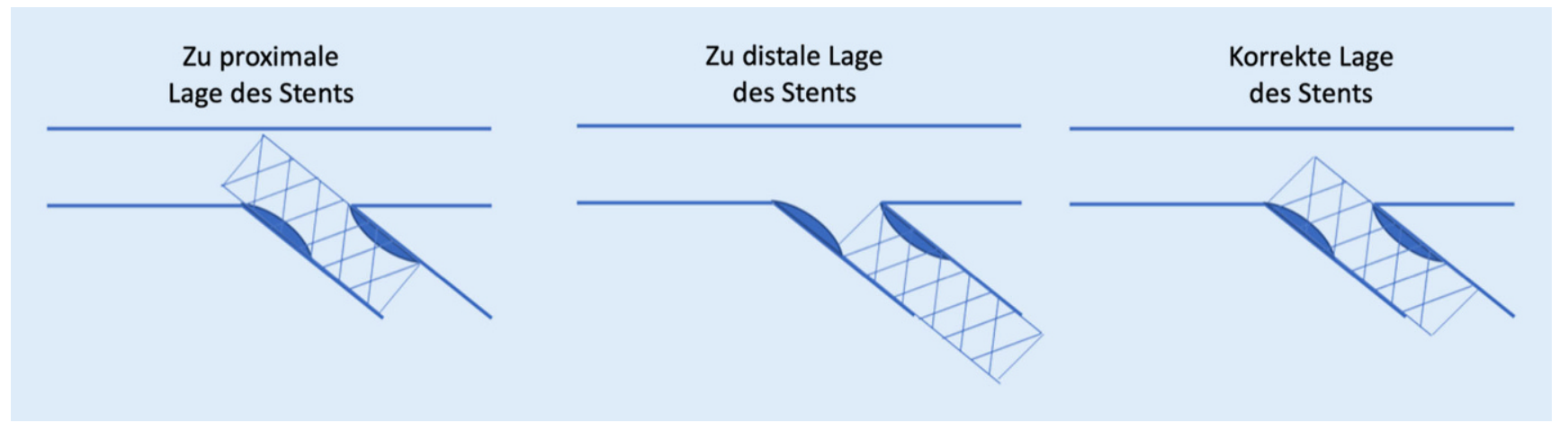

Abb. 9 \ Stentpositionen in ostialen Läsionen

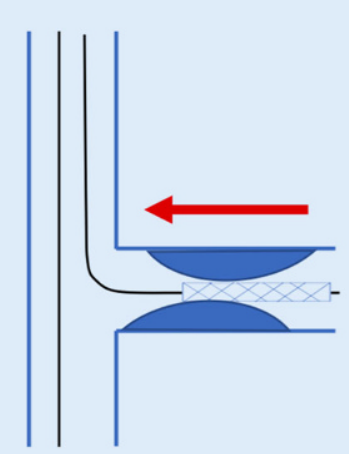

a

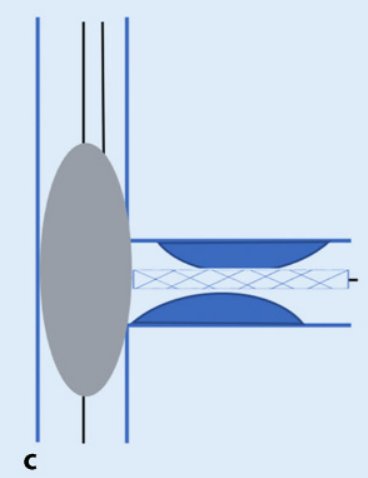

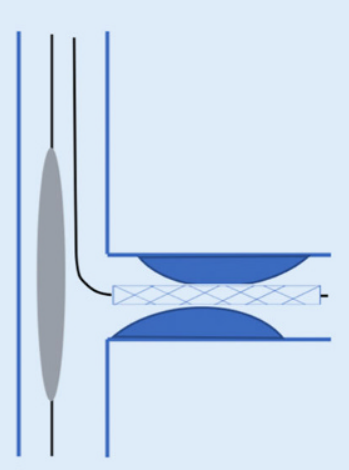

b

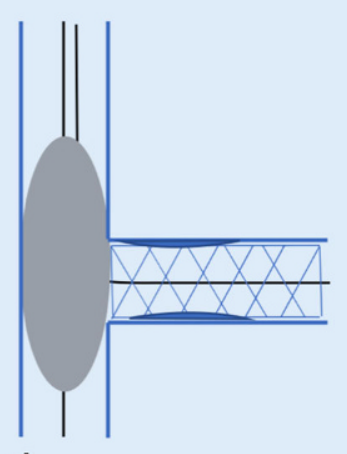

d
Abb. $10<$ Drawback-Technik. a Stent vorgeschoben über die Läsion. b Ballon ist über einen zweiten Draht über dem Ostium des Zielgefäßes platziert und der Stent bis zum Ostium zurückgezogen. cInsufflation des Ballons (6-8 atm) und zurückziehen des Stents gegen den insufflierten Ballon. d Stentimplantation dikamentöser Optimierung und 6 Wochen nach $\mathrm{PCl}$ oder Placeboprozedur kein signifikanter Unterschied im primären Endpunkt, der Zunahme der ergometrischen Belastungszeit, festzustellen. Allerdings zeigte eine im Rahmen der Studie durchgeführte Stressechokardiographie eine Verbesserung des "wall motion score index" nur bei PCI-Patienten [58].

In der FAME 2-Studie konnte außerdem eine deutliche und über 5 Jahre anhaltende Symptomverbesserung nach FFRgeführter $\mathrm{PCl}$ gezeigt werden. Dieser Vorteil für die PCl war statistisch hochsignifikant, obwohl einige Patienten in den medi- kamentösen Behandlungsarm wechselten $[59,60]$.

In der COURAGE-Studie war bei einem hochselektionierten Patientenkollektiv mit stabiler koronarer Herzerkrankung kein Unterschied im klinischen Verlauf und bei den Symptomen festzustellen [61]. Bei allerdings nachgewiesener ausgedehnter Ischämie war auch in COURAGE eine Tendenz zugunsten besserer Ergebnisse in der PCI-Gruppe zu erkennen [62].

Die ISCHEMIA-Studie untersuchte randomisiert an einem hochselektionierten Patientenkollektiv mit stabiler koronarer Herzerkrankung und moderater bis schwe- rer Ischämie in nichtinvasiven Stresstestungen den Unterschied zwischen einer initialen invasiven Strategie und optimaler medikamentöser Therapie über im Mittel 3,2 Jahre an mehr als 5000 Patienten. Dabei konnte hinsichtlich der harten Endpunkte kardiovaskulärer Ereignisse oder Mortalität kein signifikanter Unterschied der beiden Gruppen dokumentiert werden. Hinsichtlich der Symptomatik konnte wie auch in anderen Studien eine signifikante Verbesserung unter Inkaufnahme einer erhöhten Komplikationsrate erreicht werden [63].

Aus den zitierten Studien lässt sich keine generelle Indikation für oder gegen eine interventionelle Behandlungsstrategie oder rein medikamentöse Therapie bei Patienten mit stabiler AP ableiten.

Die Entscheidung für eine $\mathrm{PCl}$ bei $\mathrm{Pa}$ tienten mit chronischem Koronarsyndrom sollte nach individueller Abwägung erfolgen.

Eine $\mathrm{PCl}$ ist bei medikamentös optimal eingestellten Patienten dann sinnvoll, wenn durch die Koronarintervention eine Verbesserung der Symptomatik oder der Prognose zu erwarten ist.

\section{2 $\mathrm{PCl}$ versus $A C B$}

Die Entscheidung bezüglich der Auswahl der Revaskularisationsstrategie bei Patienten mit chronischem Koronarsyndrom sollte unter Berücksichtigung der anatomischen Komplexität der KHK, des chirurgischen Mortalitätsrisikos (STS-Score), der kardialen und extrakardialen Komorbiditäten, der Wahrscheinlichkeit der vollständigen Revaskularisation, aber auch der Patientenpräferenz getroffen werden. 


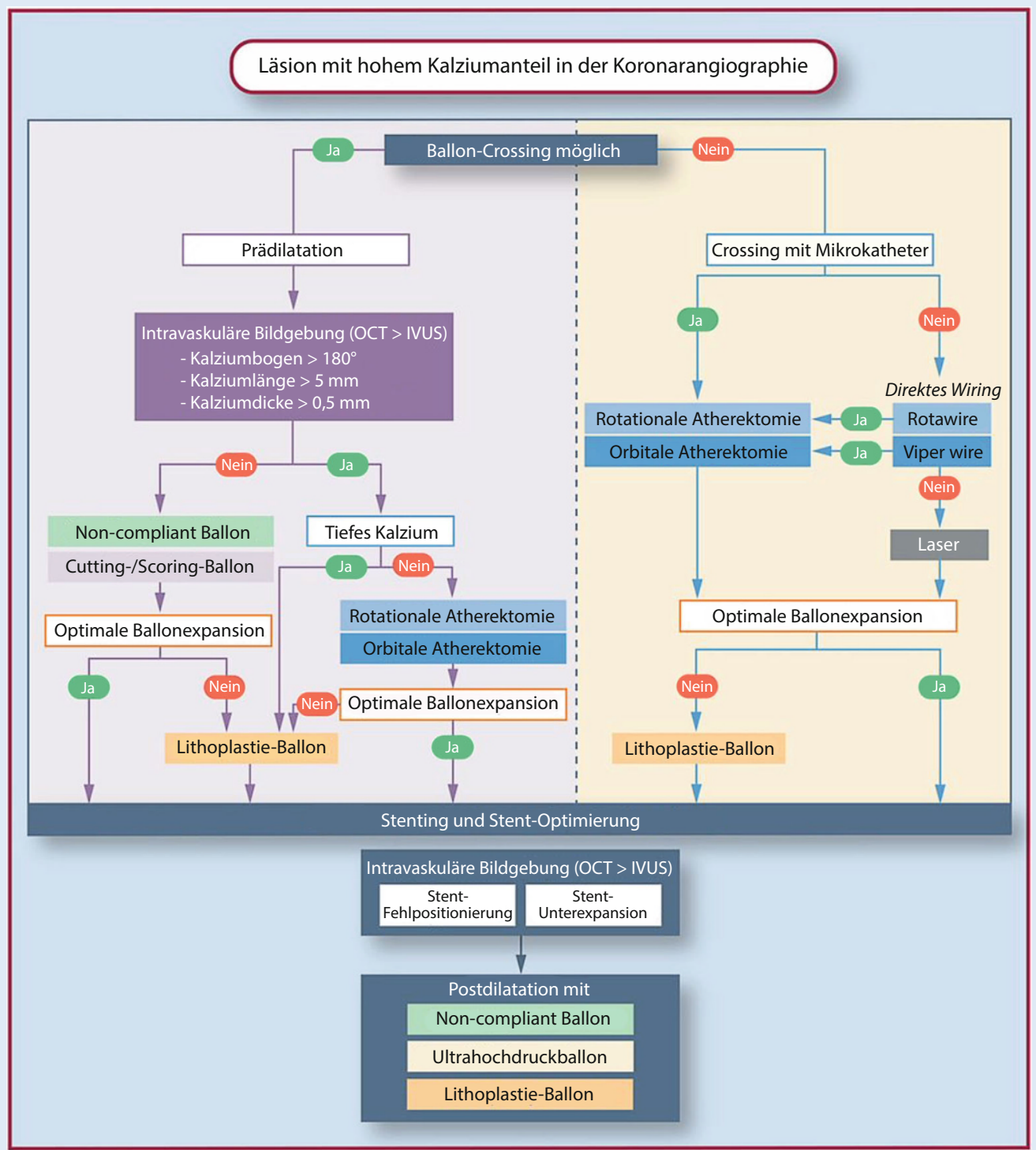

Abb. $11 \triangleleft$ Algorithmuszur Strategieplanung bei kalzifizierten Läsionen. (Mod. nach [43])

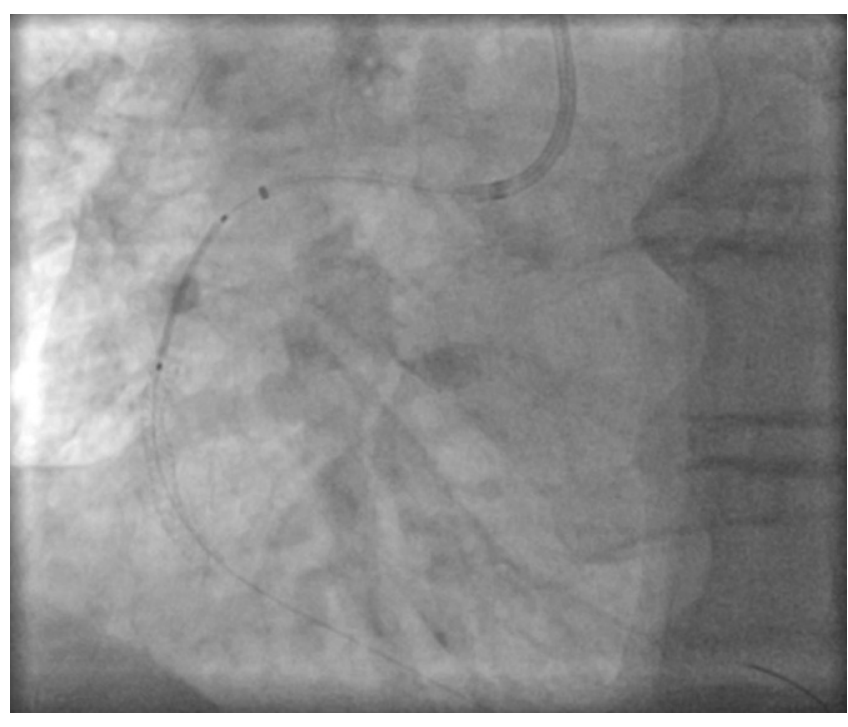

Abb. $12<$ Beispiel für den Einsatz eines sog. Extension-Katheters zur verbesserten Positionierung eines Ballons bzw. Stents

\subsection{Eingefäßerkrankung}

Bei limitierender Symptomatik und/oder Ischämienachweis bzw. Nachweis einer hämodynamisch signifikanten Stenose mittels FFR $(\leq 0,80)$ oder iFR $(\leq 0,89)$ ist die Durchführung einer $\mathrm{PCl}$ bei interventionell Erfolg versprechender Koronaranatomie indiziert $(I, A)[6,64]$.

Bei koronarer 1-Gefäß-Erkrankung und geeigneter Koronaranatomie ist die $\mathrm{PCl}$ einer chirurgischen Revaskularisation vorzuziehen. Es ist zu berücksichtigen, dass LAD/RCX-Abgangsstenosen, Bifurkationsstenosen, interventionell schlecht erreichbare Stenosen und Koronarverschlüsse mit einer $\mathrm{PCl}$ schwieriger zu behandeln sind. 


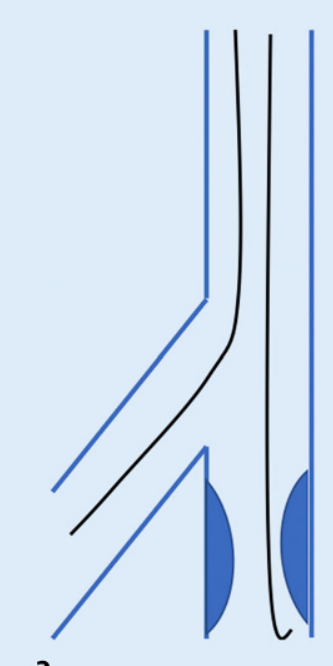

a

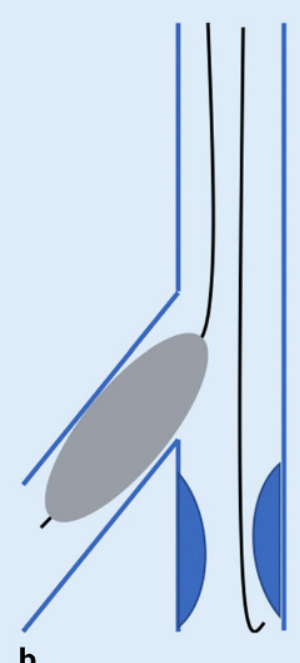

b
Abb. $13 \triangleleft$ AnkerBallon-Technik

\subsection{Mehrgefäßerkrankungen}

Bei Patienten mit KHK sollte das Ziel die komplette Revaskularisation aller hämodynamisch relevanten Stenosen sein. Dies verringert nicht nur die Rate an erneuten Revaskularisationen, sondern senkt im Vergleich zur inkompletten Revaskularisation auch signifikant die Mortalität [65]. Insbesondere ein residualer SYNTAX-Score $>8$ nach einer Intervention sollte aufgrund des schlechteren Outcomes unbedingt vermieden werden [66].

Die BEST-Studie, die ACB und $P C I$ bei Patienten mit Mehrgefäßerkrankung verglich, konnte zeigen, dass die PCl im Vergleich zur ACB-Operation mit erhöhten Raten an inkompletter Revaskularisation sowie an erneuten Revaskularisationen während des Follow-up-Zeitraums verbunden war [67]. In einer großen Metaanalyse stellt sich dieser Vorteil der ACB bei koronarer 3-Gefäß-Erkrankung ohne Hauptstammbeteiligung insbesondere für die Patienten mit hoher anatomischer Komplexität dar [68]. Daher gelten folgende Empfehlungen im Einklang mit den aktuellen Leitlinien der ESC [6]. Bei einer 2-Gefäß-Erkrankung wird die $\mathrm{PCl}$ der $\mathrm{ACB}$ vorgezogen. Bei einer 3-Gefäß-Erkrankung muss zur Entscheidung der optimalen Therapie v. a. die Komplexität der KHK beurteilt werden. Hierzu wird der SYNTAX-Score verwendet (www.syntaxscore.com). Bei einem SYNTAX-Score bis 22 kann die KHK sowohl mit PCl oder mittels ACB-Operati- on behandelt werden. Ab einem SYNTAXScore über 22 ist die ACB-Operation der $\mathrm{PCl}$ vorzuziehen (- Tab. 4).

Die Relevanz der Stenosen sollte bei angiographischen Stenosen zwischen 50 und $70 \%$ und inkonklusivem Ischämienachweis durch die FFR überprüft werden $[6,69,70]$. Teilweise ergibt sich daraus statt einer - angiographisch beurteilten koronaren 3-Gefäß-Erkrankung eine funktionelle koronare 2- oder 1-Gefäß-Erkrankung, ggf. liegen auch gar keine hämodynamisch relevanten Stenosen vor. Dies bewirkt häufig eine komplette Veränderung der Therapieempfehlung. In diesem Zusammenhang ist wichtig, dass die Ergebnisse der FAME II-Studie auch über 5 Jahre eine Verlässlichkeit der FFR-Messungen nachweisen [60].

Selbstverständlich sind bei jeder Entscheidung zwischen verschiedenen Therapieoptionen der Allgemeinzustand und die Komorbiditäten des Patienten zu berücksichtigen und diese auch im HeartTeam zu diskutieren.

\subsection{Stenosen des ungeschützten Hauptstammes der linken Koronar- arterie (Hauptstammstenose)}

Hinsichtlich der Therapie der Hauptstammstenose zeigte eine große Metaanalyse, die 11 randomisierte Studien mit über 11.000 Patienten mit Mehrgefäßerkrankung einschloss, über einen Nachverfolgungszeitraum von 5 Jahren keinen
Unterschied in der Mortalität zwischen Patienten, die mit PCl oder mittels ACBOperation behandelt wurden [68]. In den großen randomisierten Studien zur $\mathrm{PCI}$ vs. ACB bei Hauptstammstenose (SYNTAX, PRECOMBAT, EXCEL) ergaben sich hinsichtlich des kombinierten Endpunktes (Tod, Myokardinfarkt und Schlaganfall) in einem Zeitraum von 5 Jahren nach der Indexprozedur keine Unterschiede zwischen den Verfahren [71-74]. In NOBLE und EXCEL zeigte sich jedoch eine erhöhte Rate an erneuten Revaskularisationen bei den Patienten, die initial mittels der PCI behandelt wurden [75].

Entsprechend ist die $\mathrm{PCl}$ aktuell eine gleichwertige Alternative zur ACB-Operation für die Behandlung der Hauptstammstenose bis zu einem SYNTAX-Score von 0 bis $22(\mathrm{I}, \mathrm{A})$. Ab einem SYNTAX-Score von 23 bis 32 stellt die $\mathrm{PCl}$ aufgrund der erhöhten Anzahl erneuter Revaskularisationen eine Behandlungsalternative dar, die erwogen werden sollte (Klasse Ila, A), ab einem SYNTAX-Score von $\geq 33$ sollte eine ACB-Operation durchgeführt werden $(I, A)$. Eine Übersicht über die Empfehlungen der Leitlinie zur myokardialen Revaskularisierung ist in • Tab. 4 dargestellt.

\subsection{PCl der Restenose/Stenose}

Die In-Stent-Restenose (ISR) liegt trotz konsequenter Anwendung neuester DES bei ca. 5\%. Definitionsgemäß liegt eine ISR dann vor, wenn eine Verengung $>50 \%$ innerhalb des Stents, aber auch jeweils $5 \mathrm{~mm}$ nach distal oder proximal außerhalb des gestenteten Abschnitts liegt (s. Zusatzmaterial Online zusätzliche Abb. 31). Die klinische Definition der ISR umfasst die $50 \%$ Diameterstenose und einen der nachfolgenden Punkte: (1) Angina pectoris, (2) objektive Ischämiezeichen (Wandbewegungsstörungen, EKG) sowie die signifikante Einschränkung der funktionellen Flussreserve (FFR $<0,80$, iFR $<0,89$ ) [76].

Die Behandlung der ISR ist immer noch eine Herausforderung und wird aufgrund der unterschiedlichen Datenlage kontrovers diskutiert. Grundsätzlich erscheint es wichtig, im Vorfeld die Ursache eines Stentversagens (z.B. Unterexpansion, Fraktur oder Malapposition) näher zu evaluieren, weshalb die intravaskuläre Bildgebung erwogen werden kann (Ilb, C) (s. Beispiel in 


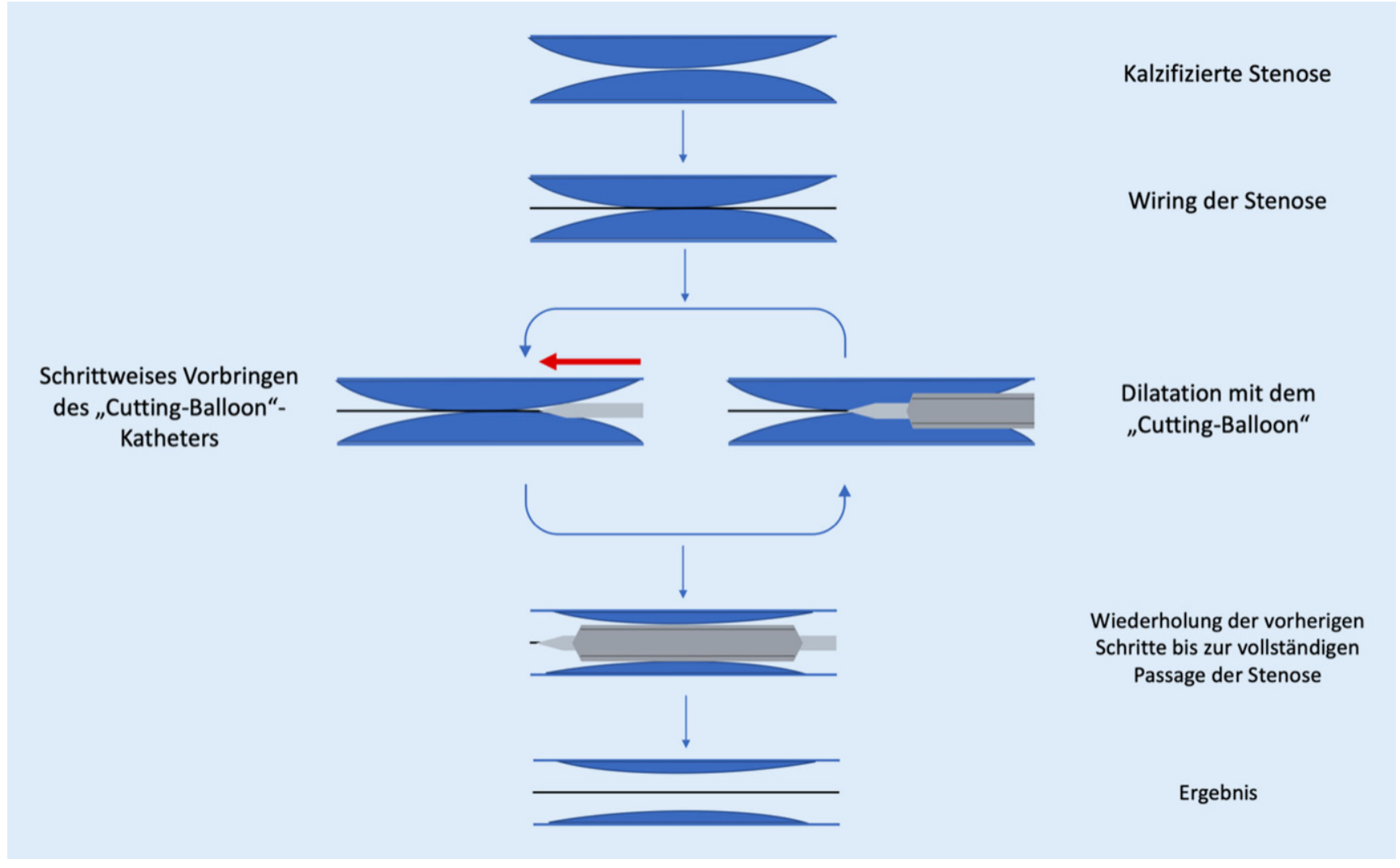

Abb. 14 \ Leopard-Crawl-Technik
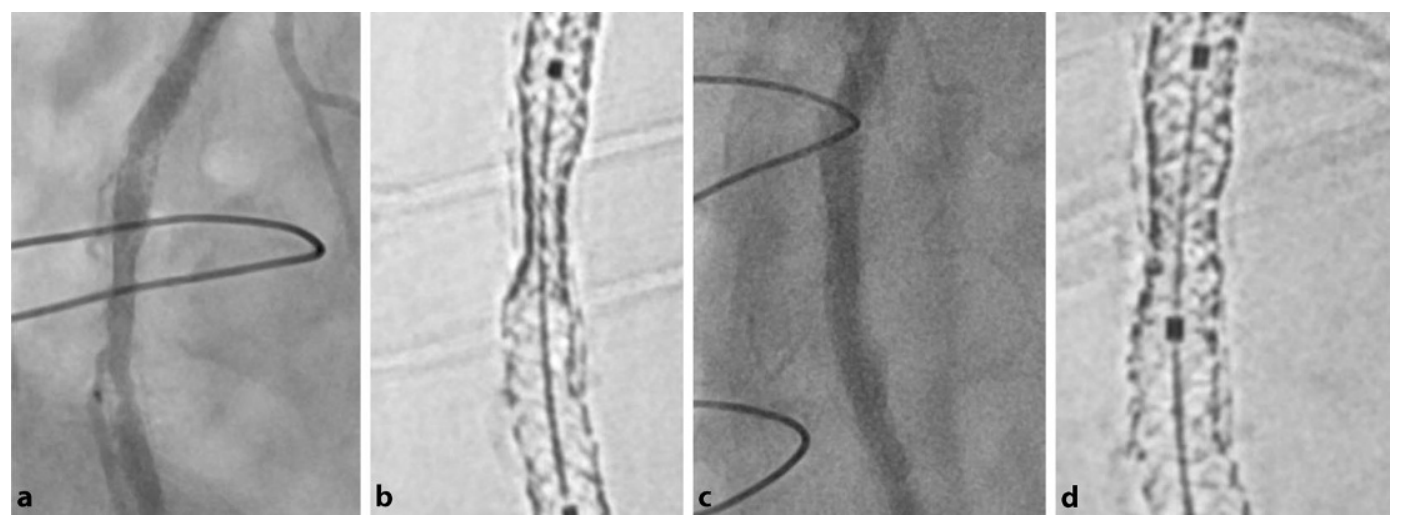

Abb. $15<$ Instentrestenose bei unterexpandiertem Stent $(\mathbf{a}, \mathbf{b})$. Therapie mit Ultrahochdruckballons und Lithoplastie bewirkt angiographisch gutes Ergebnis (c). Im Stent-Enhancement (d) verbleibt eine Unterexpansion

- Abb. 15). Gerade durch die OCT-Untersuchung lässt sich zudem das Vorhandensein einer Neoatherosklerose beurteilen.

\subsection{Diabetiker und koronare 3-Gefäß-Erkrankung}

Mehrere Studien zeigten für eine Bypassoperation bei Diabetikern mit koronarer Mehrgefäßerkrankung eine geringere Gesamtmortalität im Vergleich zu einer PCI $[77,78]$.

Die FREEDOM-Studie konnte einen klaren Vorteil für die Bypassoperation im Ver- gleich zur $\mathrm{PCl}$ hinsichtlich des primären Endpunktes (jedweder Tod, nichtfataler MI und Schlaganfall) nach 5 Jahren dokumentieren [79].

In der BEST-Studie (77\% der untersuchten Patienten hatten eine koronare 3-Gefäß-Erkrankung), in der die Bypassoperation mit der $\mathrm{PCl}$ mit Everolimus-ElutingStents verglichen wurde, zeigte sich bei $\mathrm{Pa}$ tienten mit Diabetes eine höhere Mortalität in der PCl- im Vergleich zur Bypassgruppe $(15,5 \%$ vs. $10 \% ; p=0,0004)$. Das Mortalitätsrisiko nach einer $\mathrm{PCl}$ stieg weiter in $\mathrm{Ab}$ hängigkeit der Komplexität der Koronar- anatomie, die mithilfe des SYNTAX-Scores evaluiert wurde (SYNTAX 0-22: 10,5\% vs. $8,4 \%, p=0,57$; SYNTAX 23-32: $14,0 \%$ vs. $9,5 \%, p=0,0129 ;$ SYNTAX $>32: 19,2 \%$ vs. $11,2 \%, p=0,0094$ ) [67].

Aufgrund dieser Daten geben die aktuellen ESC-Leitlinien eine I, A-Empfehlung für eine Bypassoperation bei Patienten mit Diabetes mellitus und intermediärem oder hohem SYNTAX-Score $>22$ und eine III, AEmpfehlung für die $\mathrm{PCl}[6]$. 
Tab. 4 Empfehlungen zur Revaskularisation [6]

\begin{tabular}{|c|c|c|c|c|}
\hline Empfehlung & \multicolumn{2}{|c|}{ CABG } & \multicolumn{2}{|c|}{$\mathrm{PCl}$} \\
\hline \multicolumn{5}{|l|}{ 1-Gefäß-KHK } \\
\hline Ohne proximale LAD-Stenose & $\mathrm{Illb}$ & $\mathrm{C}$ & 1 & $\mathrm{C}$ \\
\hline Mit proximaler LAD-Stenose & I & A & 1 & A \\
\hline \multicolumn{5}{|l|}{ 2-Gefäß-KHK } \\
\hline Ohne proximale LAD-Stenose & $\mathrm{Ilb}$ & C & I & $\mathrm{C}$ \\
\hline Mit proximaler LAD-Stenose & I & B & I & $\mathrm{C}$ \\
\hline \multicolumn{5}{|l|}{ Hauptstamm-KHK } \\
\hline Hauptstamm-KHK mit niedrigem SYNTAX-Score (0-22) & I & A & I & A \\
\hline $\begin{array}{l}\text { Hauptstamm-KHK mit intermediärem SYNTAX-Score } \\
(23-32)\end{array}$ & I & A & Illa & A \\
\hline Hauptstamm-KHK mit hohem SYNTAX-Score $(\geq 33)$ & I & A & III & B \\
\hline \multicolumn{5}{|l|}{ 3-Gefäß-KHK ohne Diabetes } \\
\hline 3-Gefäß-KHK mit niedrigem SYNTAX-Score (0-22) & 1 & A & I & A \\
\hline 3-Gefäß-KHK mit intermediärem SYNTAX-Score (23-32) & I & A & III & A \\
\hline \multicolumn{5}{|l|}{ 3-Gefäß-KHK mit Diabetes } \\
\hline 3-Gefäß-KHK mit niedrigem SYNTAX-Score (0-22) & 1 & A & $\mathrm{Ilb}$ & A \\
\hline 3-Gefäß-KHK mit intermediärem SYNTAX-Score (23-32) & 1 & A & III & A \\
\hline
\end{tabular}

\subsection{PCI bei hohem CABG-Risiko}

Patienten mit hohem operativem Mortalitätsrisiko profitieren ggf. eher von einem interventionellen oder konservativen Vorgehen. Um das operative Risiko zu erfassen, existieren etablierte Risiko-ScoringSysteme wie der STS- und Euroscore [80, 81].

Der STS-Score erfährt auf der Basis laufender (Register-)Studien regelmäßige Updates, der Euroscore II ist jedoch als gleichwertig für die Evaluation des operativen Mortalitätsrisikos bei Bypassoperationpatienten zu betrachten.

Der ältere logistische Euroscore ist ebenfalls ein mögliches Instrument, die perioperative Mortalität abzuschätzen, überschätzt das Mortalitätsrisiko im Vergleich zum Euroscore II aber ungefähr um mindestens das Doppelte [82].

Obwohl diese Scores für die Risikoeinschätzung hilfreich sind, lässt keiner der Scores eine perfekte Risikoabschätzung zu: Sowohl STS- als auch Euroscore sind aufgrund der zugrunde liegenden Methodik, der benutzten spezifischen Definitionen, fehlender klinisch wichtiger Variablen (z. B. Gebrechlichkeit/Fraility), der umständlichen Score-Berechnung und der beschränkten externen Validierung limitiert.

Die Kenntnis operativer Risiko-Scores ist für die Einzelfallbetrachtung und -dis- kussion jedoch unabdingbar. Diese ersetzen jedoch nicht die auf regelmäßiger Basis stattfindende interdisziplinäre kardiologisch-kardiochirurgische Konferenz (Heart-Team) in der individualisierte Behandlungsoptionen für den einzelnen Patienten geprüft werden.

\subsection{PCl von venösen Bypassgefäßen}

Stenosen oder Verschlüsse venöser Bypassgefäße können einerseits nahtassoziiert (frühzeitig nach Operation) oder andererseits degenerativ (spät nach Operation) bedingt sein. Die Indikation sowie die Stentwahl entsprechen im Wesentlichen den Empfehlungen zur PCl bei Ein- und Mehrgefäßerkrankung.

Die Restenoserate nach $\mathrm{PCl}$ ist in Bypassgefäßen jedoch wesentlich höher als bei nativen Koronargefäßen [83]. Entsprechend sollte - wenn irgendwie möglich statt einer Intervention am Bypassgefäß die $\mathrm{PCl}$ des nativen Koronargefäßes bevorzugt werden (Ila, C). Sollte dies nicht möglich sein, erfolgt die Intervention am Bypassgefäß. Die Intervention an einem venösen Bypassgraft ist aufgrund des höheren Embolierisikos stets eine Hochrisikoprozedur. Im Einzelfall kann die Evaluation der Bypassstenose mittels intravaskulärer Bildgebung erwogen werden (Ilb, C). Eine Rekanalisation eines chronisch verschlossenen SVG-Grafts sollte aufgrund der schlechten klinischen Ergebnisse nicht durchgeführt werden (III, C) [84].

\subsection{Chronische Verschlüsse}

Ein chronischer Koronarverschluss (CTO) definiert sich als ein kompletter Verschluss der Koronararterie von einer Dauer von mehr als 3 Monaten [31]. CTOs liegen bei ca. 15-25\% der Patienten mit einer angiographisch gesicherten KHK vor [31, 85, 86].

Die interventionelle Wiedereröffnung einer CTO wird immer noch kontrovers diskutiert. Eine systematische Analyse von 25 Beobachtungsstudien assoziierte eine erfolgreiche Rekanalisation einer CTO mit verbessertem klinischem Outcome [87]. Allerdings konnte keine der beiden großen randomisierten Studien (EURO-CTO [ $n=396]$ und DECISION-CTO $[n=834])$ einen Vorteil für die Rekanalisation der CTO hinsichtlich MACE, Tod oder Myokardinfarkt im Vergleich zur medikamentösen Therapie zeigen. In der EURO-CTO-Studie zeigten Patienten, die rekanalisiert wurden, jedoch klinische Vorteile wie eine Verbesserung der körperlichen Leistungsfähigkeit sowie eine Verminderung der Symptomatik [88, 89].

In den letzten Jahren ist durch die Weiterentwicklung von Material und Techniken sowie eine zunehmenden Expertise die Erfolgsrate der Rekanalisationen stark angestiegen und beträgt aktuell - abhängig von der Erfahrung der Operateure ca. $80-90 \%[31,85,88]$. Dennoch muss aufgrund der kontroversen Datenlage und des zeitlichen und apparativen Aufwands der Prozedur die Indikation zur interventionellen Revaskularisation einer CTO streng gestellt werden. Eine Indikation besteht bei Nachweis von Vitalität des Myokards in Kombination mit entsprechender Symptomatik trotz optimaler medikamentöser antianginöser Therapie bzw. bei asymptomatischen Patienten, die eine belastungsinduzierte Ischämie von $>10 \%$ des linksventrikulären Myokards aufweisen (Ila, B) [31]. Hinsichtlich der Aussicht auf Erfolg eines interventionellen Vorgehens helfen unterschiedliche Scoring-Modelle, wie z. B. der J-CTO-Score [90]. Auch ein präinterventionelles $C T$ kann hilfreich sein, um die Länge der Verschlussstrecke, den Gefäß- 

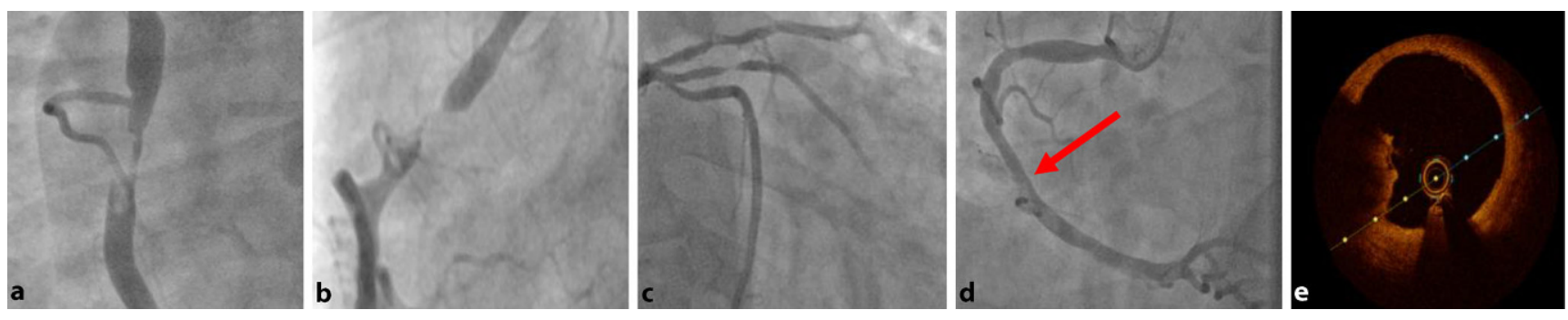

Abb. 16 \ Beispiele für sog. „culprit lesions" bei Patienten mit NSTE-ACS: a sichtbarer Thrombus, b subtotale Stenose mit unregelmäßigem Plaque, c Stenose mit poststenotisch vermindertem Fluss, d unklarer angiographischer Befund (roter Pfeil), e Nachweis eines Thrombus im OCT

verlauf sowie den Grad der Verkalkung zu antizipieren (Ila, C).

\subsection{PCl bei speziellen Situationen}

\subsubsection{PCl vor größeren}

\section{nichtkardialen Operationen}

Das Vorgehen erfordert eine Einzelfallbetrachtung, da eine generelle Indikation zur $\mathrm{PCl}$ oder Bypassoperation z.B. vor einer elektiven nichtkardialen vaskulären Operation nicht besteht. Falls eine $\mathrm{PCl}$ vor der geplanten Operation durchgeführt wird, ist das verwendete interventionelle Verfahren nach Möglichkeit anzupassen z.B. durch die Verwendung von Stents, die nur eine kurze Dauer der Thrombozytenaggregationshemmung notwendig machen. Die duale Thrombozytenaggregationshemmung sollte auf keinen Fall unkritisch früh nach einer erfolgten $\mathrm{PCl}$ abgesetzt werden [91].

\subsubsection{PCl im Alter}

Bei alten Patienten gelten grundsätzlich die gleichen Indikationen zur Revaskularisation wie bei jungen Patienten mit KHK. Problematisch ist jedoch das häufig erhöhte Blutungsrisiko (PRECISE-DAPT > 25). Ziel sollte daher sein, das Blutungsrisiko gering zu halten und daher die Dauer der dualen Plättchenhemmung möglichst kurz zu halten. Dennoch sollten auch bei den älteren Patienten primär DES-Stents implantiert werden, da diese ein verbessertes Outcome im Vergleich zu BMS auch bei einer verkürzten Dauer der DAPT von 1 Monat haben [92-94].

\section{Indikation zur Katheter- intervention $(\mathrm{PCl})$ beim akuten Koronarsyndrom (ACS)}

\subsection{Zugangsweg beim ACS}

Der radiale Zugangsweg sollte bei Patienten mit ACS bevorzugt werden $(I, A)[6,45$, 46]. Die Studien RIFLE-STEACS und LADL zeigten einen Vorteil des radialen gegenüber dem femoralen Zugang zumindest für Subgruppen $[95,96]$. Die sehr prominente MATRIX-Studie - $47 \%$ der eingeschlossenen Patienten hatten einen ST-Hebungsinfarkt - wies in der 30-Tages-Analyse einen Überlebensvorteil für Patienten nach, die im ACS radial behandelt wurden [97]. In Metaanalysen wurde ebenfalls ein Überlebensvorteil durch den radialen Zugang beim ACS gezeigt [98], und in den Leitlinien ist dies entsprechend verankert. Es sei allerdings einschränkend erwähnt, dass nach 1 Jahr dieser Vorteil des radialen Zugangs zumindest in der MATRIX-Studie nicht mehr nachvollziehbar war. Trotzdem überwog der klinische Benefit für den radialen im Vergleich zum femoralen Zugang [99].

Vermutlich sind die positiven Ergebnisse der großen Studien zum radialen Zugang im ACS auch durch die zum Zeitpunkt der Studie hohe Verwendungsrate an GPIllb/llla-Rezeptorantagonisten mit bedingt, was nicht mehr dem heutigen Standard entspricht. Entsprechend konnte die kürzlich publizierte SAFARI-STEMIStudie keinen Vorteil des radialen Zugangs bei 2212 Patienten mit ST-Hebungsinfarkt hinsichtlich Tod nachweisen. Numerisch zeigten sich zwar weniger Blutungen bei Patienten mit radialem Zugang, allerdings konnte aufgrund des vorzeitigen Abbruchs der Studie kein signifikanter Vorteil dokumentiert werden [100].

Zudem ergeben sich Aspekte, wie z.B. das Vorliegen von Bypass-Grafts, die Notwendigkeit der Anlage eines passageren Schrittmachers oder eines extrakorporalen kardialen Unterstützungssystems bei Patienten im kardiogenen Schock, die manchmal für die primäre Wahl eines transfemoralen Zugangs sprechen. Ein sicheres Beherrschen beider Zugangswege ist daher unabdingbar. Der transradiale Zugang sollte gerade in Situationen mit hohem Blutungsrisiko bevorzugt werden, wenn die entsprechende Expertise vorliegt.

\subsection{Identifikation der "culprit lesion“}

Bei Patienten mit ACS und Mehrgefäßerkrankung sollte zunächst versucht werden, die "culprit lesion“ zu identifizieren, um somit die Läsion zu erkennen, welche der sofortigen Behandlung bedarf. Bei einem Patienten mit STEMI gestaltet sich dies meist einfach, bei einem Patienten, der sich mit einem NSTE-ACS präsentiert, kann dies schwieriger sein. Nichtinvasive sowie invasive Kriterien können Aufschluss geben.

\subsubsection{Elektro- und echokardio- graphische Kriterien}

Veränderungen im EKG können auch bei Patienten mit NSTE-ACS auf die "culprit lesion" hinweisen. ST-Senkungen in V4-V6 sprechen hierbei für eine Läsion in der LAD, während ST-Senkungen in V2-V3 häufig mit einer Läsion im Bereich des RCX einhergehen [101]. Diffuse ST-Senkungen mit einer isolierten ST-Hebung $\geq 1 \mathrm{~mm}$ in der Ableitung aVR sprechen für eine Beteiligung des Hauptstammes oder der proximalen LAD [102, 103]. Das EKGkann jedoch 
Hier steht eine Anzeige.

黑 Springer 


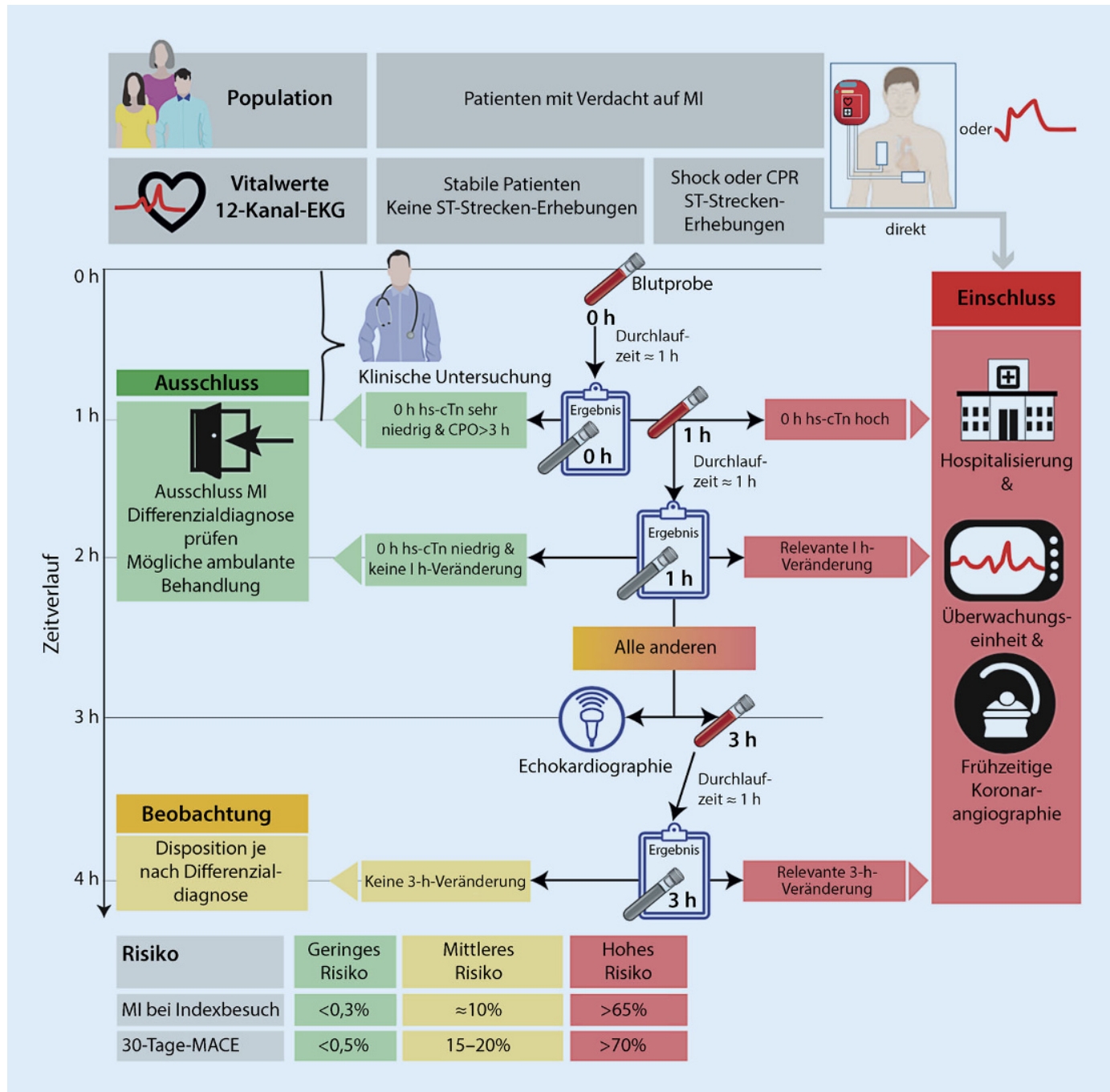

Abb. $17 \triangleleft$ Rule-in- und Rule-out-Algorithmus beim NSTE-ACS. (Mod. nach [45])

sowohl bei einer Mehrgefäß-KHK wie auch bei distalen Läsionen inkonklusiv sein. Gegebenenfalls liegen keinerlei EKG-Veränderungen vor. Die Echokardiographie kann durch die Identifikation von regionalen Wandbewegungsstörungen Hinweise auf die "culprit lesion“ liefern [104].

\subsubsection{Angiographische Kriterien}

Eine "culprit lesion“ ist durch einen intraluminalen Füllungsdefekt und durch mindestens 2 der folgenden Kriterien definiert (s. auch - Abb. 16; [104]):

- Thrombus,

- Plaque Irregularität,

- verminderter Fluss,

- Plaqueulzeration,

- Dissektion.

Bei $40 \%$ der Patienten mit NSTE-ACS findet sich die "culprit lesion" im Bereich der LAD [105]. Bei ca. $25 \%$ der Patienten mit
NSTE-ACS liegt ein kollateralisierter Verschluss einer Koronararterie vor [106, 107]. Bei $40 \%$ erfüllt mehr als eine Läsion diese Kriterien der "culprit lesion" [108-111]. Bei unklaren Befunden kann eine koronare Bildgebung mittels OCT oder IVUS hilfreich sein [104]. Während die FFR für die Identifikation weiterer behandlungsbedürftiger Stenosen zusätzlich zur "culprit lesion" von Nutzen sein kann [112], ist die Rolle der FFR zur Identifikation der "culprit lesion“ bisher nur mangelhaft untersucht [104]. Die durch den Infarkt bedingte mikrovaskuläre Dysfunktion könnte, so wird vermutet, die Ergebnisse verfälschen und eine Interpretation somit erschweren.

\subsection{PCI beim NSTE-ACS}

\subsubsection{Indikation}

Auch im Rahmen eines NSTE-ACS sollte eine eindeutige Evidenz für eine myokardiale Ischämie vor der Koronarangiographie vorliegen. Vergleiche von Routinekoronarangiographien bei allen Patienten mit NSTE-ACS zu selektionierten Patienten mit NSTE-ACS und hoher objektiver Vortestwahrscheinlichkeit für eine pathologische Stenose wurden in vielen RCTs und Metaanalysen angestellt. Dabei konnte gezeigt werden, dass nur für Hochrisikokollektive Endpunkte positiv beeinflusst wurden.

Deshalb ist eine Risikostratifizierung (Troponinbestimmung) obligat. Genaue Verfahrensanweisungen sowie Diagnostikalgorithmen bietet die ESC-Leitlinie zum NSTE-ACS von 2020 (s. - Abb. 17; [45]). 
Tab. 5 Indikationen zur Koronarangiographie beim akuten Koronarsyndrom

Eine sofortige invasive Strategie $(<2 h)$ wird empfohlen bei Patienten mit mindestens einem der folgenden Hochrisikokriterien

\begin{tabular}{|c|c|}
\hline Hämodynamische Instabilität oder kardiogener Schock & \multirow[t]{6}{*}{$\mathrm{I}, \mathrm{C}$} \\
\hline $\begin{array}{l}\text { Wiederkehrende oder anhaltende Angina pectoris, die auf eine medikamentöse Be- } \\
\text { handlung nicht anspricht }\end{array}$ & \\
\hline Lebensbedrohliche Arrhythmien & \\
\hline Mechanische Komplikationen des MI & \\
\hline Akute Herzinsuffizienz, die direkt mit dem NSTE-ACS in Verbindung steht & \\
\hline $\begin{array}{l}\text { ST-Strecken-Senkungen > } 1 \text { mm/6 Ableitungen plus ST-Strecken-Hebungen in aVR } \\
\text { und/oder V1 }\end{array}$ & \\
\hline \multicolumn{2}{|l|}{$\begin{array}{l}\text { Eine frühzeitige invasive Strategie }(<24 \mathrm{~h}) \text { wird empfohlen bei Patienten mit mindestens ei } \\
\text { nem der folgenden Risikokriterien }\end{array}$} \\
\hline Bestätigtes NSTEMI (z. B. mit dynamischem Troponin) & \multirow[t]{4}{*}{$\mathrm{I}, \mathrm{A}$} \\
\hline $\begin{array}{l}\text { Dynamische ST-Strecken oder T-Wellen-Änderungen (symptomatisch oder asympto- } \\
\text { matisch) }\end{array}$ & \\
\hline ROSC ohne ST-Strecken-Hebung oder kardiogenen Schock & \\
\hline GRACE-Score $>140$ & \\
\hline $\begin{array}{l}\text { Eine invasive Strategie beim Ausbleiben von wiederkehrenden Beschwerden oder } \\
\text { dem Fehlen jeglicher der oben genannten Hoch-/Risikokriterien sollte im Rahmen } \\
\text { der CCS-Leitlinien von } 2019 \text { erfolgen [47] }\end{array}$ & $I, A$ \\
\hline
\end{tabular}

Sofortige invasive Strategie $(<\mathbf{2} \mathbf{h})$. Analog zu den STEMI-Algorithmen sollten Hochrisikopatienten mit hämodynamischer Instabilität, wiederkehrender oder anhaltender auf Medikamente resistenter Angina pectoris, lebensbedrohlichen Arrhythmien, mechanischen Komplikationen, akuter Herzinsuffizienz oder spezifischen EKG-Veränderungen einer sofortigen invasiven Strategie $(<2 \mathrm{~h})$ zugeführt werden. Aus Zentren, in denen eine 24/7h-Katheterbereitschaft nicht gegeben ist, sollte eine sofortige Verlegung erfolgen (s. - Tab. 5) (l, C) [45].

Frühe invasive Strategie $(<\mathbf{2 4} \mathbf{h})$. Basierend auf der aktuellen Evidenz, scheint eine frühe invasive Strategie $(<24 \mathrm{~h})$ beim NSTE-ACS nur bei erhöhtem individuellem Risiko einen positiven Effekt zu haben. Hierzu gehören Patienten mit bestätigtem NSTEMI (z. B. aufgrund einer dynamischen hs-Troponin-Kinetik nach $1 \mathrm{~h}$ bzw. $3 \mathrm{~h}$ ), mit dynamischen ST-Strecken oder T-WellenVeränderungen, einem ROSC nach Reanimation ohne ST-Strecken-Hebungen oder kardiogenem Schock und einem erhöhten allgemeinen Risikoprofil, gemessen anhand des GRACE-Scores mit > 140 Punkten (s. - Tab. 5) (l, A) [45].
Selektive invasive Strategie. Bei allen anderen Patienten, die keines der oben genannten Hochrisikokriterien erfüllen, sollte eine invasive Strategie nur nach nichtinvasiver Diagnostik mit vorausgegangener Ischämietestung (Stressechokardiographie oder Stress-MRT) erfolgen (s. - Tab. 5). Durch die Einführung des hs-Troponin sowie die Einführung von Diagnosealgorithmen können auch kleinere Myokardinfarkte präzise detektiert werden. Im Falle eines Ausschlusses durch diese Werkzeuge sollte somit eine an der ehesten chronischen Situation oder eine andere Ursache der Angina pectoris angenommen werden und anhand der Algorithmen der ESC-Leitlinien von 2019 zum CCS vorgegangen werden (s. Kap. 2) (I, A) $[45,47]$.

\subsubsection{Revaskularisationsstrategie bei NSTE-ACS}

Während die sofortige Behandlung der "Culprit lesion" bei der interventionellen Versorgung von Patienten mit ACS im Vordergrund steht, ist die Festlegung einer Strategie bei Patienten mit MehrgefäßKHK unter Umständen herausfordernd.

Revaskularisation bei Mehrgefäß-KHK. Nach Behandlung der "culprit lesion“ zeigt eine komplette Revaskularisation Vorteile gegenüber einem konservativen Procedere [113-115]. Verbleibende behandlungsbedürftige Stenosen haben einen negativen Einfluss auf das Überleben $[116,117]$. Der Zeitpunkt der Vervollständigung der Revaskularisation kann und sollte beim Patienten mit NSTE-ACS vom Untersucher allerdings individuell festgelegt werden. Dies kann eine sofortige komplette Revaskularisation, eine Revaskularisation in mehreren separaten Eingriffen oder eine Revaskularisation mittels Bypassoperation (CABG) bedeuten (I, B) [45].

Hinsichtlich eines direkten Vergleiches zwischen $\mathrm{PCl}$ und $\mathrm{CABG}$ bei Patienten mit NSTE-ACS gibt es keine randomisierten Studien. Die hierzu vorliegenden Daten stammen aus sog. „Propensity-Score-Analysen“, z. B. dem ACUITY Trial. Hier zeigte sich kein Unterschied hinsichtlich Mortalität zwischen Patienten, die mit einer CABG oder einer $\mathrm{PCl}$ behandelt wurden. Mit PCl behandelte Patienten erlitten seltener einen Schlaganfall, einen Myokardinfarkt, Blutungen oder ein akutes Nierenversagen, wiesen jedoch eine erhöhte Anzahl an ungeplanten Revaskularisationen innerhalb eines Jahres auf [118]. Insgesamt werden ca. 5-10\% der Patienten mit einem NSTE-ACS mit CABG behandelt - mit sinkender Tendenz über die letzten Jahre [119]. Ausschlaggebend für die Entscheidung zwischen PCI und CABG sind einerseits die Komplexität der Koronarstenosen (SYNTAX-Score, s. Zusatzmaterial Online zusätzliche Abb. 29), aber auch Komorbiditäten (z. B. Diabetes mellitus, kardiale Voroperationen, Niereninsuffizienz, s. Kap. 5 des Beitrags Manual der Arbeitsgruppe Interventionelle Kardiologie [AGIK] der Deutschen Gesellschaft für Kardiologie-Herz-und Kreislaufforschung e.V. [DGK]. Teil 1: „Durchführung der diagnostischen Herzkatheteruntersuchung" [2]) sowie der Allgemeinzustand des Patienten. Falls sich für eine operative Versorgung mittels CABG entschieden wird, sollte der bestmögliche Zeitpunkt der Operation individuell evaluiert werden. Außerdem sollten für stabile Patienten im Rahmen der Entscheidungsfindung auch die Kriterien aus der ESC/EACTS-Leitlinie von 2018 zur myokardialen Revaskularisation herangezogen werden [6]. Laut europäischer Leitlinien sollten Patienten mit einer entsprechenden Koronarmorphologie und 


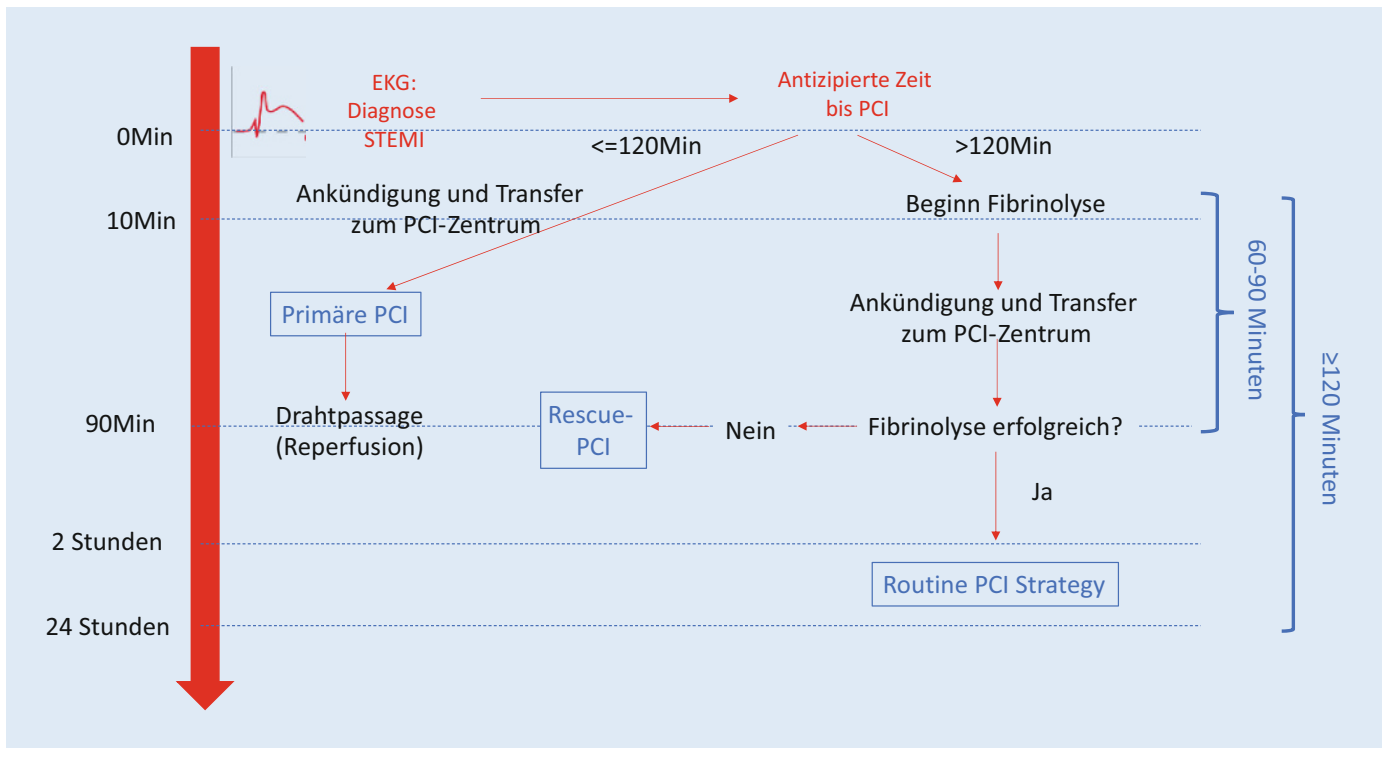

Abb. $18 \triangleleft$ „Strategy Clock": Entscheidungsbaum zur Reperfusionsstrategie in Abhängigkeit der Transferzeiten und des Erfolges der jeweiligen Therapie. (Adaptiert nach [46]) hämodynamischer Instabilität oder anhaltender myokardialer Ischämie ohne Zeitverzug operiert werden $(\mathrm{l}, \mathrm{C})$ [45].

\subsection{PCI bei ST-Hebungsinfarkt (STEMI)}

\subsubsection{Indikation}

Die Indikation zur Katheterintervention wird bei Patienten mit einem STEMI anhand des Beschwerdebeginns und der daraus resultierenden Zeitspanne bis zum "first medical contact" (FMC) sowie anhand der implizierten Zeitspanne des FMC zur PCl festgelegt.

Beschwerdebeginn $\leq 12 \mathrm{~h}$ vor "first medical contact". Die primäre $\mathrm{PCl}$ ist die bevorzugte Reperfusionsstrategie bei Patienten mit STEMI, solange der Symptombeginn zum Zeitpunkt des FMC nicht mehr als $12 \mathrm{~h}$ zurückliegt $(\mathrm{I}, \mathrm{A})$. Randomisierte Studien haben für diesen Zeitraum einen klaren Vorteil der $\mathrm{PCl}$ gegenüber der Fibrinolyse hinsichtlich Mortalität, Reinfarkt und Schlaganfall gezeigt [120-123]. Erst kürzlich konnte im FITT STEMI Trial erneut bewiesen werden, dass jegliche Verkürzung der Zeit zur PCl für den Patienten von Vorteil ist [124]. Entsprechend überwiegen hier auch klar die Vorteile der PCI gegenüber der operativen Revaskularisierung. Es sollte ein direkter Transport der Patienten in das Herzkatheterlabor erfolgen, um jeglichen Zeitverlust zu vermeiden.
Hinsichtlich der maximal tolerierten Zeitverzögerung zwischen der Diagnose STEMI und PCI legen die ESC-Leitlinien von 2017 eine Zeit von 120 min fest (s. - Abb. 18; [46]). Kann die PCl in dieser Zeit nicht durchgeführt werden, sollte so früh wie möglich und spätestens $10 \mathrm{~min}$ nach der STEMI-Diagnose die Fibrinolyse begonnen werden ( $I, A)$. Unabhängig von der initialen Therapiestrategie wird für die optimale weitere Versorgung die direkte Aufnahme oder Verlegung des Patienten in ein $\mathrm{PCl}$-Zentrum empfohlen (I, A) (s. D Abb. 18).

Wenn die ST-Hebungen sich 60-90 min nach der Fibrinolyse nur weniger als 50\% zurückbilden, der Patient hämodynamisch oder elektrisch instabil wird oder weiter über Beschwerden klagt, gilt die Fibrinolyse als nicht erfolgreich, und es sollte eine sofortige Rescue-PCI durchgeführt werden (I, A; [46]).

Auch im Falle einer erfolgreichen Fibrinolyse sollte innerhalb von 2-24h eine Koronardiagnostik erfolgen („Routine PCl Strategy"). Dies verringert die Rate an ReInfarkten und an erneuten myokardialen Ischämien [125, 126]. Die "Strategy Clock“ ist in - Abb. 18 zusammengefasst.

Beschwerdebeginn mehr als $12 \mathrm{~h}$ vor "first medical contact". Bei Patienten mit Beschwerdebeginn von über $12 \mathrm{~h}$ vor $\mathrm{FMC}$ sollte eine primäre $\mathrm{PCl}$ angestrebt werden, wenn zumindest einer der folgenden Punkte zutrifft $[6,46]$ :
- bleibende Zeichen einer myokardialen Ischämie im EKG $(I, C)$,

- persistierende Angina pectoris und dynamische EKG-Veränderungen $(I, C)$,

- persistierende Angina pectoris und hämodynamische oder elektrische Instabilität (I, C),

- bislang asymptomatischer Patient mit neu aufgetretenen Symptomen, deren Beginn nicht mehr als $48 \mathrm{~h}$ zurückliegt (Ila, B).

Stabile, beschwerdefreie Patienten, bei denen die Symptome mehr als $48 \mathrm{~h}$ zurückliegen, profitieren nicht von einer Revaskularisation. Dies zeigte die große OATStudie (Occluded Artery Trial [127]). Patienten mit dieser Anamnese sollten wie Patienten mit einem chronisch verschlossenen Koronargefäß behandelt werden, und die Entscheidung zur elektiven Revaskularisation oder zu einem konservativen Procedere sollte unter Berücksichtigung von Vitalität, Ischämienachweis und subjektiven Beschwerden gefällt werden (s. Kap. 3) [46].

\subsubsection{Revaskularisationsstrategie beim STEMI}

Die PCl des Infarktgefäßes bei Patienten mit STEMI ist die Therapie der Wahl $(I, A)$. Dies liegt v. a. an der schnellen Durchführbarkeit und somit einer schnellen Reperfusion und der daraus resultierenden er- 
Hier steht eine Anzeige.

黑 Springer 


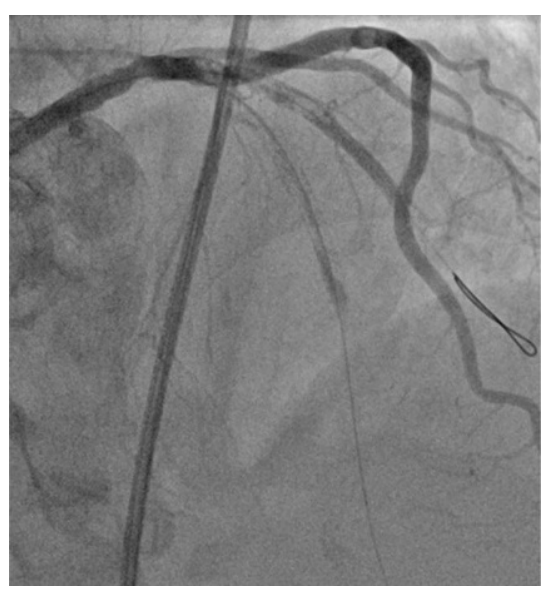

Abb. $19 \Delta$ LAD/D1 mit sehr hoher Thrombuslast

höhten Überlebenswahrscheinlichkeit bei erfolgreicher PCI [124].

Revaskularisation bei Mehrgefäß-KHK. In den letzten Jahren wurden viele Studien zur kompletten Revaskularisation bei Patienten mit STEMI und Mehrgefäßerkrankung durchgeführt. Diese verglichen [112, 128-130] eine komplette Revaskularisation während der Indexprozedur oder spätestens vor Entlassung gegen ein konservatives Procedere, bei dem nur die "culprit lesion" revaskularisiert wurde. In diesen Studien zeigte sich kein Vorteil hinsichtlich des Überlebens für die sofortige komplette Revaskularisation [131]. Allerdings zeigte sich eine signifikant geringere Rate erneuter Revaskularisationseingriffe für die Patienten, die im Indexaufenthalt alle relevanten Koronarstenosen behandelt bekamen. In der kürzlich publizierten COMPLETE-Studie konnte im Unterschied zu den oben genannten Studien erstmalig ein signifikanter Vorteil für die komplette Revaskularisation - entweder während der Indexprozedur, während des Indexaufenthaltes oder in einem erneuten elektiven Aufenthalt 45 Tage nach dem Indexereignis - im Vergleich zu einer alleinigen Therapie der "culprit lesion" bei Patienten mit STEMI belegt werden [131].

Aufgrund dieser Datenlage wird derzeit eine komplette Revaskularisation primär innerhalb des initialen Krankenhausaufenthalts empfohlen (Ila, A). Nach Meinung der Autoren und in Einbeziehung der Ergebnisse der COMPLETE-Studie kann eine erneute Revaskularisation jedoch auch im

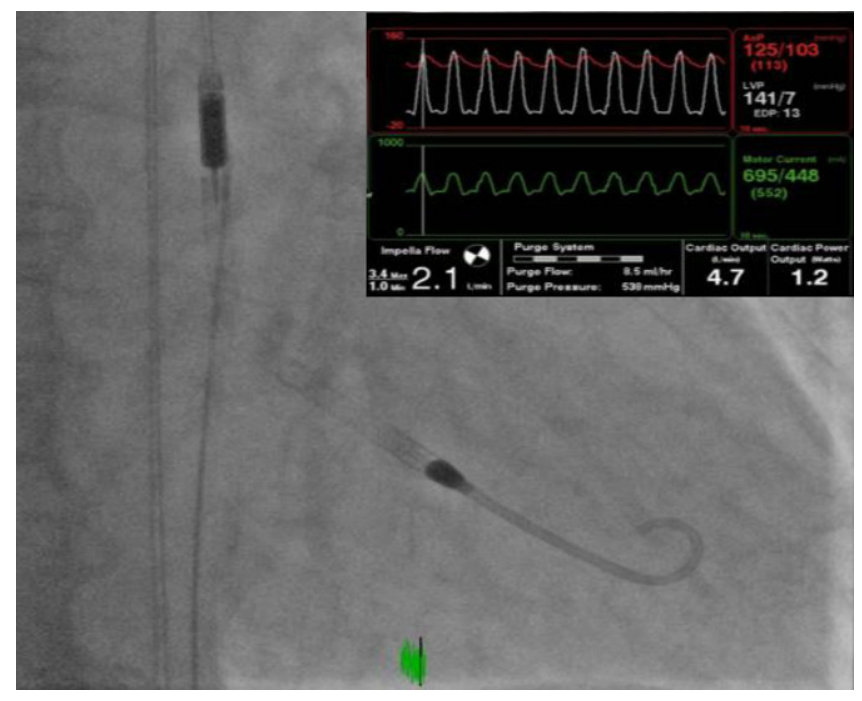

Abb. $20<$ Röntgenologische Darstellung einer Mikroaxialpumpe (IMPELLA) im linken Ventrikel sowie hämodynamisches Monitoring bei einer aktuellen Leistung von 2,1 I/min
Rahmen eines zeitnahen zweiten Krankenhausaufenthaltes geschehen.

Thrombusaspiration. Die 2 großen randomisierten Studien TASTE und TOTAL (insgesamt > 15.000 Patienten) zeigten keinen Vorteil der Thrombusaspiration hinsichtlich des jeweiligen primären Endpunkts (TASTE-Studie: Tod innerhalb von 30 Tagen, TOTAL-Studie: kardiovaskulärer Tod, erneuter Herzinfarkt, kardiogener Schock oder kardiale Dekompensation mit Ruhedyspnoe innerhalb von 180 Tagen) gegenüber der $\mathrm{PCl}$ ohne Thrombusaspiration $[132,133]$.Jedoch wiesen die Patienten, die eine Thrombusaspiration erhielten, in der TOTAL-Studie eine erhöhte Schlaganfallrate auf [134]. Die Thrombusaspiration sollte daher nicht mehr routinemäßig durchgeführt werden (Empfehlungsgrad III [6]). Aufgrund von Subgruppenanalysen und den Ergebnissen der TASTE-Studie, in denen doch gewisse Vorteile gesehen wurden [132, 134], kann die Thrombusaspiration jedoch bei hoher Thrombuslast und als „Rescue"-Maßnahme nach primär erfolgloser Ballondilatation (s. • Abb. 19) in Erwägung gezogen werden $[6,46]$.

\subsection{Indikation zur PCI bei ACS und kardiogenem Schock}

Ungefähr 3\% der Patienten mit einem NSTE-ACS und $9 \%$ mit einem STEMI entwickeln einen kardiogenen Schock [135, 136] meist infolge einer akuten Beeinträchtigung der LV-Funktion oder einer bereits vorbestehenden chronisch reduzier- ten LV-Funktion. Zudem können mechanische Komplikationen wie eine ischämiebedingte akute Mitralinsuffizienz einen kardiogenen Schock verursachen. Patienten mit einem ACS und einem begleitenden kardiogenen Schock haben eine hohe Letalität $[137,138]$ und sollten (s. Kap. 3) der sofortigen invasiven Diagnostik zugeführt werden $[6,45]$. Gerade bei Patienten mit STEMI und kardiogenem Schock verschlechtert sich die Prognose mit zunehmendem Intervall zwischen FMC und primärer $\mathrm{PCl}$ rapide. Jede $10 \mathrm{~min}$ Zeitverzögerung resultieren in einer absoluten Zunahme der Mortalität von 3,3\% [46, 124]. Dies kann teilweise auf Patienten mit NSTE-ACS und kardiogenem Schock übertragen werden - da auch hier häufig ein Gefäßverschluss dem Schockgeschehen zugrunde liegt [135]. Somit sollte insbesondere bei den Patienten im kardiogenen Schock und ACS (STEMI wie auch NSTE-ACS) auf einen schnellstmöglichen und daher direkten Transport ins Herzkatheterlabor und das Vermeiden jedweden Zeitverlustes (z.B. initiale Aufnahme auf eine Intensivstation) geachtet werden.

Im Falle einer Mehrgefäß-KHK sollte sich die grundsätzliche Revaskularisationsstrategie bei Patienten im kardiogenen Schock den Ergebnissen der CULPRIT SHOCK-Studie anpassen. Bei Patienten mit kompletter Revaskularisation trat der kombinierte Endpunkt aus Tod und Nierenersatzverfahren häufiger auf als bei den Patienten, bei denen lediglich die "culprit lesion" behandelt wurde [137]. Entsprechend sollte bei 


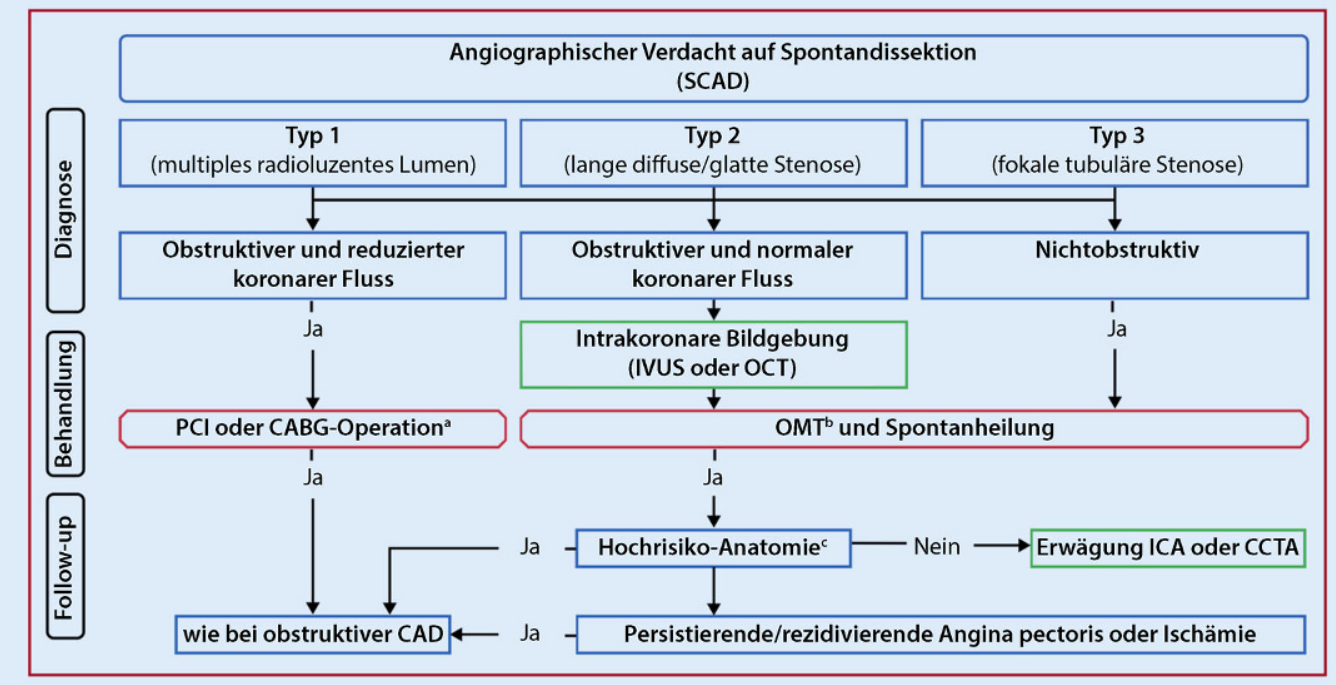

Abb. $21<$ Entscheidungsalgorithmus bei koronarer Spontandissektion. ${ }^{\text {a Aus- }}$ wahl der Revaskularisationsstrategie für Hochrisiko-Anatomien anhand lokaler Resourcen, ${ }^{\mathrm{b}}$ Betablocker wird empfohlen, eine Therapie mittels DAPT ist fraglich. ' Hauptstamm, proximale $L A D$, proximale RCX, RCA oder Multigefäß SCAD. (Mod. nach [45])
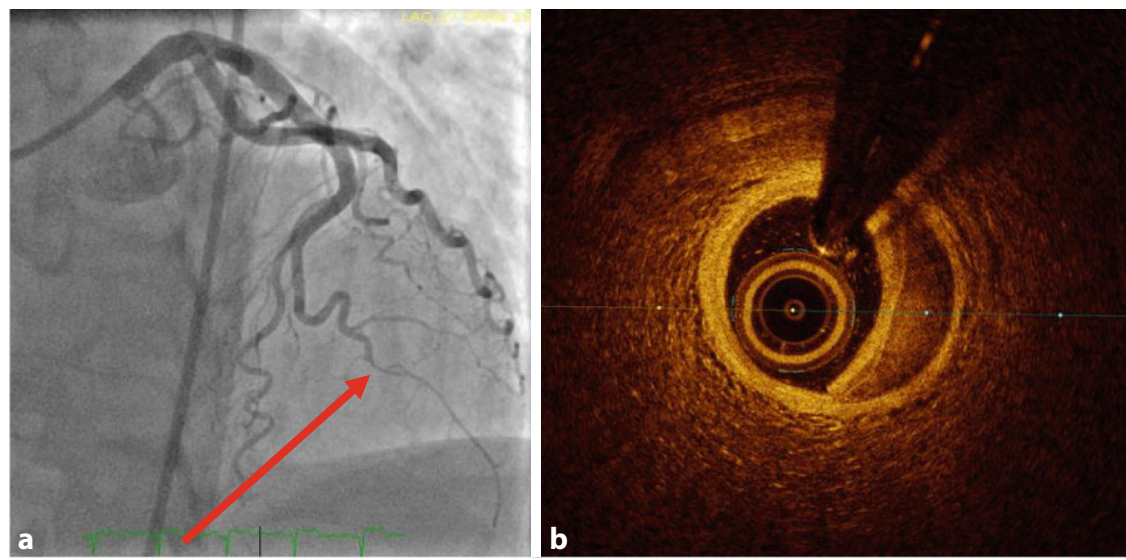

Abb. 22 \ Bild einer Spontandissektion: a angiographische Darstellung einer Dissektion (roter Pfeil), b Darstellung der Dissektion in der OCT-Untersuchung

diesen Patienten nur eine $\mathrm{PCl}$ der "culprit lesion" durchgeführt werden (I, B).

Eine Kreislaufunterstützung mittels intraventrikulärer Mikoraxialpumpe (IMPELLA, s. - Abb. 20) oder mittels extrakorporaler Kreislaufunterstützung (ECLS oder av-ECMO) ist eine Möglichkeit zur Behandlung eines kardiogenen Schocks. Sie wird jedoch aufgrund der geringen Datenlage lediglich mit einer IIb, C-Empfehlung bewertet [46, 104]. Die intraaortale Ballongegenpulsation (IABP) sollte aufgrund der Ergebnisse der IABP SHOCK-Studie nicht mehr routinemäßig eingesetzt werden (III, A) [138].

\subsection{Indikation zur PCI bei Spontandissektion}

Bei 1-4\% aller ACS liegt als Ursache eine koronare Spontandissektion zugrunde. Hierbei präsentieren sich ca. $70 \%$ als NSTEACS und $30 \%$ als STEMI. Am häufigsten sind junge Frauen betroffen [139-141]. Bei einem ACS infolge einer Spontandissektion sollte die Indikation zur PCI genau geprüft werden. Indikationen ergeben sich hierfür bei hämodynamischer Instabilität, bei anhaltenden Beschwerden, bei persistierenden EKG-Veränderungen, ventrikulären Rhythmusstörungen sowie in Abhängigkeit des angiographischen Erscheinungsbilds (s. - Abb. 21). Eine PCl einer Spontandissektion ist mit einem höheren interventionellen Risiko behaftet $[139,140]$. Ausgenommen der Patienten mit oben ge- nannten Kriterien, scheint eine rein konservative Therapie der Spontandissektion die zu präferierende Strategie zu sein [45].

Bei der Diagnostik kann eine intrakoronare Bildgebung hilfreich sein (- Abb. 22), jedoch besteht aufgrund der Vulnerabilität der Koronarien bei einer Spontandissektion hier ein erhöhtes Risiko von weiteren Komplikationen. Zur Verlaufskontrolle v. a. bei persistierenden Symptomen kann sich auch eine koronare CTA eignen [45].

\section{Medikamentenfreisetzende Koronarstents und medikamen- tenbeschichtete Ballonkatheter}

\subsection{Medikamentenfreisetzende Stents}

Der Einsatz von medikamentenfreisetzenden Stents ("drug-eluting stent" [DES]) konnte die Inzidenz der In-Stent-Restenose signifikant gegenüber dem unbeschichteten Stent (BMS) reduzieren. Die ersten DES (120-140 $\mu \mathrm{m}$ Strutdicke) waren mit Sirolimus bzw. Paclitaxel beschichtet und konnten das Risiko einer klinischen Restenose um $50-70 \%$ reduzieren, allerdings zeigte sich ein erhöhtes Risiko für Stentthrombosen im Vergleich zum BMS [6]. Diese Generation wurde durch die 2. Generation DES ersetzt. Diese Stents stellen eine deutliche Weiterentwicklung dar und sind mit einem deutlich biokompatibleren Polymer beschichtet (permanent oder biodegradierbar). Die Beschichtung ist 
Tab. 6 Maximale Expansionsmöglichkeiten der einzelnen Stentmodelle. Diese Angaben beziehen sich lediglich auf die mechanischen Eigenschaften ohne Berücksichtigung der Radialkraft und der Kinetik des freisetzenden Medikaments; eine ausführliche Darstellung ist dem Positionspapier der AGIK "Medikamentenfreisetzende Koronarstents/-scaffolds und medikamentenbeschichtete Ballonkatheter" im Auftrag der DGKzu entnehmen [142]

\begin{tabular}{|c|c|c|c|}
\hline Stentsystem & Hersteller & $\begin{array}{l}\text { Stentgröße bei Nomi- } \\
\text { naldruck }(\mathrm{mm})\end{array}$ & $\begin{array}{l}\text { Potenzieller Lumendiameter } \\
\text { nach Überexpansion ( } \mathrm{mm} \text { ) }\end{array}$ \\
\hline \multirow{4}{*}{$\begin{array}{l}\text { Promus Ele- } \\
\text { ment }\end{array}$} & \multirow{4}{*}{$\begin{array}{l}\text { Boston Sci- } \\
\text { entific }\end{array}$} & 2,25 & 3,0 \\
\hline & & $\mathrm{Ab} \mathrm{2,5}$ & 3,8 \\
\hline & & $\mathrm{Ab} 3,0$ & 4,4 \\
\hline & & $\mathrm{Ab} 4,0$ & 5,7 \\
\hline \multirow[t]{2}{*}{ Xience } & \multirow{2}{*}{$\begin{array}{l}\text { Abbott } \\
\text { Vascular }\end{array}$} & Ab 2,25 & 4,4 \\
\hline & & $\mathrm{Ab} 3,5$ & 5,6 \\
\hline \multirow{2}{*}{$\begin{array}{l}\text { Resolute Inte- } \\
\text { grity }\end{array}$} & \multirow[t]{2}{*}{ Medtronic } & Ab 2,25 & 4,9 \\
\hline & & $\mathrm{Ab} 3,0$ & 5,4 \\
\hline \multirow{2}{*}{$\begin{array}{l}\text { Biomatrix } \\
\text { Neoflex }\end{array}$} & \multirow[t]{2}{*}{ Biosensors } & Ab 2,25 & 4,6 \\
\hline & & $\mathrm{Ab} 3,5$ & 5,9 \\
\hline \multirow[t]{3}{*}{ Synergy } & \multirow{3}{*}{$\begin{array}{l}\text { Boston Sci- } \\
\text { entific }\end{array}$} & Ab 2,25 & 3,6 \\
\hline & & $\mathrm{Ab} 3,5$ & 4,2 \\
\hline & & $\mathrm{Ab} 4,0$ & 5,7 \\
\hline \multirow[t]{2}{*}{ Orsiro } & \multirow[t]{2}{*}{ Biotronik } & Bis 3,0 & 4,0 \\
\hline & & $\mathrm{Ab} 3,5$ & 5,3 \\
\hline \multirow[t]{2}{*}{ Ultimaster } & \multirow[t]{2}{*}{ Terumo } & Bis 3,0 & 4,3 \\
\hline & & $\mathrm{Ab} 4,5$ & 5,8 \\
\hline \multirow[t]{4}{*}{ Resolute Onyx } & \multirow[t]{4}{*}{ Medtronic } & Bis 2,5 & 3,3 \\
\hline & & $\mathrm{Ab} 3,0$ & 4,4 \\
\hline & & $\mathrm{Ab} 4,0$ & 5,6 \\
\hline & & $\mathrm{Ab} 5,0$ & 6,0 \\
\hline
\end{tabular}

ausschließlich mit einem Sirolimus-Analogon versehen. Der Stent selbst besteht aus Edelstahl, Kobaltchrom oder Platinchrom. Die DES-Stents der 2. Generation haben eine höhere Wirksamkeit und Sicherheit im Vergleich zu den beiden DES der ersten Generation und den BMS mit einem mittel- bis langfristig ähnlichen Risiko für Tod oder Myokardinfarkt im Vergleich zu den BMS. Das Risiko für eine Stentthrombose ist allerdings bei den DES-Stents der 2. Generation deutlich geringer als bei den BMS-Stents. Diese Beobachtungen wurden in einer kürzlich durchgeführten Studie bestätigt, in der Patienten im Alter von 75 Jahren oder älter eingeschlossen wurden und die eine Überlegenheit des kombinierten Endpunktes (zusammengesetzt aus Gesamtmortalität, Myokardinfarkt, Schlaganfall oder Ischämie-bedingter Revaskularisierung der Zielläsion) in der mit DES versorgten Patientengruppe im Vergleich zu BMS zeigte. Es zeigte gleichermaßen kein Hinweis auf einen Unterschied zwischen DES und BMS Kohorte hinsichtlich des
Stentthromboserisikos nach ungeplantem Absetzen der DAPT-Therapie [142].

Nach den Empfehlungen der European Society of Cardiology sollten DES der neuen Generation für den Routineeinsatz den BMS vorgezogen werden (I, A). Die Vorteile der DES der neuen Generation bleiben auch bei Patienten mit Vorhofflimmern bestehen. Besonders Patienten mit Diabetes mellitus profitieren vom Einsatz der DES der neuen Generation.

In mehreren Studien konnte für DES mit biodegradierbarem Polymer kein Vorteil gegenüber dem dauerhaften Polymer dokumentiert werden, sodass derzeit keine Empfehlung hinsichtlich einer Bevorzugung dieser Stentkategorie besteht.

Die Kenntnis der Expansionseigenschaften der einzelnen Stents ist für die Behandlung großkalibriger Gefäße (Hauptstamm) sowie von Regionen mit unterschiedlichen Gefäßdiametern („tapered vessel“) von großer Bedeutung (- Tab.6). Es sei aber darauf hingewiesen, dass diese Angaben nicht den Empfehlungen der Hersteller entsprechen und ggf. hierdurch Limitationen bezüglich Radialkraft oder Integrität des Polymers auftreten können.

\subsection{Medikamentenbeschichtete Ballons}

Aufgrund verschiedener Limitationen der DES in bestimmten Läsionstypen (z. B. kleine Gefäße, In-Stent Restenosen, Bifurkationen etc.) wurden medikamentenbeschichtete Ballons (DCB) als Alternative untersucht.

Der DCB zeigt beim Einsatz in De-novoStenosen heterogene Daten. Untersucht wurde insbesondere die Kombination $\mathrm{DCB}+\mathrm{BMS}$ vs. DES. In keiner Studie konnte eine Überlegenheit zu einem DES der zweiten Generation gezeigt werden.

Bei In-Stent-Restenosen (ISR) wird derzeit die Behandlung mit einem DCB empfohlen $(I, A)$, wobei ein adäquater Vergleich mit DES der zweiten Generation aussteht [143]. Ob die zugrunde liegende Ursache (Neointima-Hyperproliferation oder Neoatherosklerose) der ISR Einfluss auf die Therapie nimmt, ist derzeit unbeantwortet [6]. In $\square$ Tab. 7 sind die in Deutschland zugelassenen DEB dargestellt.

\section{Antithrombotische Behand- lungsstrategie bei $\mathrm{PCl}$}

\subsection{Chronische KHK}

\subsubsection{Thrombozytenaggregations- hemmer}

Der klinische Nutzen einer Monotherapie mit niedrig dosiertem ASS ist nur in einer placebokontrollierten Studie bei Patienten mit stabiler Angina und Ballondilatation untersucht worden (I, B) [145]. Gleichwohl wird angenommen, dass der Nutzen einer ASS-Behandlung in ähnlicher Weise für andere koronare Interventionen und klinische Situationen gegeben ist $(\mathrm{I}, \mathrm{C})[146$, 147].

Patienten mit ASS-Unverträglichkeit sollten Clopidogrel zur Hemmung des P2Y 12 -Rezeptors erhalten. Bei Vorliegen einer ASS-Allergie kann eine kurzzeitige Desensibilisierung erfolgen (s. - Tab. 8) Eine normale Thrombozytenfunktion wird ca. 7 Tage nach der letzten ASS-Dosis wieder erreicht. Eine höhere Dosierung 
Tab. 7 In Deutschland zugelassene medikamentenbeschichtete Ballonkatheter [144]

\begin{tabular}{|c|c|c|c|c|c|}
\hline Ballonsystem & Hersteller & Zusatz zur Beschichtung & Wirksubstanz & $\begin{array}{l}\text { Angiographische End- } \\
\text { punktstudien }\end{array}$ & $\begin{array}{l}\text { Ausgewählte Lite- } \\
\text { ratur }\end{array}$ \\
\hline SeQuent Please & B. Braun & Kontrastmittel (lopromid) & $\begin{array}{l}\text { Paclitaxel } \\
3 \mu \mathrm{g} / \mathrm{mm}^{2}\end{array}$ & ++ ISR, DN, Bif, SVD & $\begin{array}{l}{[35,40,41,43,44,} \\
48-53]\end{array}$ \\
\hline Pantera Lux & Biotronik & Butyryl-trihexyl-Zitrat & $\begin{array}{l}\text { Paclitaxel } \\
3 \mu \mathrm{g} / \mathrm{mm}^{2}\end{array}$ & $(+)$ ISR & {$[54-56]$} \\
\hline In.Pact Falcon & Medtronic & Harnstoff & $\begin{array}{l}\text { Paclitaxel } \\
3 \mu \mathrm{g} / \mathrm{mm}^{2}\end{array}$ & (+) Bif, DN & {$[57,58,60]$} \\
\hline Cotavance & $\begin{array}{l}\text { Medrad Interven- } \\
\text { tional }\end{array}$ & Kontrastmittel (Ultravist) & $\begin{array}{l}\text { Paclitaxel } \\
3 \mu \mathrm{g} / \mathrm{mm}^{2}\end{array}$ & - & N.a. \\
\hline Dior II & Eurocor & Schellack & $\begin{array}{l}\text { Paclitaxel } \\
3 \mu \mathrm{g} / \mathrm{mm}^{2}\end{array}$ & (+) Bif, DN & {$[62,65,66]$} \\
\hline Protege & Blue Medical & Butyryl-trihexyl-Zitrat & $\begin{array}{l}\text { Paclitaxel } \\
3 \mu \mathrm{g} / \mathrm{mm}^{2}\end{array}$ & - & N.a. \\
\hline Elutax & Aachen Resonance & Kein Exzipient & $\begin{array}{l}\text { Paclitaxel } \\
2 \mu \mathrm{g} / \mathrm{mm}^{2}\end{array}$ & $(+)$ ISR & [37] \\
\hline Restore DCB & Cardionovum & $\begin{array}{l}\text { Kein Exzipient } \\
\text { Paclitaxel als Nanokristalle }\end{array}$ & $\begin{array}{l}\text { Paclitaxel } \\
3 \mu \mathrm{g} / \mathrm{mm}^{2}\end{array}$ & $(+)$ ISR & [67] \\
\hline Danubio & Minvasys & Butyryl-trihexyl-Zitrat & $\begin{array}{l}\text { Paclitaxel } \\
2,5 \mu \mathrm{g} / \mathrm{mm}^{2}\end{array}$ & (+) Bif & [68] \\
\hline Agent & Boston & Citratester & $\begin{array}{l}\text { Paclitaxel } \\
2 \mu \mathrm{g} / \mathrm{mm}^{2}\end{array}$ & - & N.a. \\
\hline Biostream & Biosensors & Shellac & $\begin{array}{l}\text { Paclitaxel } \\
3 \mu \mathrm{g} / \mathrm{mm}^{2}\end{array}$ & - & N.a. \\
\hline Moxy & Lutonix & Polysorbate & $\begin{array}{l}\text { Paclitaxel } \\
3 \mu \mathrm{g} / \mathrm{mm}^{2}\end{array}$ & (+) DN & [70] \\
\hline
\end{tabular}

Tab. 8 Schema der ASS-Desensibilisierung; eine Überwachung möglicher Symptome muss jederzeit gewährleistet sein, bei Patienten mit einer anaphylaktischen Reaktion in der Vorgeschichte muss in den ersten 30 min eine Überwachung erfolgen; im Fall einer anaphylaktischen Reaktion muss eine individuelle Therapie der Anaphylaxie umgehend eingeleitet werden; im Fall einer nicht applizierten Dosis muss das Protokoll von Anfang an wiederholt werden

\begin{tabular}{|l|l|}
\hline Zeitpunkt & $\begin{array}{l}\text { ASS-Dosis in } \text { mg } \\
\text { (i.v., p.o., p.ms.) }\end{array}$ \\
\hline 0 min & 0,1 \\
\hline $30 \mathrm{~min}$ & 0,2 \\
\hline $60 \mathrm{~min}$ & 0,4 \\
\hline $90 \mathrm{~min}$ & 0,8 \\
\hline $120 \mathrm{~min}$ & 1,6 \\
\hline $150 \mathrm{~min}$ & 3,2 \\
\hline $180 \mathrm{~min}$ & 6 \\
\hline $210 \mathrm{~min}$ & 10 \\
\hline $240 \mathrm{~min}$ & 20 \\
\hline $270 \mathrm{~min}$ & 40 \\
\hline $300 \mathrm{~min}$ & 80 \\
\hline $330 \mathrm{~min}$ & 160 \\
\hline $360 \mathrm{~min}$ & 240 \\
\hline
\end{tabular}

von ASS (>100 mg) erhöht nur das Blutungsrisiko.

Ohne Loading-Dosis wird bei einer Clopidogrel-Dosis von $75 \mathrm{mg} / \mathrm{Tag}$ nach ca. 4 bis 7 Tagen ein Steady-State erreicht. Bei nicht vorbehandelten Patienten wird nach $300 \mathrm{mg}$ oral eine relevante Wirkung nach $6 \mathrm{~h}$, nach $600 \mathrm{mg}$ oral eine Wirkung nach ca. $2 \mathrm{~h}$ erreicht. Eine normale Thrombozytenfunktion stellt sich ca. 7 Tage nach Absetzen wieder ein.

Üblicherweise wird heute nach jeder Koronarintervention in der Regel für 6 Monate, mindestens jedoch für 4 Wochen, eine duale Plättchenhemmung, d.h. ASS plus Clopidogrel, verordnet (s. - Tab. 9).

Eine routinemäßige duale Vorbehandlung vor einer diagnostischen Koronarangiographie wird nicht empfohlen, da eine klinische Überlegenheit nicht belegt ist. Allerdings sollte dies bei Patienten mit hoher Wahrscheinlichkeit für eine $\mathrm{PCl}$ erwogen werden, z.B. bei einem entsprechendem CT-Befund. Jede geplante PCI (z. B. "staged procedure") sollte unter dualer Plättchenhemmung erfolgen, außer bei einer geplanten Rekanalisation mit hohem Perforationsrisiko (I, C).
Alternativ zu Clopidogrel kann im Einzelfall bei elektiver $\mathrm{PCl}$ auch Prasugrel oder Ticagrelor bei bestimmten Hochrisikoeingriffen (z. B. Hauptstamm, lange Stentlängen) oder bei früherer Stentthrombose unter Clopidogrel erwogen werden.

Nach Absetzen der dualen Plättchenhemmung ist eine lebenslange Therapie mit ASS $100 \mathrm{mg} / \mathrm{Tag}$, bei Unverträglichkeit mit Clopidogrel $75 \mathrm{mg} / \mathrm{Tag}$ angezeigt. Dies sollte nach Möglichkeit auch bei operativen Eingriffen (außer beim Gehirn und Zentralnervensystem) nicht abgesetzt werden.

Cangrelor ist ein reversibler, kurz wirksamer $\mathrm{P}_{2} \mathrm{Y}_{12}$-Rezeptorblocker, der i.v. verabreicht werden kann und in den CHAMPIONStudien untersucht wurde. Nach einer Metaanalyse werden allerdings die Vorteile bei ischämischen Endpunkten durch Blutungskomplikationen egalisiert [148]. Bei einer Vorbehandlung mit Clopidogrel ist kein wesentlicher Vorteil mehr nachweisbar [149]. Trotzdem ist es bei bisher nicht vorbehandelten Patienten mit niedrigem Blutungsrisiko eine gute Therapieoption, um die Rate an postinterventionellen Stentthrombosen intra- und unmittelbar postinterventionell zu senken. 
Tab. 9 Empfehlungen zur antithrombotischen Therapie bei stabiler KHKnach PCI [6] Empfehlung zur antithrombotischen Therapie bei Myokardrevaskularisierung

Vor der Prozedur

Therapie mit $600 \mathrm{mg}$ Clopidogrel ist bei elektiven PCl empfohlen, wenn die Anatomie der Koronarien bekannt ist und die Entscheidung zur $\mathrm{PCl}$ getroffen wurde

Bei hoher Wahrscheinlichkeit für eine $\mathrm{PCl}$ sollte Clopidogrel vor Beginn verabreicht werden

Im Fall einer eingenommenen Erhaltungstherapie mit $75 \mathrm{mg}$ Clopidogrel sollte eine erneute Gabe von $600 \mathrm{mg}$ Clopidogrel nach

Indikationsstellung für die $\mathrm{PCl}$ erfolgen

Periprozedurale Therapie

ASS ist vor elektivem Stenting indiziert

Eine Loading-Dosis mit 150-300 mg p.o. oder 75-250 mg i.v. ist bei Patienten empfohlen, die keine ASS-Vormedikation erhalten

Clopidogrel (600 mg Loading-Dosis, $75 \mathrm{mg}$ täglich als Erhaltungsdosis) ist bei elektivem Stenting empfohlen

Glykoprotein-Ila/Illb-Antagonisten sollten nur im Notfall in Erwägung gezogen werden

Prasugrel oder Ticagrelor sollten bei speziellen hoch risikohaften Situationen (z. B. frühere Stentthrombose, Stenting des Haupt-

stamms) bei elektivem Stenting in Erwägung gezogen werden

Unfraktioniertes Heparin ist indiziert als Standardantikoagulation $(70-100 \mathrm{mg} / \mathrm{kg}$ i.v.)

Bilivarudin (0,75 mg/kg i.v.-Bolus, gefolgt von einer Erhaltungsdosis mit 1,75 mg/kg/h bis zu $4 \mathrm{~h})$ ist indiziert für den Fall einer Heparin-indizierten Thrombozytopenie

Enoxaparin (0,5 mg/kg i.v.) sollte als Alternative in Erwägung gezogen werden

Cangrelor sollte bei P2 $\mathrm{Y}_{12}$-naiven Patienten während einer elektiven $\mathrm{PCl}$ in Erwägung gezogen werden

Postprozedurale Therapie und Erhaltungstherapie

Lebenslange antithrombozytäre Therapie, meist mit ASS, wird empfohlen

Aufklärung der Patienten über die Notwendigkeit der regelmäßigen Einnahme der Therapie wird empfohlen

Bei Patienten mit stabiler KHK und Stentimplantation wird eine 6-monatige Therapie, unabhängig vom Stenttyp, mittels DAPT (Clopidogrel und meistens ASS) empfohlen

Bei Patienten mit stabiler KHK und Implantation von bioresorbierbaren Gefäßstützen wird eine mindestens 12-monatige Therapie mittels DAPT empfohlen. Eine Verlängerung bis zum vermuteten Zeitpunkt der Resorption kann unter Abschätzung der individuellen Blutungs- und Ischämierisiken in Erwägung gezogen werden

Bei Patienten mit stabiler KHK und PCI mittels DCB, DAPT sollte für 6 Monate in Erwähnung gezogen werden

Bei Patienten mit stabiler KHK sollte eine 3-monatige DAPT bei hohem Risiko für Blutungen (z. B.: PREC6E-DAPT $\geq 25$ ) in Erwägung gezogen werden

Bei Patienten mit stabiler KHK, die eine DAPT tolerieren, die ein niedriges Blutungsrisiko und ein hohes Risiko für Thrombosen aufweisen, kann eine DAPT mit Clopidogrel für $>6$ Monate bis hin zu 30 Monaten in Erwägung gezogen werden

Bei Patienten mit sKHK, bei denen 3 Monate DAPT ein Sicherheitsrisiko darstellen, kann DAPT über 1 Monat erwogen werden

Klasse $\quad$ Level

I $\quad$ A

A Ilb

C

C

Ilb

\begin{tabular}{l|l}
\hline & $A$ \\
\hline I & C
\end{tabular}

A

Ila $\mathrm{C}$

Ilb C

\begin{tabular}{l|l}
\hline & B
\end{tabular}

$\begin{array}{ll}1 & \text { B } \\ \text { I } & \text { C }\end{array}$

Ila $\quad$ B

Ilb A

A


Tab. 10 Periprozedurale und postprozedurale antithrombotische Therapie bei Patienten mit primärer PCI[6]

\begin{tabular}{|c|c|c|}
\hline Empfehlung & Klasse & Level \\
\hline \multicolumn{3}{|l|}{ Antithrombozytäre Therapie } \\
\hline $\begin{array}{l}\text { Ein potenter P2 } \mathrm{Y}_{12} \text {-Inhibitor (Prasugrel oder Ticagrelor) oder, wenn nicht verfügbar oder kontraindiziert, Clopidogrel wird vor } \\
\text { (oder spätestens während) der Prozedur empfohlen zusammen mit einer 12-monatigen Erhaltungsdosis unter Beachtung von } \\
\text { Kontraindikationen und dem Risiko von möglichen Blutungen }\end{array}$ & I & A \\
\hline ASS (p.o oder i.v.) wird frühestmöglich empfohlen, wenn keine Kontraindikationen bestehen & I & A \\
\hline $\begin{array}{l}\text { GPIla/Illb-Inhibitoren sollten im Notfall in Betrachtung gezogen werden, wenn Evidenz für "no-flow" oder eine thrombotische } \\
\text { Komplikation besteht }\end{array}$ & Ila & $\mathrm{C}$ \\
\hline Cangrelor sollte in Betrachtung gezogen werden, wenn keine $\mathrm{P}_{2} \mathrm{Y}_{12}$-Inhibitoren verabreicht wurden & $\mathrm{llb}$ & A \\
\hline \multicolumn{3}{|l|}{ Antikoagulation } \\
\hline Antikoagulation wird für alle Patienten während einer primären $\mathrm{PCl}$ empfohlen & I & A \\
\hline Der routinemäßige Einsatz von UFH wird empfohlen & I & C \\
\hline Routinegebrauch von Enoxaparin i.v. sollte in Betracht gezogen werden & Ila & B \\
\hline Gebrauch von Bilivarudin i.v. kann als Alternative zu unfraktioniertem He & $\mathrm{Ilb}$ & A \\
\hline
\end{tabular}

\subsubsection{Orale Antikoagulation und} Thrombozytenaggregationshemmung

Grundsätzlich sollte bei Patienten mit chronischem Koronarsyndrom nach $\mathrm{PCl}$ und der Notwendigkeit zur oralen Antikoagulation ein NOAC (vorher DOAK) verwendet werden, sofern keine Kontraindikationen hierfür bestehen (Apixaban $5 \mathrm{mg}$ 2-mal täglich, Dabigatran $150 \mathrm{mg}$ 2-mal täglich, Edoxaban $60 \mathrm{mg}$ täglich, Rivaroxaban $20 \mathrm{mg}$ täglich) $(I, C)$. Bei der Verwendung von Rivaroxaban und Dabigatran sollte bei Vorliegen eines hohen Blutungsrisikos die Reduktion der Dosis auf 1-mal täglich $15 \mathrm{mg}$ bzw. 2-mal täglich $110 \mathrm{mg}$ erwogen werden (Ila, B). Bei Patienten mit erhöhtem Blutungsrisiko und nach unkomplizierter $\mathrm{PCl}$ sollte die Gabe von ASS in Kombination mit Clopidogrel und oraler Antikoagulation nicht länger als 1 Woche erfolgen (Ila, B).

\subsection{Akutes Koronarsyndrom}

Die meisten Patienten sind bereits im Notarztwagen bzw. in der Notaufnahme mit ASS und UFH vorbehandelt worden, bevor sie in das Katheterlabor kommen.

\subsubsection{Thrombozytenaggregations- hemmer}

Den Eckstein der Thrombozytenaggregationshemmung bildet in jedem Fall ASS $>75 \mathrm{mg} / \mathrm{Tag}$ mit Inhibition von Thromboxan $\mathrm{A}_{2}$ [45].

Entsprechend den Ergebnissen der PLATO- und der TRITON-TIMI 38-Studie wird Ticagrelor bzw. Prasugrel dem schwä- cher wirksamen Clopidogrel vorgezogen, da die Rate ischämischer Ereignisse niedriger ist [153, 154]. Clopidogrel sollte nur gegeben werden, wenn gegen die anderen beiden $\mathrm{P}_{2} \mathrm{Y}_{12}$-Hemmer eine Kontraindikation besteht.

Eine prästationäre Vorbehandlung mit Ticagrelor bei STEMI hat sich gegenüber einer intrahospitalen Gabe als nicht überlegen erwiesen. Clopidogrel sollte im Notarztwagen nicht mehr gegeben werden, da die plättchenhemmende Wirkung zu spät einsetzt, sodass im Katheterlabor noch keine wirksame Hemmung erwartet werden kann [155]. Bei Patienten mit NSTEMI zeigte sich unter Vorbehandlung mit Prasugrel kein Vorteil, aber eine erhöhte Rate an Blutungskomplikationen [156]. Da die koronare Anatomie im prähospitalen Setting nicht bekannt ist, wird eine entsprechende Vorbehandlung bei NSTE-ACS in den aktuellen Leitlinien grundsätzlich nicht empfohlen [45].

In einer kürzlich publizierten randomisierten Studie konnte ein signifikanter Vorteil bei Patienten mit ACS hinsichtlich des kombinierten Endpunktes aus Tod, Myokardinfarktinfarkt nach 12 Monaten zugunsten von Prasugrel im Vergleich zu Ticagrelor nachgewiesen werden. Die Sicherheitsendpunkte bezüglich Blutungen waren in beiden Gruppen vergleichbar. Im Unterschied zur Ticagrelor-Gabe erfolgte bei Patienten mit NSTE-ACS die Gabe der Loading-Dosis von Prasugrel erst nach der Durchführung der Koronarangiographie. Bei den Patienten mit STEMI wurden beide Substanzen unmittelbar vor der Koronarangiographieverabreicht. Weiterhin giltes, die Kontraindikationen von Prasugrel (früherer Apoplex/TIA, > 75 Jahre Lebensalter, Körpergewicht $<60 \mathrm{~kg}$ ) zu beachten [157].

Die wirksamste Thrombozytenhemmung kann mit Glykoprotein-Ilb/llaRezeptorantagonisten (Tirofiban, Eptifibatide, Abciximab) (GPA) erzielt werden, führt allerdings auch zu mehr Blutungskomplikationen. Die prähospitale Gabe von GPIlb/llla-Antagonisten (Tirofiban und Abciximab) hat sich im Vergleich zur Gabe im Katheterlabor ohne überzeugenden Vorteil erwiesen [158]. Im Katheterlabor ist unter dem derzeitigen Therapieregime mit schnell wirksamen P2 $Y_{12}$-Blockern ein Einsatz von GPA beim STEMI nicht routinemäßig notwendig und nur speziellen Situationen bei STEMI und NSTEMI vorbehalten (großer Thrombus, "slow flow", „no-reflow“, „bail-out”) (III, C). Abciximab als GPA ist seit dem 15.12.2018 produktionsbedingt nicht mehr lieferbar. Eine Übersicht über Dosierungen der antithrombozytären Therapie bzw. Antikoagulation während oder nach myokardialer Revaskularisierung ist in DTab. 11 abgebildet.

\subsubsection{Antikoagulation}

Ebenso wie bei elektiver $\mathrm{PCl}$ stehen die Antikoagulanzien UFH, Enoxaparin und Bivalirudin zur Auswahl.

Für UFH gibt es keine randomisierte Studie, aber die langjährige Erfahrung spricht für die Wirksamkeit. Es gibt keine belastbaren Daten, routinemäßig die ACT zu messen, obwohl dieses bei längeren Prozeduren oder z. B. Thrombusbildung an Drähten angezeigt sein kann. 


\section{Curriculum}

Tab. 11 Dosierungen der antithrombozytären Therapie bzw. Antikoagulation während oder nach myokardialer Revaskularisierung [6] Antithrombozytäre Therapie

\begin{tabular}{|c|c|}
\hline ASS & Loading-Dosis mit 150-300 mg p.o. oder 75-250 mg i.v., falls oral nicht möglich, gefolgt von $75-100 \mathrm{mg} / \mathrm{Tag}$ \\
\hline Clopidogrel & Loading-Dosis mit 300-600 mg p.o., gefolgt von einer Erhaltungsdosis von $75 \mathrm{mg} / \mathrm{Tag}$ \\
\hline Prasugrel & $\begin{array}{l}\text { Loading-Dosis mit } 60 \mathrm{mg} \text { p.o., gefolgt von einer Erhaltungsdosis von } 10 \mathrm{mg} / \mathrm{Tag} \\
\text { Bei Patienten mit einem Körpergewicht } \leq 60 \mathrm{~kg} \text { wird eine angepasste Erhaltungsdosis von } 5 \mathrm{mg} / \mathrm{Tag} \text { empfohlen } \\
\text { Bei älteren Patienten ( } \geq 75 \text { Jahre) sollte Prasugrel mit Vorsicht eingesetzt werden, und eine Erhaltungsdosis von } \\
5 \mathrm{mg} / \text { Tag ist empfohlen } \\
\text { Ein vorangegangener Schlaganfall ist eine Kontraindikation }\end{array}$ \\
\hline Ticagrelor & Loading-Dosis mit $180 \mathrm{mg}$ p.o., gefolgt von einer Erhaltungsdosis mit 2-mal täglich $90 \mathrm{mg}$ \\
\hline Cangrelor & $\begin{array}{l}\text { Bolus von } 30 \mu \mathrm{g} / \mathrm{kg} \text { i.v., gefolgt von einer Erhaltungsdosis mit } 4 \mu \mathrm{g} / \mathrm{kg} / \mathrm{min} \text { für die Dauer der Prozedur, mindestens aber } \\
2 \mathrm{~h} \text { lang }\end{array}$ \\
\hline Abciximab & Bolus von $0,25 \mathrm{mg} / \mathrm{kg}$ i.v. und $0,125 \mu \mathrm{g} / \mathrm{kg} / \mathrm{min}$ i.v. (maximal $10 \mu \mathrm{g} / \mathrm{min}$ ) über $12 \mathrm{~h}$ \\
\hline Eptifibatide & Doppelter Bolus von $180 \mu \mathrm{g} / \mathrm{kg}$ i.v. (im 10-min-Intervall), gefolgt von $2,0 \mu \mathrm{g} / \mathrm{kg} / \mathrm{min}$ für bis zu $18 \mathrm{~h}$ \\
\hline Tirofiban & 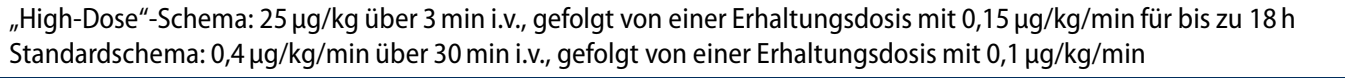 \\
\hline \multicolumn{2}{|c|}{ Parenterale Antikoagulanzien } \\
\hline UFH & $\begin{array}{l}\text { 70-100 IE/kg i.v.-Bolus, wenn ein GPIllb/Illa-Inhibitor nicht geplant ist } \\
\text { Oder 50-70 IE/kg i.v.-Bolus zusammen mit GPIlb/Illa-Inhibitoren }\end{array}$ \\
\hline Enoxaparin & $0,5 \mathrm{mg} / \mathrm{kg}$ i.v.-Bolus \\
\hline Bilivarudin & $0,75 \mathrm{mg} / \mathrm{kg}$ i.v.-Bolus, gefolgt von $1,75 \mathrm{mg} / \mathrm{kg} / \mathrm{h}$ i.v. für bis zu $4 \mathrm{~h}$ nach der Prozedur \\
\hline Fondaparinux & $2,5 \mathrm{mg} / \mathrm{Tag}$ s.c. (nur vor der PCl) \\
\hline \multicolumn{2}{|c|}{ Orale Antikoagulanzien nach PCI } \\
\hline Vitamin-K-Antagonisten & Dosis nach INR \\
\hline Apixaban & Erhaltungsdosis $5 \mathrm{mg}$ 2-mal täglich \\
\hline Dabigatran & Erhaltungsdosis $150 \mathrm{mg}$ 2-mal täglich \\
\hline Edoxaban & Erhaltungsdosis $60 \mathrm{mg}$ täglich \\
\hline Rivaroxaban & Erhaltungsdosis $20 \mathrm{mg}$ täglich \\
\hline
\end{tabular}

Enoxaparin ist eine gute Alternative zu UFH, bei allerdings etwas widersprüchlicher Studienlage [159, 160]. Bivalirudin wurde in zahlreichen großen Studien bei STEMI-Patienten mit oder ohne GPIIb/IIlaAntagonisten untersucht [161]. Die Ergebnisse der ACUITY- und MATRIX-Studie zeigten keine Unterlegenheit von Bivalirudin im ACS bei signifikant weniger Blutungsereignissen. Im Gegensatz hierzu steht die HEAT-PPCI-Studie, in der bei der Anwendung von Bivalirudin die Rate an akuten Stentthrombosen gegenüber Heparin signifikant erhöht war [162-164].

In der HEAT-PCI-Studie konnte bei ACSPatienten kein Vorteil von Blutungsraten bei erhöhter Rate an Stentthrombosen gezeigt werden [165]. Nach erfolgreicher Prozedur ist keine weitere Antikoagulation erforderlich, sofern dies nicht aus anderen Gründen indiziert ist (z. B. Vorhofflimmern, LV-Thrombus).

\subsubsection{Orale Antikoagulation und Thrombozytenaggregationshem- mung}

Bei Patienten mit Indikation zur oralen Antikoagulation sind besondere Dosierungsschemata zu beachten. Hier wird auf die Empfehlung der europäischen Leitlinien hingewiesen (siehe - Abb. 23) [47].

Die duale Plättchenhemmung in Kombination mit einer oralen Antikoagulation ist zu verkürzen (ASS nur maximal 4 Wochen), um Blutungskomplikationen zu vermeiden (Ila, B). Clopidogrel ist dabei der standardmäßig einzusetzende $\mathrm{P}_{2} \mathrm{Y}_{12}$-Blocker (6 bis 12 Monate). Prasugrel oder Ticagrelor in Kombination mit oraler Antikoagulation können als Alternative zur Triple-Therapie nach strenger Indikation (z. B. sehr hohes Risiko für Stentthrombose) zum Einsatz kommen (II, C). Sofern keine anderen Gründe vorliegen (z. B. Stentthrombose), kann die Plättchenhemmung nach 1 Jahr vollständig beendet werden (Ila, B) (s. - Tab. 10).

Zur Antikoagulation ist ein VitaminK-Antagonist (z. B. Phenprocoumon) wei- terhin indiziert bei Patienten mit mechanischem Herzklappenersatz, $\geq$ mittelschwerer Mitralstenose mit Vorhofflimmern und schwerer Niereninsuffizienz (GFR $<15 \mathrm{ml}$ ) (s. - Abb. 24).

In 4 Studien wurden die Vorteile der NOACs im Vergleich zu Phenprocoumon im Hinblick auf Blutungskomplikationen belegt. Deshalb sind diese in der Regel vorzuziehen [166-168].

\section{Komplikationen bei der PCI}

\subsection{Dissektionen}

Während einer PCI kann es zu einer Koronardissektion kommen, die durch einen Einriss der Gefäßintima charakterisiert wird und eine Flusslimitation bis hin zu einem Gefäßverschluss zur Folge haben kann (s. Beispiel in - Abb. 25). Dissektionen können entweder durch den Führungskatheter selbst, durch den Führungsdraht, die Ballondilatation einer Koronarstenose bzw. durch die Implantation eines Stents verursacht werden [169-172]. Das Ausmaß 


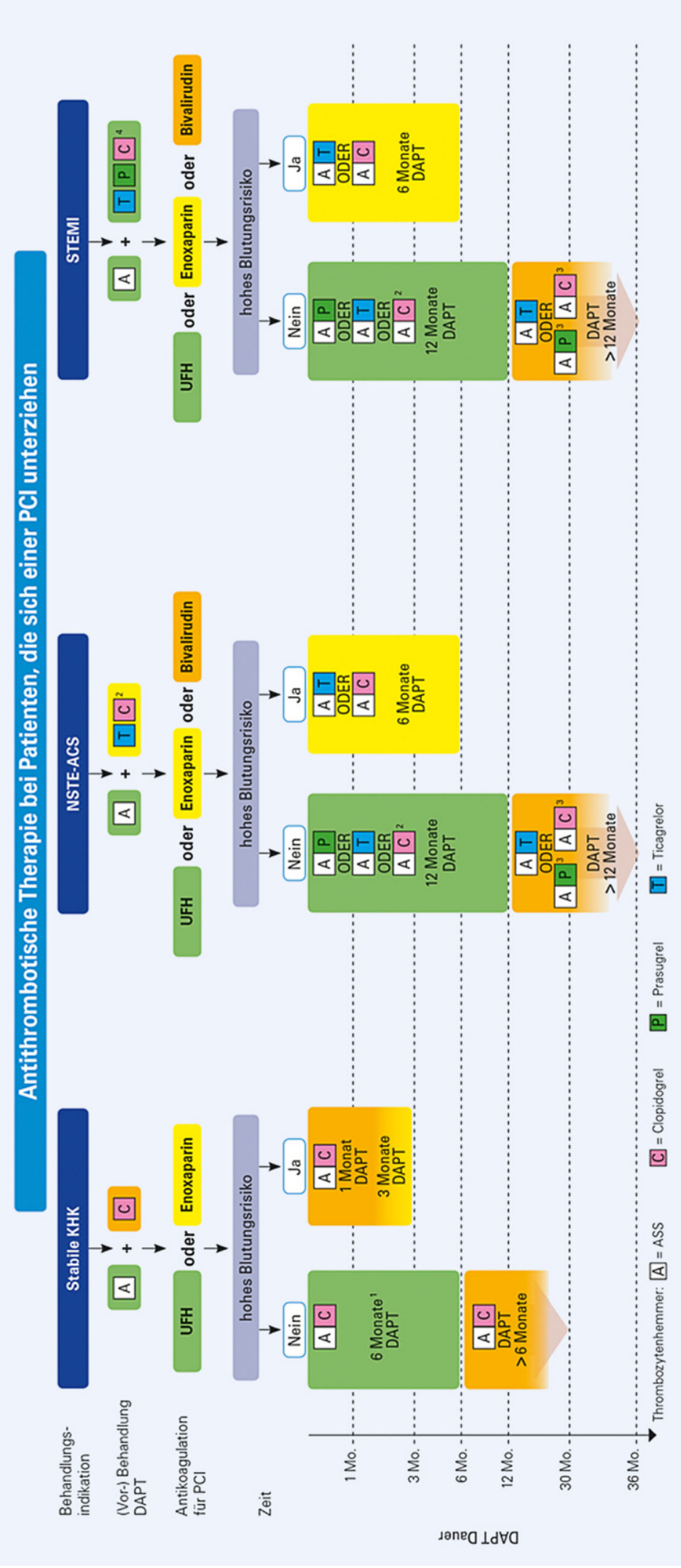

Abb. $23 \triangleleft$ Antithrombotische Therapie bei Patienten nach PCI [6, 45]. Mo. = Monat(e). Die Farbkodierung bezieht sich auf die ESC-Empfehlungsgrade (grün = Grad l; gelb = Grad lla; orange $=\mathrm{Grad} \mathrm{Ilb)}$. ${ }^{1} \mathrm{Nach}$ einer $\mathrm{PCI}$ mit DCB sollte eine 6-monatige DAPT erwogen werden (Ila). ${ }^{2}$ Clopidogrel, falls Patient nicht für eine Behandlung mit Prasugrel oder Ticagrelor infrage kommt, oder in einer Situation der DAPT-Deeskalation (llb). ${ }^{3}$ Clopidogrel oder Prasugrel, falls Patient nicht für eine Behandlung mit Ticagrelor infrage kommt. ${ }^{4}$ Vorbehandlung vor $\mathrm{PCl}$ (oder spätestens zum Zeitpunkt der PCl); Clopidogrel, falls potente $\mathrm{P} 2 \mathrm{Y}_{12}$-Hemmer kontraindiziert oder nicht verfügbar sind

der Koronardissektion korreliert mit dem Risiko für einen Gefäßverschluss und bestimmt entsprechend die Notwendigkeit einer therapeutischen Konsequenz. Das National Heart, Lung and Blood Institute teilt die Koronardissektion entsprechend ihrer Ausprägung in 6 Typen ein (s. Zusatzmaterial Online zusätzliche Tab. 17; $[171,172])$. Um das Risiko einer weiteren Ausbreitung der Dissektion und ggf. die Perfusion des "falschen“ Lumens zu reduzieren, sollte die Dissektion in den meisten Fällen durch einen Stent abgedeckt werden $[169,170]$. Wichtig ist es hierbei, eine korrekte Lage des Koronarführungsdrahtes im „wahren" Lumen der Koronararterie sicherzustellen, bevor ein Stent implantiert wird. Die Überprüfung der korrekten Lage des Koronardrahtes kann hierbei durch eine distale Kontrastmittelanfärbung über einen Mikrokatheter erfolgen [169, 170].

Nach Auftreten einer Koronardissektion infolge einer $\mathrm{PCl}$ sollte unter allen Umständen vermieden werden, den Koronardraht aus dem Zielgefäß zu ziehen, da bei einem erneuten Einbringen des Drahtes die Gefahr besteht, das falsche Lumen zu sondieren und das Gefäß hierdurch zu verschließen [169, 170].

Besteht nach einer PCl Unsicherheit über das Vorliegen einer Dissektion am Stenteingang oder Stentausgang, sollte eine intravaskuläre Bildgebung erfolgen, um das Vorhandensein bzw. das Ausmaß einer Dissektion zu beurteilen und ggf. eine therapeutische Konsequenz hieraus abzuleiten [170]. 


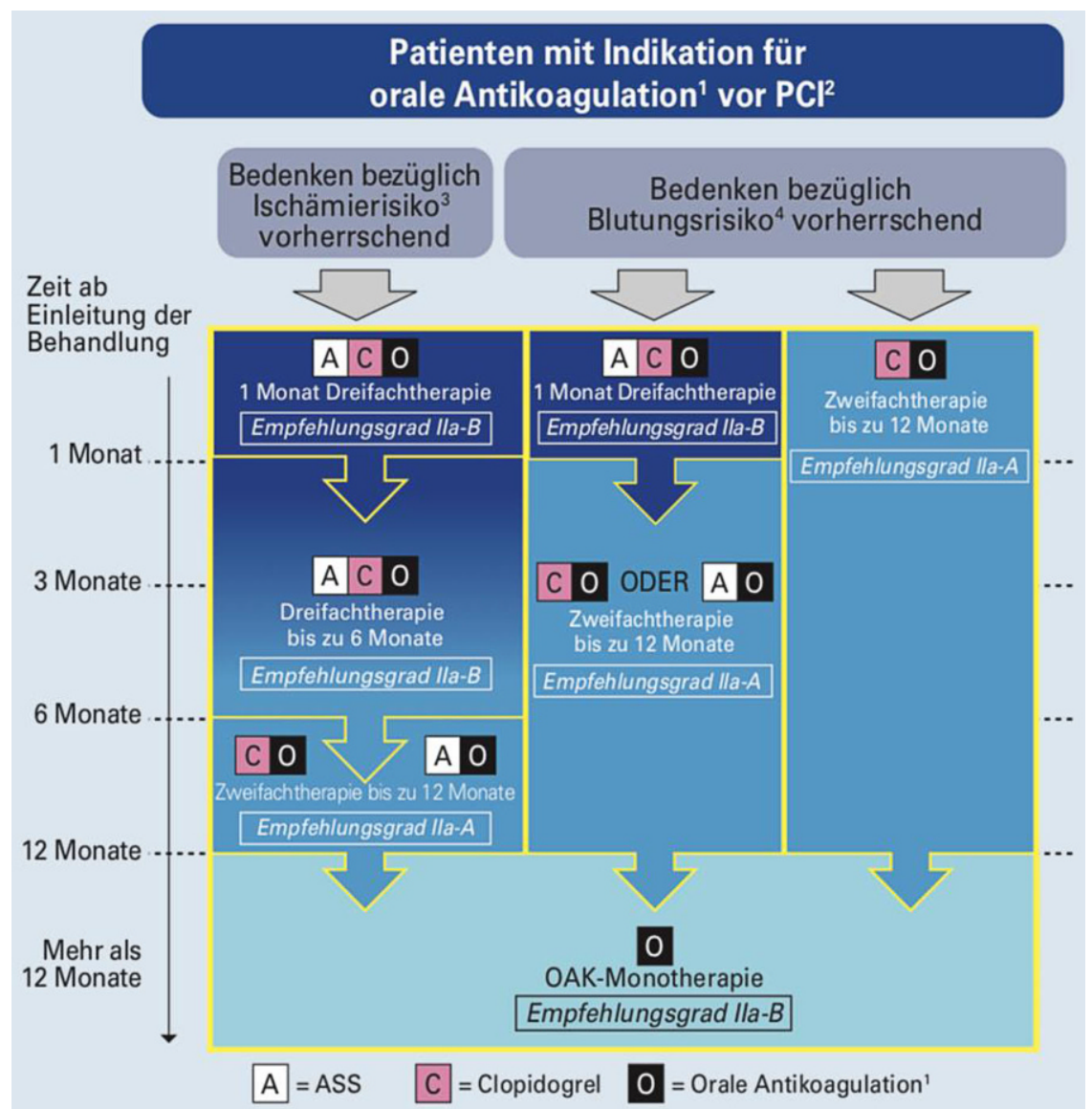

Abb. 24 \ Antithrombotische Therapie bei Patienten mit bestehender Antikoagulation. ${ }^{1}$ Nicht-Vitamin-K-basiertes orales Antikoagulans (NOAK) ist VKA bei Patienten mit nicht-valvulärem AF vorzuziehen. (Empfehlungsgrad Ila-A). ${ }^{2}$ Periprozedurale Gabe von ASS und Clopidogrel während der PCl wird unabhängig von der Behandlungsstrategie empfohlen. ${ }^{3}$ Als hohes Ischämierisiko wird angesehen akute klinische Präsentation oder anatomische/prozedurale Merkmale, die das Risiko für Myokardinfarkt erhöhen könnten. ${ }^{4}$ Das Blutungsrisiko kann mittels HAS-BLED- oder ABC-Score ermittelt werden. (Mod. nach [6])

\section{2 "No-reflow"-Phänomen}

Das „No-Reflow"-Phänomen beschreibt eine reduzierte oder ausbleibende Reperfusion einer Koronararterie infolge einer $\mathrm{PCl}$ und tritt in mehr als $20 \%$ der Fälle nach primärer $\mathrm{PCl}$ im akuten Koronarsyndrom und in bis zu $2 \%$ nach $\mathrm{PCl}$ im Rahmen einer stabilen KHK auf [173]. Ursachen für eine ausbleibende Reperfusion nach PCI können v.a. eine distale atherothrombotische Embolisation oder eine mikrovaskuläre Endothelschädigung infolge der Myokardischämie bzw. durch die Reperfusion selbst sein. Prädiktoren für den "No-Reflow" sind die Dauer der myokardialen Ischämie sowie eine verlängerte "Doorto-Ballon"-Zeit beim akuten Koronarsyndrom, $\mathrm{PCl}$ von Venengrafts wie auch eine hohe Thrombuslast im Zielgefäß bzw. lipidreiche Läsionen [173]. Für die Einleitung therapeutischer Maßnahmen ist es zunächst erforderlich zu unterscheiden, ob die Ursache des „No-Reflow “im Bereich der epikardialen Gefäße oder mikrovaskulär zu finden ist. Ursachen im Bereich der großen epikardialen Gefäße können eine Dissektion, ein Thrombus oder ein Plaqueshift sein [173-175]. Weiterhin können ein Koronarspasmus wie auch eine Luftembolie zu einer verzögerten oder ausbleibenden Reperfusion führen. Zunächst sollte die „activated clotting time“ (ACT) überprüft werden und eine Spülung des Zielgefäßes mit $\mathrm{NaCl}$-Lösung bzw. die intrakoronare Injektion von Nitro erfolgen (I, C). Weiterhin kann unter Berücksichtigung des Blutungsrisikos die Gabe von Glykoprotein-Ilb/Illa-Antagonisten evaluiert werden [173-175].
Liegt die Ursache des "No-Reflow" in einer thrombotischen Verlegung des Zielgefäßes, kann eine Thrombusaspiration in Betracht gezogen werden. Wird eine thrombotische Verlegung der myokardialen Mikrozirkulation angenommen, können Vasodilatatoren, wie z.B. Adenosin oder Verapamil, i.c. appliziert werden. Adenosin wirkt hierbei vasoaktiven Substanzen (u.a. Endothelin und Leukotriene) entgegen, wodurch der koronare Abfluss verbessert werden kann; ein positiver Einfluss auf die Infarktgröße oder Mortalität konnte in bisherigen Studien allerdings nicht eindeutig bestätigt werden [176].

\subsection{Blutungen}

Bei Blutungen, die während oder nach einer Koronarangiographie auftreten, handelt es sich häufig um Komplikationen der Zugangswege. Hierbei sollten oberflächliche Haut- oder subkutane Blutungen von potenziell lebensbedrohlichen Blutungskomplikationen unterschieden werden $[177,178]$. Zu den lebensgefährlichen Blutungen gehören v.a. retroperitoneale Blutungen, die mit einer Inzidenz von $0,1-0,7 \%$ beim femoralen Vorgehen beschrieben werden [177, 178]. Problematisch ist hierbei, dass retroperitoneale Hämatome lange klinisch inapparent bleiben oder mit unspezifischen Symptomen (Rückenschmerzen, Flankenschmerzen) einhergehen und sich häufig erst spät durch einen hämorrhagischen Schock klinisch manifestieren. Bei klinischem Verdacht auf eine retroperitoneale Blutung nach einem Kathetereingriff (femoraler Zugang) sollten daher eine rasche Bildgebung mittels CT und ein intensivmedizinisches Monitoring sowie die Bereitstellung von Erythrozytenkonzentraten erfolgen. Unter Berücksichtigung von Bildgebung und klinischer Symptomatik sollte rasch eine Entscheidung getroffen werden, ob interventionell oder gefäßchirurgisch versucht werden sollte, die Blutung zu stoppen [177-179].

\subsection{Perforation}

Die Koronarperforation infolge einer $\mathrm{PCI}$ (Ballondilatation oder Stentimplantation) ist eine schwerwiegende Komplikation, die mit einer Häufigkeit von bis zu 0,4\% 
Hier steht eine Anzeige.

黑 Springer 


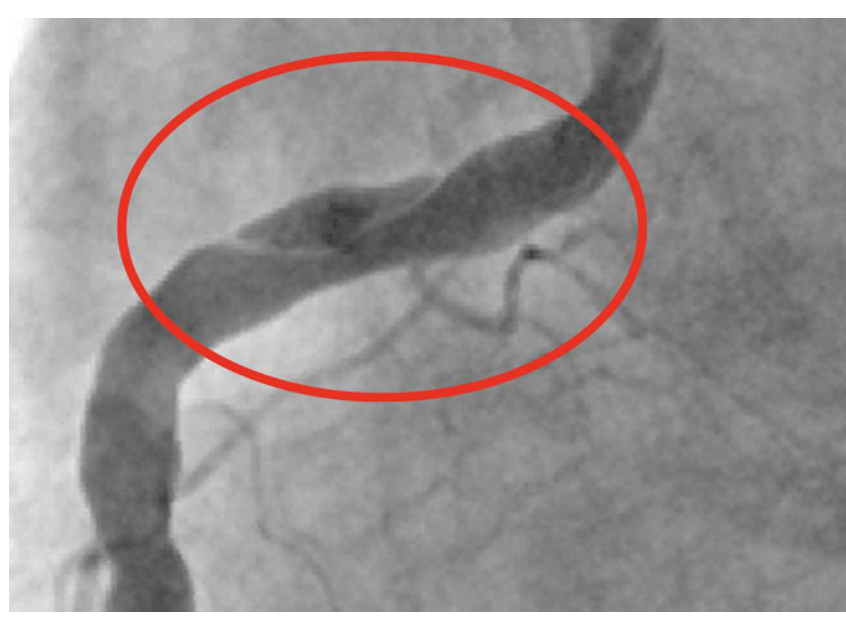

Abb. 25 ॥ Dissektion im Bereich des RCA-Ostiums (a.e. durch den Katheter)

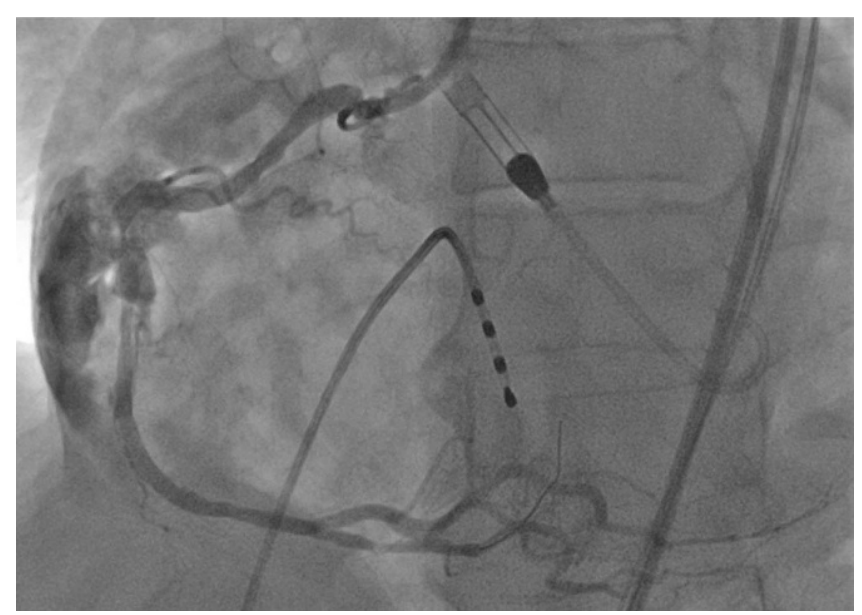

Abb. $26 \Delta$ Koronarperforation nach Rotablation im Bereich der RCA beschrieben wird (s. Beispiel in - Abb. 26; $[180,181])$. Risikofaktoren für eine Perforation sind ein Missverhältnis zwischen Ballon- bzw. Stentdiameter und Gefäßdiameter wie auch starke Gefäßverkalkungen. Weiterhin können Gefäßperforationen durch den Einsatz von sog. Cutting-/ Scoring-Ballons oder Hochdruckballons auftreten [180, 182, 183].

Weitere Risikofaktoren für eine Koronarperforation sind ein hohes Alter, das weibliche Geschlecht oder die PCl eines BypassGrafts [180, 182, 183]. Die Koronarperforation wird entsprechend ihrer Ausprägung nach der sog. Ellis-Klassifikation eingeteilt, wobei bei Auftreten einer Klasse-III-Perforation das Risiko einer Perikardtamponade hoch ist, die einer raschen Perikardpunktion bedarf (s. Zusatzmaterial Online zusätzliche Tab. 18; [184]).

Nach Auftreten einer Koronarperforation sollte die sofortige Abdeckung der Perforationsstelle durch einen Ballon erfolgen, um zu verhindern, dass Blut aus dem Koronarsystem austritt. Die Inflation des Ballons sollte für mehrere Minuten aufrechterhalten werden, da bei geringem Ausmaß der Perforation (Ellis I) bereits eine Stillung der Blutung aus dem Gefäß erreicht werden kann. Bei schwerwiegenderen Blutungen (Ellis II, III) sind häufig weitere Maßnahmen erforderlich, wie z.B. die Implantation eines "gecoverten" Stents. Hierbei muss in manchen Fällen die sog. „Ping-Pong“-Technik eingesetzt werden, um einen Blutverlust über die Perforation möglichst gering zu halten (s. Zusatzmaterial Online zusätzliche Tab. 19). Dies bedeutet die zusätzliche
Verwendung eines weiteren Führungskatheters, über den der "gecoverte"-Stent vorgebracht werden kann, ohne dass der Abdeckungsballon entfernt werden muss (s. - Abb. 27).

Bei der Perforation distaler Gefäßabschnitte muss eine Coil-Embolisation des Gefäßes in Betracht gezogen werden. Bei frustraner Implantation eines gecoverten Stens in eine proximale Lokalisation der Perforation muss eine chirurgische Therapieoption diskutiert werden [180-182, 184].

\subsection{Stentverlust}

Der Verlust eines Stents im Rahmen einer $\mathrm{PCl}$ ist eine seltene Komplikation und tritt mit einer Häufigkeit von $<0,3 \%$ auf $[185,186]$. Das Risiko hierbei besteht in der Embolisation des Devices in das Koronarsystem und das hiermit verbundene erhöhte Risiko einer Thrombose so wie auch das Risiko der Embolisation des Devices in den Kreislauf oder der zerebralen Strombahn. Ursachen für einen Stentverlust können stark atherosklerotisch veränderte oder auch stark torquierte Gefäße sein, wodurch der Stent beim Vorbringen in das Gefäß oder beim Rückzug (insbesondere in den Führungskatheter) vom Stentballon abgelöst werden kann [185-187]. Es gibt verschiedene interventionelle Techniken, um ein „verlorenes" Device aus einer Koronararterie zu bergen. Bei der Auswahl der interventionellen Technik ist die Lage des Koronardrahtes von Bedeutung. So ist zunächst zu prüfen, ob der Stent weiterhin auf dem Koronardraht liegt [185-187]. Ist dies der Fall, kann versucht werden, einen kleinkalibrigen Ballon (Diameter: 1-2 mm) über den Draht in den Stent vorzubringen und den Stent zu inflatieren. Danach kann eine Bergung des Stents mit dem Ballon über den Führungsdraht erfolgen [187]. Gelingt dies nicht, kann der embolisierte Stent über eine Schlinge (Snare) geborgen werden. Eine weitere interventionelle Technik zur Bergung eines verlorenen Stents ist die "Multiwire Technique"; hierbei werden mehrere Koronarführungsdrähte an dem embolisierten Stent vorbei in das Zielgefäß eingebracht und dann umeinander verdreht, sodass sich das Device hierin verfängt und aus dem Gefäß herausgezogen werden kann [185-187]. Scheitern die verschiedenen interventionellen Techniken, um das Device zu bergen, kann der embolisierte Stent durch Implantation eines weiteren Stents "gecrushed" und hierdurch an die Wand der Koronararterie gepresst werden. Aufgrund des hiermit einhergehenden erhöhten Risikos für Stentthrombosen sollte eine verlängerte Dauer der Thrombozytenaggregationshemmung erwogen werden [185].

\subsection{Aortale Dissektion}

Eine Koronardissektion im Bereich der proximalen Gefäßabschnitte kann sich sowohl nach antegrad als auch nach retrograd fortsetzen und bis in die Aorta ascendens reichen [188, 189]. Da eine Dissektion der Aorta ascendens generell als ein potenziell lebensbedrohliches Ereignis angesehen 


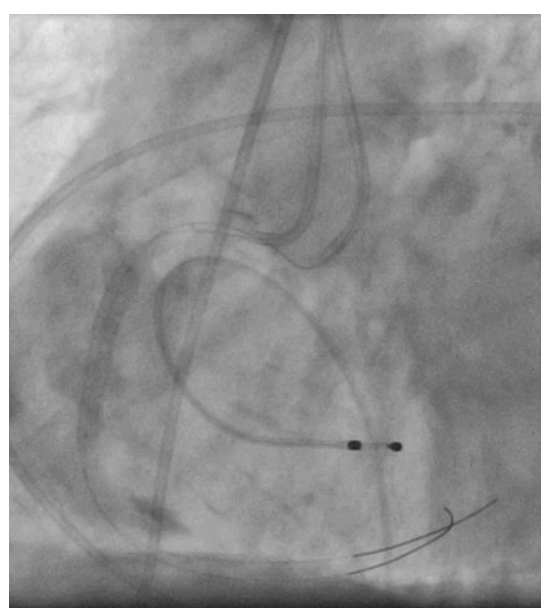

Abb. 27 A Ping-Pong-Manöver bei koronarer Perforation

werden muss, sollte hier zwischen einer Läsion, die einer unmittelbaren chirurgischen Therapie, einer interventionellen Behandlung oder einer möglichen konservativen Therapie bedarf, unterschieden werden. Zur Beurteilung der Ausprägung und der Abschätzung der notwendigen therapeutischen Konsequenz kann die DunningKlassifikation hilfreich sein (s. Zusatzmaterial Online zusätzliche Tab. 16 sowie Beispiel in D Abb. 28; [188-190]). Breitet sich die Koronardissektion retrograd in die Aorta ascendens weniger als $4 \mathrm{~cm}$ aus (Dunning I), ist eine PCl mit Abdeckung des Entry im Bereich des aortoostialen Übergangs häufig ausreichend. Bei einer Fortsetzung der Dissektion bis in die Aorta ascendens mit einer Ausbreitung von $\geq 4 \mathrm{~cm}$ (Dunning II) sollte eine chirurgische Behandlung diskutiert werden. Bei unklarem Befund bzw. zweifelhafter Einschätzung der Ausdehnung der Aortendissektion sollte eine erweiterte Bildgebung mittels TEE oder CT erfolgen [188-190].

\subsection{Neurologische Komplikationen}

Eine zerebrale Embolie oder eine intrazerebrale Blutung im Rahmen einer Katheteruntersuchung ist als schwerwiegende Komplikationen zu betrachten. Klinische Symptome können hierbei das Auftreten von Paresen (35\%), Sprachstörungen (33\%), psychomotorische Störungen (32\%), Sehstörungen (15\%) und einer Mundastschwäche (15\%) sein [191-193]. Nach Auftreten der entsprechenden Symptomatik sind eine zeitnahe neurologi-

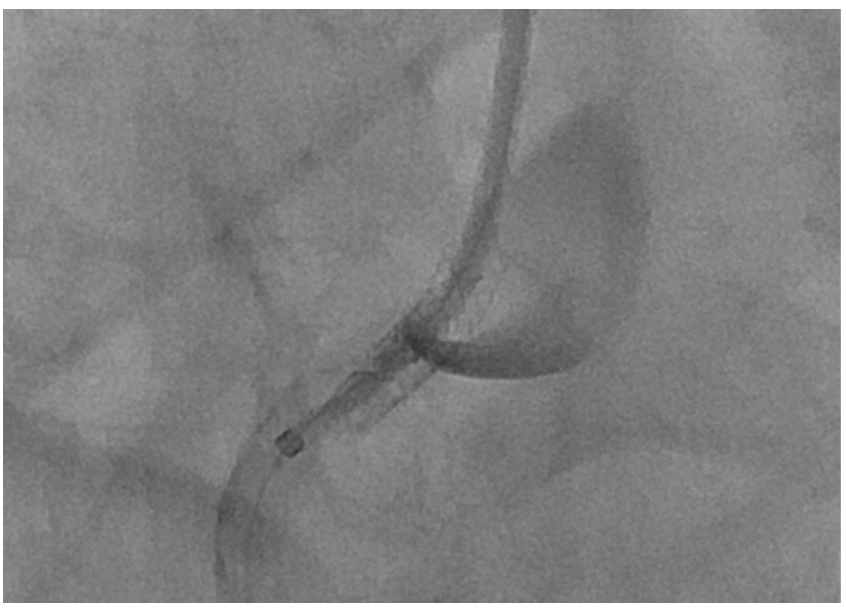

Abb. $28<$ latrogene Aortendissektion Dunning I sche Beurteilung mit Bestimmung des Schweregrads des neurologischen Defizits sowie die Unterscheidung zwischen einem ischämischen Schlaganfall und einer intrazerebralen Blutung oder auch anderer neurologischer Komplikation (z. B. Luftembolie, kontrastmittelinduzierte fokale Funktionsstörung) notwendig [191]. Daher sollte unmittelbar ein Neurologe hinzugezogen und eine zerebrale Bildgebung durchgeführt werden $(I, C)$. Unter Berücksichtigung des nachgewiesenen Befundes, der örtlichen Gegebenheiten und der Zeitdauer seit dem Auftreten der neurologischen Symptomatik sowie des Ausmaßes des neurologischen Defizits sollte eine spezifische Therapie (Antikoagulation, Lyse, Thrombektomie, Operation o. Ä.) in Abstimmung mit dem Neurologen eingeleitet werden $(\mathrm{I}, \mathrm{C})$ [191-193].

\section{Heart-Team}

\subsection{Informationsaustausch - Heart- Team-Konferenz}

Für eine gute Indikationsstellung zur kardiologischen oder kardiochirurgischen Intervention sind auch für den Kardiologen Kenntnisse der kardiochirurgischen Techniken und Therapiemöglichkeiten erforderlich.

Hierfür ist ein regelmäßiger Erfahrungsaustausch notwendig, der durch formale Kontakte mit einer herzchirurgischen Klinik am Hause oder in erreichbarer Nähe (s. unten) geregelt sein sollte $(\mathrm{l}, \mathrm{C})$.
Empfehlenswert ist eine kardiologischkardiochirurgische Konferenz zumindest über diejenigen Patienten, für die sowohl eine $\mathrm{PCl}$ als auch eine ACB-Operation als Therapiemaßnahme infrage kommt. Dementsprechend sollte von einer $\mathrm{Ad}-\mathrm{hoc}-\mathrm{PCl}$ bei diesen Patienten (z. B. Hauptstammintervention, Mehrgefäßerkrankung) abgesehen werden $(I, C)$. Andernfalls kann auch ein im Vorfeld festgelegtes Standardprozedere als Entscheidungshilfe dienen. Muss aus bestimmten Gründen eine unmittelbare $\mathrm{PCl}$ im Anschluss an eine Koronarangiographie erfolgen, erfolgt im besten Fall eine kurze gemeinsame Falldiskussion mit den Herzchirurgen im Herzkatheterlabor.

Eine Heart-Team-Besprechung sollte auch für Problempatienten z. B. bei schwieriger oder risikoreicher $\mathrm{PCl}$ und/oder $\mathrm{ACB}$ genutzt werden. Neben der Fallbesprechung kann sie auch zur Kommunikation der neuesten Techniken und Indikationsstellungen sowie zur Diskussion von Komplikationen bei der $\mathrm{PCl}$ oder ACBOperation im Rahmen einer "Morbiditätund Mortalitätskonferenz" genutzt werden. Diesbezüglich sind allerdings Regeln zur Durchführung im Vorfeld zu definieren [6]. Ein wissenschaftlicher Austausch kann wöchentlich auf der Basis eines JournalClubs erfolgen.

\subsection{Strukturierte Notfallbereitschaft}

Grundsätzlich ist eine strukturierte Kooperation mit einem kardiochirurgischen Zentrum erforderlich, damit im Notfall eine 
Operation schnellstmöglich erfolgen kann $(\mathrm{I}, \mathrm{C})$. Die Planung einer Operationsbereitschaft sollte alle Beteiligten (Kardiologen, Herzchirurgen, Anästhesisten, Transportwesen) einbeziehen. Die Vorhaltung eines entsprechenden Ablaufprotokolls im HKL ist zu empfehlen (I, C).

Elektive Hochrisiko-PCls (Hauptstammintervention, „last remaining vessel“, komplex chronisch totale Läsion) sollten nur in erfahrenen Zentren durchgeführt werden. Bei hochkomplexen Eingriffen sollte die Möglichkeit zur Durchführung einer mechanischen Kreislaufunterstützung vorhanden sein $(I, C)$.

\section{Strukturierte Begleit- maßnahmen}

\subsection{Aufklärung und Einverständnis- erklärung}

Der Patient muss grundsätzlich in einem Gespräch von einem qualifizierten Arzt über die Art, den Umfang sowie die Durchführung und den Nutzen (also die Notwendigkeit, Dringlichkeit und Eignung), die dem Eingriff spezifisch anhaftenden Risiken - einschließlich der Risikohäufigkeit - und Folgen der geplanten diagnostischen Herzkatheteruntersuchung sowie einer möglichen Intervention als auch über Alternativen und andere Therapieoptionen und deren Möglichkeiten und Risiken informiert werden. Sprachlich und inhaltlich sind dabei die konkrete Situation des Patienten und sein allgemeines Krankheitsverständnis zu berücksichtigen. Die Aufklärung muss so rechtzeitig erfolgen, dass der Patient seine Entscheidung über die Einwilligung wohlüberlegt treffen kann. Daher sollte das Aufklärungsgespräch spätestens am Tag vor der Untersuchung durchgeführt werden. Eine Aufklärung unmittelbar vor Beginn der Herzkatheteruntersuchung ist grundsätzlich unzulässig. Bei Notfalleingriffen oder vitaler Indikation, die eine sofortige Behandlung des Patienten erfordern, kann die Aufklärung entbehrlich sein und der Eingriff über die Grundsätze der mutmaßlichen Einwilligung gerechtfertigt werden.

Bei der Durchführung des Aufklärungsgesprächs sollte ein standardisierter juristisch geprüfter Aufklärungsbogen verwen- det werden, der durch handschriftliche Einträge individualisiert werden sollte.

Abschließend bestätigt der Patient durch seine Unterschrift, dass er ausführlich informiert wurde, persönliche Fragen geklärt werden konnten, mögliche Komplikationen besprochen wurden, er den Inhalt des Aufklärungsgespräches und des Aufklärungsbogens verstanden hat und somit in die geplante Untersuchung einwilligt. Der Patient sollte darauf hingewiesen werden, dass er die Einwilligung bis zur Untersuchung jederzeit widerrufen kann.

Datum und Uhrzeit der Aufklärung sind zu notieren, und dem Patienten ist eine Kopie des Aufklärungsbogens auszuhändigen. Sollten handschriftliche Anmerkungen des Arztes nachträglich erfolgen, so sind diese auch auf der Kopie zu vermerken und von Arzt und Patient gegenzuzeichnen. Das Original verbleibt in der Patientenakte.

Auch unter Geltung des Patientenrechtegesetzes (vgl. § 630 e II 1 Nr. 1 BGB) kann die Aufklärung des Patienten durch einen Nichtfacharzt erfolgen. Der aufklärende Arzt muss jedoch ausreichend mit Nutzen und Risiken der geplanten Prozedur vertraut sein und die für die Aufklärung erforderliche Qualifikation besitzen [194-196]. In jedem Fall muss der untersuchende Arzt sich vor Untersuchungsbeginn vergewissern, dass der Patient ordnungsgemäß aufgeklärt wurde (I, C).

\subsection{Monitorüberwachung nach Intervention}

Eine grundsätzliche Monitorüberwachung nach elektiver $\mathrm{PCl}$ muss nicht erfolgen. Allerdings kann im Einzelfall nach komplexer Prozedur (Hauptstamm, CTO, Rotablation) eine Überwachung für $24 \mathrm{~h}$ erwogen werden. Bei Patienten mit NSTE-ACS und PCl ist eine Überwachung für $24 \mathrm{~h}$ bei niedrigem Risiko für das Auftreten von Rhythmusstörungen zu erwägen (I, C). Bei hohem Risiko sollte eine Überwachung $>24 \mathrm{~h}$ auf einer Intensivstation oder IMC erfolgen (I, C). Bei Patienten mit STEMI sollte in jedem Fall eine Überwachung für mindestens $24 \mathrm{~h}$ nach Symptombeginn durchgeführt werden $(I, C)[45,46,104]$.

\subsection{Schleusenentfernung nach Koronarangiographie}

Nach einer Koronarangiographie sollte die Schleuse durch den Arzt oder geschultes Personal rasch entfernt werden. Koronarangiographien über die $\mathrm{A}$. radialis werden in der Regel mit subtherapeutischer Heparinisierung (2500-5000 IE) durchgeführt. Durch die gute Kompressionsmöglichkeit $\operatorname{der}$ A. radialis kann die Schleuse sofort entfernt werden. Hierzu eignen sich spezielle zirkuläre Kompressionsbänder, die die Durchblutung der Hand gewährleisten. Nach Anlegen des Kompressionsbandes muss die Punktionsstelle verschlossen sein und die periphere Durchblutung durch Offenhalten der A. ulnaris gewährleistet sein. Nach 2-4 h kann die Kompression entfernt und der Puls kontrolliert werden. Ein lockerer Druckverband sollte angelegt und die Hand für 3 bis 5 Tage geschont werden. Eine ärztliche Pulskontrolle am nächsten Tag ist obligat und sollte dokumentiert werden.

Die Koronarangiographie via A. femoralis wird in der Regel ohne systemische Heparinisierung durchgeführt, sodass die Schleuse unmittelbar entfernt werden und das Gefäß manuell komprimiert werden kann. Alternativ bieten sich Verschlusssysteme an. Alternativ können passive Kompressionssysteme verwendet werden. Eine Bettruhe über $3 \mathrm{~h}$ mit standardisiertem Druckverband ist zu gewährleisten.

\subsection{Schleusenentfernung nach PCI}

Die therapeutische Heparinisierung, größere Schleusen bzw. mehrere Schleusen (CTO) und Thrombozytenaggregationshemmung erhöhen die Gefahr intraund postprozeduraler Blutungen, welche mit einer erhöhten Mortalität verbunden sind. Eine engmaschige Überwachung des Patienten gewährleistet ein schnelles Erkennen und Handeln.

Nach $\mathrm{PCl}$ über die A. radialis sollte, um Gefäßverschlüsse zu vermeiden, trotz therapeutischer Heparinisierung die Schleuse unverzüglich entfernt werden. Es kann genauso wie nach diagnostischer Koronarangiographie verfahren werden, wobei die Kompression verlängert werden kann. Eine Bettruhe ist nicht zwingend erforderlich. 
Hier steht eine Anzeige.

黑 Springer 
Nach PCl über die A. femoralis sind aktive Verschlusssysteme zu bevorzugen, da sie eine sofortige Blutstillung trotz Heparinisierung ermöglichen. Alternativ ist ein Schleusenzug nach Abklingen des Heparins mit manueller Kompression oder passivem Verschlusssystem möglich. Nach erfolgreicher Blutstillung sollte ein standardisierter Druckverband angelegt werden. Der Patient muss eine Bettruhe für 3-6h einhalten. Bei größeren Schleusen (7 und 8F), Gefäßkomplikationen oder fortgesetzter oraler Antikoagulation können längere Liegezeiten (6-12h) erforderlich sein. Nach Entfernen des Druckverbands sollte die Leiste in Augenschein genommen und auskultiert werden, um Aneurysmata spuria und Gefäßfisteln auszuschließen (l, C). Bei stärkeren Nachblutungen und/ oder einem Hämoglobinabfall von mehr als $3 \mathrm{~g} / \mathrm{dl}$ sollte ein Gefäßchirurg konsultiert werden $(\mathrm{I}, \mathrm{C})$, ggf. sind eine Transfusion und/oder eine operative oder interventionelle Gefäßrevision vorzunehmen. Eine retroperitoneale Blutung kann ohne lokale Beschwerden auftreten und kann mit einem hämorrhagischen Schock einhergehen und muss ggf. als Notfall chirurgisch oder interventionell behandelt werden. Ein Verdacht erfordert zur Klärung eine sofortige Bildgebung.

\subsection{Ambulante und stationäre Nachsorge}

Nach PCl werden die Patienten in der Regel in einer zentralen Überwachungseinheit bis zur Verlegung auf Station überwacht. Diese Einheit sollte möglichst nah am Herzkatheterraum liegen, um bei Komplikationen die personellen und apparativen Ressourcen nutzen zu können. Die Nachsorge sollte von qualifiziertem Personal, welches mit der Überwachung von Herzkatheterpatienten vertraut ist, durchgeführt werden. Das Personal sollte besonders kardiologisch geschult im Umgang mit den passiven radialen und femoralen Kompressionssystemen und der Anlage und Kontrolle von Druckverbänden sein. Komplikationen wie Nachblutungen mit hämorrhagischem Schock, neurologische Komplikationen und allergische Reaktionen sollten rasch erkannt werden. Hier kann ggf. die Einrichtung eines sog. „Rap-
id-Response"-Teams zur Optimierung der strukturellen Abläufe dienen.

Bei auffälligen Beschwerden oder Befunden sollte die sofortige Konsultation des interventionellen Kardiologen erfolgen (I, C). Der Nachsorgebereich sollte über eine Mindestausstattung mit EKGund Blutdruckmonitoring, Oxymetrie, Sauerstoffgabe und eine zentrale Rufanlage verfügen. Eine komplette Notfallausrüstung mit Absaugeinrichtung und Defibrillator müssen verfügbar sein.

Am Ende der Nachbeobachtung bei PCl-Patienten sollte ein EKG mit $12 \mathrm{Ab}$ leitungen registriert werden (Ila, C). Die $\mathrm{PCl}$ über die A. radialis oder die A. femoralis erfordern eine Nachbetreuung in der Regel bis zum nächsten Tag oder bis $24 \mathrm{~h}$. Der Kreatininwert bzw. die GFR sollte bei Patienten mit eingeschränkter Nierenfunktion oder bei hoher Kontrastmittelmenge vor Entlassung kontrolliert werden. Im Abschlussgespräch sollte nochmals der Stentpass kontrolliert und auf die Wichtigkeit der exakten Einnahme der DAPT hingewiesen werden.

\subsection{Dokumentation}

Die Herzkatheteruntersuchung und $\mathrm{PCI}$ erfordern eine lückenlose Dokumentation der Untersuchung. Der untersuchende Arzt, das Assistenzpersonal, die genaue Uhrzeit und die Dauer der Untersuchung müssen auf dem Protokoll dokumentiert werden. Die Untersuchung sollte ähnlich einer Operation, beginnend mit Punktion und endend mit dem Gefäßverschluss, chronologisch protokolliert werden. Alle verwendeten Materialien sind zu dokumentieren. Der ausgewählte Stenttyp, Länge und Durchmesser sowie Implantationsdruck mit dem erreichten Stentlumen sollten gut ersichtlich sein. Die Dokumentation der Strahlendosis und der Strahlendauer ist gesetzlich vorgeschrieben. Der Angabe des genauen Kontrastmittelverbrauchs ist für die Qualitätssicherung obligat. Schwierige Untersuchungsbedingungen sind zu beschreiben, um eine erhöhte Strahlenbelastung und Kontrastmittelmenge zu rechtfertigen. Insbesondere eine Strahlenbelastung über den gesetzlichen Referenzwert hinaus muss immer begründet werden (l, C).
Komplikationen und deren Behandlung sollten zeitlich in ihrem Auftreten protokolliert und beschrieben werden, um im Begutachtungsfall das Handeln des Arztes exakt nachvollziehen zu können. Das Protokoll sollte auch die erhobenen hämodynamischen Befunde umfassen. Eine ausreichende bildliche Dokumentation ist ebenfalls hilfreich. Die Implantation von passageren Devices (z. B. Impella etc.) sollte ebenfalls einmalig bildlich dokumentiert werden. Die Protokolle und Bilddateien müssen mindestens 10 Jahre archiviert werden. Zusätzlich ist der Untersucher nach Medizinprodukte-Betreiberverordnung (§ 15, Absatz 1, Nr. 2) sowie nach Verordnung (EU) 2017/745 über Medizinprodukte (Artikel 18) verpflichtet, nach der Implantation eines Stents oder der Behandlung mittels DEB dieses in einem Stentpass zu dokumentieren, der dem Patienten mitgegeben wird. Solche Pässe können von den Abteilungen selber gedruckt werden oder z. B. über die Deutsche Herzstiftung kostenlos bezogen werden.

Die heutige Software ist in der Regel mit dem Abteilungs- oder Klinikinformationssystem (KIS) und dem Herzkatheterplatz verbunden und protokolliert automatisch alle patientenbezogenen und prozeduralen Daten. Häufig werden Standardtexte verwendet, die editiert werden müssen. Die nötigen Qualitätsdaten sind in der Regel im Protokoll enthalten, sodass die Qualitätssicherung automatisch generiert wird. Bilddokumente sollten ggf. in den Befund eingebunden werden, sie sind hilfreich für weiterbetreuende Ärzte. Der Untersucher muss aber in jedem Fall auch die generierten Daten überprüfen, da er durch das Abzeichnen des Befundes für dessen Richtigkeit verantwortlich ist. Eine detaillierte Darstellung finden sich in den „Leitlinien zum Einrichten und Betreiben von Herzkatheterlaboren und Hybridoperationssälen/Hybridlaboren" [7].

\section{Assistenzpersonal}

\subsection{Allgemeines}

Das Assistenz- und Pflegepersonal sind ein essenzieller Bestandteil des KatheterTeams und entsprechend in das Team zu integrieren. Hierbei stehen die stetige Fortund Weiterbildung gemeinsam mit den 
Ärzten des Herzkatheterlabors im Mittelpunkt.

Hinsichtlich der Personalbedarfsermittlung des Assistenz- und Pflegepersonals im Herzkatheterlabor sind Art und Anzahl der Untersuchungen zu berücksichtigen. Neben einer Assistenz zur Dokumentation der Untersuchung und zur Bedienung der Registriereinheit im Routinebetrieb sollte eine zweite Assistenz als "Springer" im Herzkatheterlabor anwesend sein $(I, C)$, welche das Anreichen des Materials unterstützt. Im Rahmen einer PCl kann neben dem Untersucher eine sterile Assistenz zusätzlich am Tisch erwogen werden (Ilb, C).

\subsection{Aufgaben}

Das Assistenz- und Pflegepersonal ist mit einer Vielzahl administrativer, technischer und pflegerischer Aufgaben betraut. In der Regel ist es nicht nur bei der diagnostischen Koronarangiographie und der perkutanen Koronarintervention (PCI) beteiligt, sondern auch bei einer Vielzahl anderer Prozeduren (z. B. elektrophysiologische Untersuchungen und Interventionen, Schrittmacherimplantationen, diagnostische und interventionelle Prozeduren bei strukturellen Herzerkrankungen).

Eine weitere wichtige Tätigkeit des Assistenz- und Pflegepersonals ist die Rufbereitschaft sowie der notfallmäßige Einsatz beim akuten Koronarsyndrom.

\subsection{Auswahl- und Anforderungs- kriterien}

Bei der Personalauswahl ist die besondere Vielfalt der medizinischen, technischen und organisatorischen Aufgaben zu berücksichtigen, die vom Assistenz- und Pflegepersonal im Herzkatheterlabor bewältigt werden müssen. Die potenziellen Mitarbeiter sollten belastbar und ausgesprochen teamfähig sowie kommunikativ und patientenorientiert veranlagt sein. Des Weiteren sollten sie sich durch ihre grundsätzliche Bereitschaft zu Innovationen und Veränderungen auszeichnen.

\subsection{Aus- und Fortbildung}

Eine adäquate Ausbildung und kontinuierliche Fort- und Weiterbildung sind für die Qualifikation der Mitarbeiter im Herz- katheterlabor von entscheidender Bedeutung. Wichtig ist eine ausreichende Erfahrung in der Notfallmedizin, wie sie z.B. auf einer Intensivstation erworben wird. Durch die Kenntnis von Untersuchungsbefunden, Begleiterkrankungen und medikamentöser Therapie lassen sich die Risiken einer Herzkatheteruntersuchung minimieren. Das rechtzeitige Erkennen von Komplikationen und die angemessene Reaktion durch gut geschultes Personal können schwerwiegende Komplikationen abwenden. Die Abläufe während einer Notfallsituation sind regelmäßig zu trainieren, damit alle Maßnahmen im Ernstfall ohne Reibungsverluste geordnet vorgenommen werden können. Dazu zählen der fachkundige Umgang mit Notfallmedikamenten, die sachgerechte, sofortige Defibrillation bzw. das Legen einer temporären Schrittmachersonde und die fachgerechte Durchführung einer CPR inklusive manueller Thoraxkompression. Auch die speziellen Anforderungen bei Schockpatienten müssen bekannt sein, inklusive Indikationsstellung und Anwendung von Unterstützungssystemen (z. B. IABP, Impella oder ECMO).

Zusatzausbildungen betreffen Spezialaufgaben, die in einzelnen Katheterlabors anfallen (z.B. spezielle elektrophysiologische Kenntnisse, Schrittmachererfahrungen, Prozeduren bei strukturellen Herzerkrankungen u.a.). In diesem Zusammenhang ist eine Qualifizierung zum kardiologischen Fachassistenten wünschenswert.

\section{Qualitätssicherung}

Herzkatheteruntersuchungen und $\mathrm{PCl}$ werden heute im ambulanten und stationären Bereich sowohl durch Krankenhäuser als auch selbstständige Vertragsärzte erbracht. Häufig werden Patienten in beiden Sektoren versorgt. Der Gesetzgeber hat deshalb den gemeinsamen Bundesausschuss (G-BA) verpflichtet, eine sektorenübergreifende Qualitätssicherung zu entwickeln, um alle Herzkatheteruntersuchungen und $\mathrm{PCl}$ zu erfassen. Weiterhin sollen die Krankenkassen Sozialdaten liefern, um auch die Langzeitverläufe zu beurteilen. Auf die Erhebung der sektorenübergreifenden Qualitätssicherung mit Verwendung der individuellen Daten müssen die Patienten durch eine Pati- enteninformation hingewiesen werden. Die Datenerhebung erfolgt allerdings auf Grundlage der Vorgaben des 5. Buches des Sozialgesetzbuchs (SGB V), sodass keine Einwilligung der Patienten erforderlich ist.

Bei der Qualitätssicherung werden nicht nur prozedurale Daten erfasst, sondern auch klinische und diagnostische Daten, die auf die fachgerechte Indikation zur Herzkatheteruntersuchung abzielen.

Die Daten werden auf Landesebene meistens durch eigene unabhängige Einrichtungen ausgewertet und einer Fachkommission, bestehend aus invasiven Kardiologen, vorgelegt. Die vergleichende Auswertung wird den Krankenhäusern und Vertragsärzten zeitgerecht mitgeteilt. Bei signifikanten Abweichungen vom Durchschnitt werden die Krankenhäuser bzw. Vertragsärzte zu einer Stellungnahme aufgefordert, die dann von der Fachkommission beurteilt wird. Bei dauerhaften Auffälligkeiten können Sanktionen ausgesprochen werden. Einschränkend sollte erwähnt werden, dass diese Form der Qualitätssicherungspflicht bisher nur für gesetzlich versicherte Patienten gilt.

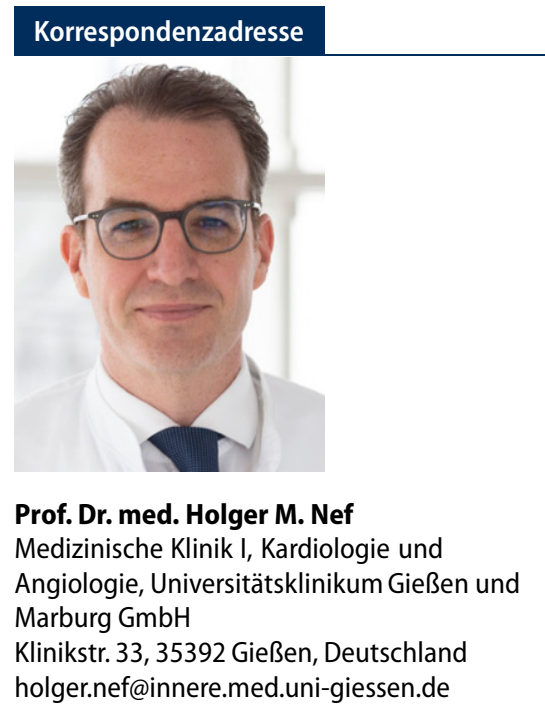

Danksagung. An dieser Stelle sei allen, die mit Unterstützung dazu beigetragen haben, dieses Manual zu finalisieren, herzlichst gedankt. Besonderer Dank gilt Frau Ulrike Esser, Abteilung Leitlinien.

\section{Einhaltung ethischer Richtlinien}

Interessenkonflikt. Den Interessenkonflikt der Autoren finden Sie online auf der DGK-Homepage unter 
http://leitlinien.dgk.org/ bei der entsprechenden Publikation.

Für diesen Beitrag wurden von den Autoren keine Studien an Menschen oder Tieren durchgeführt. Für die aufgeführten Studien gelten die jeweils dort angegebenen ethischen Richtlinien.

\section{Literatur}

\section{Verwendete Literatur}

1. Deutsche Herzstiftung (2018) Deutscher Herzbericht Bd. 30

2. Nef HM, Achenbach S, Birkemeyer $R$ et al (2021) Manual der Arbeitsgruppe Interventionelle Kardiologie (AGIK) der Deutschen Gesellschaft für Kardiologie - Herz- und Kreislaufforschung e. V. (DGK). Kardiologe. https://doi.org/10.1007/ s12181-021-00493-6

3. Bonzel T et al (2008) Percutaneous coronary interventions (PCI). Clin Res Cardiol 97(8):513-547

4. Hamm CW et al (2008) Diagnostic heart catheterization. Clin Res Cardiol 97(8):475-512

5. Patil D et al (2017) Appropriateness of elective percutaneous coronary intervention and impact of government health insurance scheme-A tertiary centre experience from Western India. Indian Heart J 69(5):600-606

6. Neumann FJ et al (2019) 2018 ESC/EACTS Guidelines on myocardial revascularization. Eur Heart J40(2):87-165

7. Schachinger V et al (2015) Guidelines to establish and operate catheterization laboratories and hybrid operating rooms/hybrid laboratories (3rd edition 2015). Kardiologe 9(1):89-123

8. Bonzel T, Wollschlager $H$, Just $H$ (1986) A new catheter system for the mechanical dilatation of coronary stenoses with exchangeable intracoronary catheters, fast flow of the contrast agent and improved control. Biomed Tech 31(9):195-200

9. Brophy JM, Belisle P, Joseph L (2003) Evidence for use of coronary stents. A hierarchical bayesian meta-analysis. Ann Intern Med 138(10):777-786

10. Carrie D et al (2001) Comparison of direct coronary stenting with and without balloon predilatation in patients with stable angina pectoris. Am J Cardiol 87(6):693-698

11. Ijsselmuiden AJ et al (2003) Direct coronary stenting compared with stenting after predilatation is feasible, safe, and more cost-effective in selected patients: evidence to date indicating similar late outcomes. Int J Cardiovasc Intervent 5(3):143-150

12. ljsselmuiden AJJ et al (2003) Direct coronary stent implantation does not reduce the incidence of instent restenosis or major adverse cardiac events: six month results of a randomized trial. Eur Heart J 24(5):421-429

13. Bondesson P et al (2012) Comparison of two drugeluting balloons: a report from the SCAAR registry. Eurolntervention 8(4):444-449

14. Scheller B et al (2006) Treatment of coronary instent restenosis with a paclitaxel-coated balloon catheter. NEngl J Med 355(20):2113-2124

15. Habara $S$ et al (2011) Effectiveness of paclitaxeleluting balloon catheter in patients with sirolimuseluting stent restenosis. JACC Cardiovasc Interv 4(2):149-154

16. Rittger $\mathrm{H}$ et al (2012) A randomized, multicenter, single-blinded trial comparing paclitaxel-coated balloon angioplasty with plain balloon angioplasty in drug-eluting stent restenosis: the PEPCAD-DES study. J Am Coll Cardiol 59(15):1377-1382

17. Habara $S$ et al (2013) A multicenter randomized comparison of paclitaxel-coated balloon catheter with conventional balloon angioplasty in patients with bare-metal stent restenosis and drug-eluting stent restenosis. Am Heart J 166(3):527-533

18. Byrne RA et al (2013) Paclitaxel-eluting balloons, paclitaxel-eluting stents, and balloon angioplasty in patients with restenosis after implantation of a drug-eluting stent (ISAR-DESIRE 3): a randomised, open-label trial. Lancet 381(9865):461-467

19. Alfonso $\mathrm{F}$ et al (2015) A prospective randomized trial of drug-eluting balloons versus everolimuseluting stents in patients with in-stent restenosis of drug-eluting stents: the RIBS IV randomized clinical trial. J Am Coll Cardiol 66(1):23-33

20. Cortese Betal (2010) Paclitaxel-coated balloon versus drug-eluting stent during $\mathrm{PCl}$ of small coronary vessels, a prospective randomised clinical trial. The PICCOLETO study. Heart 96(16):1291-1296

21. Latib A et al (2012) A randomized multicenter study comparing a paclitaxel drug-eluting balloon with a paclitaxel-eluting stent in small coronary vessels: the BELLO (Balloon Elution and Late Loss Optimization) study. J Am Coll Cardiol 60(24):2473-2480

22. Stella PR et al (2012) A multicenter randomized comparison of drug-eluting balloon plus baremetal stent versus bare-metal stent versus drug-eluting stent in bifurcation lesions treated with a single-stenting technique: six-month angiographic and 12-month clinical results of the drug-eluting balloon in bifurcations trial. Cathete Cardiovasc Interv 80(7):1138-1146

23. Lee MS et al (2002) Cutting balloon angioplasty. JInvasive Cardiol 14(9):552-556

24. Abdel-Wahab Met al (2013) High-speed rotational atherectomy before paclitaxel-eluting stent implantation in complex calcified coronary lesions: the randomized ROTAXUS (Rotational Atherectomy Prior to Taxus Stent Treatment for Complex Native Coronary Artery Disease) trial. JACC Cardiovasc Interv 6(1):10-19

25. Ali ZA et al (2017) Optical coherence tomography characterization of coronary lithoplasty for treatment of calcified lesions: first description. JACC Cardiovasc Imaging 10(8):897-906

26. Brinton TJ et al (2019) Feasibility of shockwave coronary intravascular lithotripsy for the treatment of calcified coronary stenoses. Circulation 139(6):834-836

27. Watkins S et al (2019) Intravascular lithotripsy to treat a severely underexpanded coronary stent. Eurolntervention 15(1):124-125

28. Lassen JF et al (2018) Percutaneous coronary intervention for the left main stem and other bifurcation lesions: 12th consensus document from the European Bifurcation Club. EuroIntervention 13(13):1540-1553

29. NefHMetal (2016) Bifurcation lesions: how should they be assessed and treated? Herz 41(7):572-578

30. Fajadet J, Capodanno D, Stone GW (2018) Management of left main disease: an update. Eur HeartJ.https://doi.org/10.1093/eurheartj/ehy238

31. Galassi AR et al (2019) Percutaneous recanalisation of chronic total occlusions: 2019 consensus document from the EuroCTO Club. Eurolntervention 15(2):198-208

32. Morino $Y$ et al (2011) Predicting successful guidewire crossing through chronic total occlusion of native coronary lesions within 30 minutes: the J-CTO (Multicenter CTO Registry in Japan) score as a difficulty grading and time assessment tool. JACC Cardiovasc Interv 4(2):213-221

33. Oreglia JA et al (2018) Dual lumen microcatheters for complex percutaneous coronary interventions. Cardiovasc Revasc Med 19(3 Pt A):298-305

34. Maeremans J et al (2016) The hybrid algorithm for treating chronic total occlusions in Europe: the RECHARGE registry. J Am Coll Cardiol 68(18):1958-1970

35. Albiero R et al (2004) Cutting balloon versus conventional balloon angioplasty for the treatment of in-stent restenosis: results of the restenosis cutting balloon evaluation trial (RESCUT). J Am Coll Cardiol 43(6):943-949

36. Giacoppo D et al (2020) Drug-coated balloon angioplasty versus drug-eluting stent implantation in patients with coronary stent restenosis. J Am Coll Cardiol 75(21):2664-2678

37. Leborgne $L$ et al (2003) Effect of direct stenting on clinical outcome in patients treated with percutaneous coronary intervention on saphenous vein graft. Am Heart J 146(3):501-506

38. Okabe T et al (2008) Can direct stenting in selected saphenous vein graft lesions be considered an alternative to percutaneous intervention with a distal protection device? Catheter Cardiovasc Interv 72(6):799-803

39. Baim DS et al (2002) Randomized trial of a distal embolic protection device during percutaneous intervention of saphenous vein aorto-coronary bypass grafts. Circulation 105(11):1285-1290

40. Brennan JMet al (2015) Three-year outcomes associated with embolic protection in saphenous vein graft intervention: results in 49325 senior patients in the Medicare-linked National Cardiovascular Data Registry CathPCI Registry. Circ Cardiovasc Interv 8(3):e1403

41. Paul TK et al (2017) Outcomes of saphenous vein graft intervention with and without embolic protection device: a comprehensive review and meta-analysis. Circ Cardiovasc Interv. https://doi. org/10.1161/CIRCINTERVENTIONS.117.005538

42. Ng AK, Jim M-H (2016) Percutaneous coronary intervention for bifurcation: how can we outperform the provisional strategy? Clin Cardiol 39(11):684-691

43. De MariaGL, Scarsini R,Banning AP (2019) Management of calcific coronary artery lesions: is it time to change our interventional therapeutic approach? JACC Cardiovasc Interv 12(15):1465-1478

44. Genereux P et al (2017) Two-year outcomes after percutaneous coronary intervention of calcified lesions with drug-eluting stents. Int J Cardiol 231:61-67

45. Collet JP et al (2020) ESC Guidelines for the management of acute coronary syndromes in patients presenting without persistent STsegment elevation. Eur Heart J. https://doi.org/10 1093/eurheartj/ehaa575

46. Ibanez B et al (2018) 2017 ESC Guidelines for the management of acute myocardial infarction in patients presenting with ST-segment elevation: The Task Force for the management of acute myocardial infarction in patients presenting with ST-segment elevation of the European Society of Cardiology (ESC). Eur Heart J39(2):119-177

47. Knuuti J et al (2019) 2019 ESC Guidelines for the diagnosis and management of chronic coronary syndromes. Eur Heart J. https://doi.org/10.1093/ eurheartj/ehz425

48. Jolicoeur EM et al (2013) A phase II, shamcontrolled, double-blinded study testing the safety and efficacy of the coronary sinus reducer in 
patients with refractory angina: study protocol for a randomized controlled trial. Trials 14:46

49. Gallone $\mathrm{G}$ et al (2019) Coronary sinus reducer implantation to reduce the ischemic burden in refractory angina. JACC Cardiovasc Interv 12(2):e11-e13

50. Giannini F et al (2019) Patterns of regional myocardial perfusion following coronary sinus reducer implantation. Circ Cardiovasc Imaging 12(9): $\mathrm{e} 9148$

51. Parisi AF, Folland ED, Hartigan P (1992) A comparison of angioplasty with medical therapy in the treatment of single-vessel coronary artery disease. N Engl J Med 326(1):10-16

52. Pepine CJ et al (1994) The Asymptomatic Cardiac Ischemia Pilot(ACIP) study: design of a randomized clinical trial, baseline data and implications for a long-term outcome trial. J Am Coll Cardiol 24(1):1-10

53. RITA-2 trial participants (1997) Coronary angioplasty versus medical therapy for angina: the second Randomised Intervention Treatment of Angina (RITA-2) trial. Lancet 350(9076):461-468

54. Bucher HC et al (2000) Percutaneous transluminal coronary angioplasty versus medical treatment for non-acute coronary heart disease: metaanalysis of randomised controlled trials. BMJ 321(7253):73-77

55. Pfisterer M (2004) Long-term outcome in elderly patients with chronic angina managed invasively versus by optimized medical therapy: four-year follow-up of the randomized Trial of Invasive versus Medical therapy in Elderly patients (TIME). Circulation 110(10):1213-1218

56. Windecker $S$ et al (2014) Revascularisation versus medical treatment in patients with stable coronary artery disease: network meta-analysis. BMJ 348:93859

57. Bangalore $S$ et al (2020) Routine revascularization versus initial medical therapy for stable ischemic heart disease: a systematic review and meta-analysis of randomized trials. Circulation 142(9):841-857

58. Al-Lamee R et al (2018) Percutaneous coronary intervention in stable angina (ORBITA): a double-blind, randomised controlled trial. Lancet 391(10115):31-40

59. De Bruyne B et al (2012) Fractional flow reserveguided $\mathrm{PCl}$ versus medical therapy in stable coronary disease. N Engl J Med 367(11):991-1001

60. Xaplanteris P et al (2018) Five-year outcomes with $\mathrm{PCl}$ guided by fractional flow reserve. $\mathrm{N}$ Engl $\mathrm{J}$ Med 379(3):250-259

61. Boden WE et al (2007) Optimal medical therapy with or without $\mathrm{PCl}$ for stable coronary disease. N Engl J Med 356(15):1503-1516

62. Shaw LJ et al (2008) Optimal medical therapy with or without percutaneous coronary intervention to reduce ischemic burden: results from the Clinical Outcomes Utilizing Revascularization and Aggressive Drug Evaluation (COURAGE) trial nuclear substudy. Circulation 117(10):1283-1291

63. Maron DJ etal (2020) Initial invasive or conservative strategy for stable coronary disease. N Engl J Med 382(15):1395-1407

64. De Bruyne B et al (2014) Fractional flow reserveguided $\mathrm{PCl}$ for stable coronary artery disease. NEngl J Med 371(13):1208-1217

65. Garcia S et al (2013) Outcomes after complete versus incomplete revascularization of patients with multivessel coronary artery disease: a metaanalysis of 89,883 patients enrolled in randomized clinical trials and observational studies. J Am Coll Cardiol 62(16):1421-1431
66. Farooq V et al (2013) Quantification of incomplete revascularization and its association with five-yea mortality in the synergy between percutaneous coronary intervention with taxus and cardiac surgery (SYNTAX) trial validation of the residual SYNTAX score. Circulation 128(2):141-151

67. Park SJ et al (2015) Trial of everolimus-eluting stents or bypass surgery for coronary disease. NEngl J Med 372(13):1204-1212

68. Head SJ et al (2018) Mortality after coronary artery bypass grafting versus percutaneous coronary intervention with stenting for coronary artery disease: a pooled analysis of individual patient data. Lancet 391(10124):939-948

69. Tonino PA etal (2009) Fractional flow reserveversus angiography for guiding percutaneous coronary intervention. N Engl J Med 360(3):213-224

70. Gaede L et al (2019) Coronary angiography with pressure wire and fractional flow reserve: state of the art in the diagnosis of coronary stenosis. Dtsch Arztebl Int 116(12):205-211

71. Ahn JM et al (2015) Randomized trial of stents versus bypass surgery for left main coronary artery disease: 5-year outcomes of the PRECOMBAT study. J Am Coll Cardiol 65(20):2198-2206

72. Makikallio T et al (2016) Percutaneous coronary angioplasty versus coronary artery bypass grafting in treatment of unprotected left main stenosis (NOBLE): a prospective, randomised, open-label, non-inferiority trial. Lancet 388(10061):2743-2752

73. Mohr FW et al (2013) Coronary artery bypass graft surgery versus percutaneous coronary intervention in patients with three-vessel disease and left main coronary disease: 5 -year follow-up of the randomised, clinical SYNTAX trial. Lancet 381(9867):629-638

74. Stone GW et al (2016) Everolimus-eluting stents or bypass surgery for left main coronary artery disease. N Engl J Med 375(23):2223-2235

75. Giacoppo D et al (2017) Percutaneous coronary intervention vs coronary artery bypass grafting in patients with left main coronary artery stenosis: a systematic review and meta-analysis. JAMA Cardiol 2(10):1079-1088

76. Mitra AK, Agrawal DK (2006) In stent restenosis: bane of the stent era. JClin Pathol 59(3):232-239

77. Brener SJ et al (2004) Propensity analysis of long-term survival after surgical or percutaneous revascularization in patients with multivessel coronary artery disease and high-risk features. Circulation 109(19):2290-2295

78. Hoffman SN et al (2003) A meta-analysis of randomized controlled trials comparing coronary artery bypass graft with percutaneous transluminal coronary angioplasty: one- to eight-year outcomes. J Am Coll Cardiol 41 (8):1293-1304

79. Farkouh ME et al (2012) Strategies for multivessel revascularization in patients with diabetes. N Engl JMed 367(25):2375-2384

80. Nashef SA et al (2012) EuroSCORE II. Eur J Cardiothorac Surg 41(4):734-744 (discussion 744-5)

81. Shahian DM et al (2009) The Society of Thoracic Surgeons 2008 cardiac surgery risk models: part 1 - coronary artery bypass grafting surgery. Ann Thorac Surg 88(1 Suppl):S2-S22

82. Velicki L et al (2014) Clinical performance of the EuroSCORE II compared with the previous EuroSCORE iterations. Thorac Cardiovasc Surg 62(4):288-297

83. Savage MP et al (1997) Stent placement compared with balloon angioplasty for obstructed coronary bypass grafts. N Engl J Med 337(11):740-747
84. Al-Lamee R et al (2010) Clinical and angiographic outcomes after percutaneous recanalization of chronic total saphenous vein graft occlusion using modern techniques. Am J Cardiol 106(12):1721-1727

85. Galassi AR et al (2016) Appropriateness of percutaneous revascularization of coronary chronic total occlusions: an overview. Eur Heart J 37(35):2692-2700

86. Jeroudi OM et al (2014) Prevalence and management of coronary chronic total occlusions in a tertiary Veterans Affairs hospital. Catheter Cardiovasc Interv 84(4):637-643

87. Christakopoulos GE et al (2015) Meta-analysis of clinical outcomes of patients who underwent percutaneous coronary interventions for chronic total occlusions. Am J Cardiol 115(10):1367-1375

88. Lee SW et al (2019) Randomized trial evaluating percutaneous coronary intervention for the treatment of chronic total occlusion. Circulation 139(14):1674-1683

89. Werner GS et al (2018) A randomized multicentre trial to compare revascularization with optimal medical therapy for the treatment of chronic total coronary occlusions. Eur Heart J39(26):2484-2493

90. Morino $Y$ et al (2011) Predicting successful guidewire crossing through chronic total occlusion of native coronary lesions within 30 minutes: the J-CTO (Multicenter CTO Registry in Japan) score as a difficulty grading and time assessment tool. JACC Cardiovasc Interv 4(2):213-221

91. Moscucci M, Eagle KA (2004) Coronary revascularization before noncardiac surgery. $\mathrm{N}$ Engl J Med 351:2861-2863

92. Rissanen TT et al (2019) Drug-coated balloon for treatment of de-novo coronary artery lesions in patients with high bleeding risk (DEBUT): a singleblind, randomised, non-inferiority trial. Lancet 394(10194):230-239

93. Urban P et al (2015) Polymer-free drug-coated coronary stents in patients at high bleeding risk. NEngl J Med 373(21):2038-2047

94. Varenne $O$ et al (2018) Drug-eluting stents in elderly patients with coronary artery disease (SENIOR): a randomised single-blind trial. Lancet 391(10115):41-50

95. Jolly SS et al (2011) Radial versus femoral access for coronary angiography and intervention in patients with acute coronary syndromes (RIVAL): a randomised, parallel group, multicentre trial. Lancet 377(9775):1409-1420

96. Rao SV et al (2014) A registry-based randomized trial comparing radial and femoral approaches in women undergoing percutaneous coronary intervention: the SAFE-PCI for Women (Study of Access Site for Enhancement of $\mathrm{PCl}$ for Women) trial. JACC Cardiovasc Interv 7(8):857-867

97. Valgimigli M et al (2015) Radial versus femoral access in patients with acute coronary syndromes undergoing invasive management: a randomised multicentre trial. Lancet 385(9986):2465-2476

98. Ando G, Capodanno D (2016) Radial access reduces mortality in patients with acute coronary syndromes: results from an updated trial sequential analysis of randomized trials. JACC Cardiovasc Interv 9(7):660-670

99. Valgimigli M et al (2018) Radial versus femoral access and bivalirudin versus unfractionated heparin in invasively managed patients with acute coronary syndrome (MATRIX): final 1-year results of a multicentre, randomised controlled trial. Lancet 392(10150):835-848

100. Le May M (2019) The safety and afficacy of femoral access vs radial access in STEMI. In: ACC Congr 
101. Sgarbossa EB, Birnbaum Y, Parrillo JE (2001) Electrocardiographic diagnosis of acute myocardial infarction: current concepts for the clinician. Am Heart J 141(4):507-517

102. de WinterRJetal (2008) A newECG sign of proximal LAD occlusion. $n$ Engl JMed 359:2071-2073

103. Kurisu S et al (2004) Electrocardiographic features in patients with acute myocardial infarction associated with left main coronary artery occlusion. Heart 90:1059-1060

104. Roffi M et al (2016) 2015 ESC Guidelines for the management of acute coronary syndromes in patients presenting without persistent ST-segment elevation: Task Force for the Management of Acute Coronary Syndromes in Patients Presenting without Persistent ST-Segment Elevation of the European Society of Cardiology (ESC). Eur Heart J 37(3):267-315

105. Thiele $\mathrm{H}$ et al (2012) Optimal timing of invasive angiography in stable non-ST-elevation myocardial infarction: the Leipzig Immediate versus early and late PercutaneouS coronary Intervention triAl in NSTEMI (LIPSIA-NSTEMI Trial). Eur Heart J 33(16):2035-2043

106. Kastrati A et al (2011) Abciximab and heparin versus bivalirudin for non-ST-elevation myocardial infarction. NEngl J Med 365(21):1980-1989

107. Virmani R et al (2006) Pathology of the vulnerable plaque. J Am Coll Cardiol 47(8Suppl):C13-C18

108. Cheruvu PKet al (2007) Frequency and distribution of thin-cap fibroatheroma and ruptured plaques in human coronary arteries: a pathologic study. J Am Coll Cardiol 50(10):940-949

109. Goldstein JA et al (2000) Multiple complex coronary plaques in patients with acute myocardial infarction. NEngl J Med 343(13):915-922

110. KerenskyRAetal (2002) Revisiting the culpritlesion in non-Q-wave myocardial infarction. Results from the VANQWISH trial angiographic core laboratory. J Am Coll Cardiol 39(9):1456-1463

111. Shishehbor MH et al (2007) In unstable angina or non-ST-segmentacute coronarysyndrome, should patients with multivessel coronary artery disease undergo multivessel or culprit-only stenting? J Am Coll Cardiol 49(8):849-854

112. SmitsPCetal (2017) Fractional flow reserve-guided multivessel angioplasty in myocardial infarction. NEngl J Med 376(13):1234-1244

113. Cannon CP et al (2001) Comparison of early invasive and conservative strategies in patients with unstable coronary syndromes treated with the glycoprotein Ilb/Illa inhibitor tirofiban. N Engl J Med 344(25):1879-1887

114. Fox KA et al (2005) 5-year outcome of an interventional strategy in non-ST-elevation acute coronary syndrome: the British Heart Foundation RITA 3 randomised trial. Lancet 366(9489):914-920

115. Wallentin L et al (2000) Outcome at 1 year after an invasive compared with a non-invasive strategy in unstable coronary-artery disease: the FRISC II invasive randomised trial. Lancet 356(9223):9-16

116. Curtis JP et al (2009) All-cause readmission and repeat revascularization after percutaneous coronary intervention in a cohort of medicare patients. J Am Coll Cardiol 54(10):903-907

117. Genereux P et al (2012) Quantification and impact of untreated coronary artery disease after percutaneous coronary intervention: the residual SYNTAX (Synergy Between PCI with Taxus and Cardiac Surgery) score. J Am Coll Cardiol 59(24):2165-2174

118. Ben-Gal Yetal (2010) Surgical versus percutaneous revascularization for multivessel disease in patients with acute coronary syndromes: analysis from the ACUITY (Acute Catheterization and Urgent Intervention Triage Strategy) trial. JACC Cardiovasc Interv3(10):1059-1067

119. Ranasinghe l et al (2011) Risk stratification in the setting of non-ST elevation acute coronary syndromes 1999-2007. Am J Cardiol 108(5):617-624

120. Andersen HR et al (2003) A comparison of coronary angioplasty with fibrinolytic therapy in acute myocardial infarction. $\mathrm{N}$ Engl J Med 349(8):733-742

121. Widimsky Petal (2003) Long distance transportfor primary angioplasty vs immediate thrombolysis in acute myocardial infarction. Final results of the randomized national multicentre trial_-PRAGUE-2. Eur Heart J 24(1):94-104

122. Keeley EC, Boura JA, Grines CL (2003) Primary angioplasty versus intravenous thrombolytic therapy for acute myocardial infarction: a quantitative review of 23 randomised trials. Lancet 361(9351):13-20

123. Zijlstra F et al (1999) Long-term benefit of primary angioplasty as compared with thrombolytic therapy for acute myocardial infarction. N Engl J Med341(19):1413-1419

124. Scholz KH et al (2018) Impact of treatment delay on mortality in ST-segment elevation myocardial infarction (STEMI) patients presenting with and without haemodynamic instability: results from the German prospective, multicentre FITT-STEMI trial. Eur Heart J39(13):1065-1074

125. Borgia $F$ et al (2010) Early routine percutaneous coronary intervention after fibrinolysis vs. standard therapy in ST-segment elevation myocardial infarction: a meta-analysis. Eur Heart $J$ 31(17):2156-2169

126. D'Souza SP et al (2011) Routine early coronary angioplasty versus ischaemia-guided angioplasty after thrombolysis in acute ST-elevation myocardial infarction: a meta-analysis. Eur Heart J 32(8):972-982

127. Hochman JS et al (2006) Coronary intervention for persistent occlusion after myocardial infarction. NEngl J Med 355(23):2395-2407

128. Engstrom Tet al (2015) Complete revascularisation versus treatment of the culprit lesion only in patients with ST-segment elevation myocardial infarction and multivessel disease (DANAMI-3PRIMULTI): an open-label, randomised controlled trial. Lancet 386(9994):665-671

129. Kelly DJ et al (2013) Complete Versus culprit-Lesion only PRimary PCI Trial (CVLPRIT): a multicentre trial testing management strategies when multivessel disease is detected at the time of primary $\mathrm{PCl}$ : rationale and design. Eurolntervention 8(10):1190-1198

130. WaldDS etal (2013) Randomized trial of preventive angioplasty in myocardial infarction. N Engl J Med 369(12):1115-1123

131. Mehta SR et al (2019) Complete revascularization with multivessel $\mathrm{PCl}$ for myocardial infarction. NEngl J Med 381(15):1411-1421

132. Frobert $O$ et al (2013) Thrombus aspiration during ST-segment elevation myocardial infarction. NEngl J Med 369(17):1587-1597

133. Jolly SS et al (2015) Randomized trial of primary PCI with or without routine manual thrombectomy. NEngl J Med 372(15):1389-1398

134. Jolly SS et al (2015) Stroke in the TOTAL trial: a randomized trial of routine thrombectomy vs. percutaneous coronary intervention alone in ST elevation myocardial infarction. Eur Heart J 36(35):2364-2372

135. Holmes DR Jr. et al (1999) Cardiogenic shock in patients with acute ischemic syndromes with and without ST-segment elevation. Circulation 100(20):2067-2073

136. Babaev A et al (2005) Trends in management and outcomes of patients with acute myocardial infarction complicated by cardiogenic shock. JAMA 294(4):448-454

137. Thiele $\mathrm{H}$ et al (2017) PCl strategies in patients with acute myocardial infarction and cardiogenic shock. NEngl J Med 377(25):2419-2432

138. Thiele $\mathrm{H}$ et al (2012) Intraaortic balloon support for myocardial infarction with cardiogenic shock. NEngl JMed 367(14):1287-1296

139. Hayes SN et al (2018) Spontaneous coronary artery dissection: current state of the science: a scientific statement from the American Heart Association. Circulation 137(19):e523-e557

140. Saw J et al (2017) Spontaneous coronary artery dissection:clinical outcomesand risk of recurrence. JAm Coll Cardiol 70(9):1148-1158

141. Saw J et al (2019) Canadian spontaneous coronary artery dissection cohort study: in-hospital and 30day outcomes. Eur Heart J 40(15):1188-1197

142. Nef HM et al (2017) Medikamentenfreisetzende Koronarstents/-scaffolds und medikamentenbeschichtete Ballonkatheter. Kardiologe 12(1):26-52

143. Alfonso $F$ et al (2018) 3-year clinical followup of the RIBS IV clinical trial: a prospective randomized study of drug-eluting balloons versus everolimus-eluting stents in patients with in-stent restenosis in coronary arteries previously treated with drug-eluting stents. JACC Cardiovasc Interv 11(10):981-991

144. Hamm CW et al (2020) A multicentre, randomised controlled clinical study of drug-coated balloons for the treatment of coronary in-stent restenosis. Eurolntervention 16(4):e328-e334

145. Savage MP et al (1995) Effect of thromboxane A2 blockade on clinical outcome and restenosis after successful coronary angioplasty. Multi-Hospital Eastern Atlantic Restenosis Trial (M-HEART II). Circulation 92(11):3194-3200

146. Antithrombotic Trialists' Collaboration (2002) Collaborative meta-analysis of randomised trials of antiplatelet therapy for prevention of death, myocardial infarction, and stroke in high risk patients. BMJ 324(7329):71-86

147. Patrono Cet al (2004) Expert consensus document on the use of antiplatelet agents. The task force on the use of antiplatelet agents in patients with atherosclerotic cardiovascular disease of the European society of cardiology. Eur Heart J 25(2):166-181

148. Steg PG et al (2013) Effect of cangrelor on periprocedural outcomes in percutaneous coronary interventions: a pooled analysis of patient-level data. Lancet 382(9909):1981-1992

149. Harrington RA et al (2009) Platelet inhibition with cangrelor in patients undergoing $\mathrm{PCl}$. N Engl J Med 361(24):2318-2329

150. Kaluski E et al (2000) Minimal heparinization in coronary angioplasty - how much heparin is really warranted? Am J Cardiol 85(8):953-956

151. Montalescot $\mathrm{G}$ et al (2006) Enoxaparin versus unfractionated heparin in elective percutaneous coronary intervention. $\mathrm{N}$ Engl J Med 355(10):1006-1017

152. Kastrati A et al (2008) Bivalirudin versus unfractionated heparin during percutaneous coronary intervention. NEngl J Med 359(7):688-696

153. Wiviott SD et al (2007) Prasugrel versus clopidogrel in patients with acute coronary syndromes. N Eng JMed 357(20):2001-2015 
154. Wallentin L et al (2009) Ticagrelor versus clopidogrel in patients with acute coronary syndromes. NEnglJMed 361(11):1045-1057

155. Montalescot $\mathrm{G}$ et al (2014) Prehospital ticagrelor in ST-segment elevation myocardial infarction. NEngl J Med 371(11):1016-1027

156. Montalescot $\mathrm{G}$ et al (2013) Pretreatment with prasugrel in non-ST-segmentelevation acute coronary syndromes. N EnglJ Med 369(11):999-1010

157. Schupke $S$ et al (2019) Ticagrelor or prasugrel in patients with acute coronary syndromes. N Engl J Med 381(16):1524-1534

158. ten Berg JM et al (2010) Effect of early, pre-hospital initiation of high bolus dose tirofiban in patients with ST-segment elevation myocardial infarction on short-and long-term clinical outcome. J Am Coll Cardiol 55(22):2446-2455

159. Montalescot $\mathrm{G}$ et al (2011) Intravenous enoxaparin or unfractionated heparin in primary percutaneous coronary intervention for ST-elevation myocardial infarction: the international randomised openlabel ATOLL trial. Lancet 378(9792):693-703

160. Silvain $J$ et al (2012) Efficacy and safety of enoxaparin versus unfractionated heparin during percutaneous coronary intervention: systematic review and meta-analysis. BMJ344:e553

161. Capodanno D et al (2016) Bivalirudin versus heparin with or without glycoprotein Ilb/llla inhibitors in patients with STEMI undergoing primary $\mathrm{PCl}$ : an updated meta-analysis of 10,350 patients from five randomized clinical trials. Eur Heart J Acute Cardiovasc Care 5(3):253-262

162. Stone GW et al (2006) Bivalirudin for patients with acute coronary syndromes. N Engl J Med 355(21):2203-2216

163. Valgimigli M, MATRIX investigators (2014) Design and rationale for the Minimizing Adverse haemorrhagic events by TRansradial access site and systemic Implementation of angioX program. Am Heart J 168(6):838-845

164. Shahzad A et al (2014) Unfractionated heparin versus bivalirudin in primary percutaneous coronary intervention (HEAT-PPCI): an open-label, single centre, randomised controlled trial. Lancet 384(9957):1849-1858

165. LeonardiSetal (2016) Bivalirudin or unfractionated heparin in patients with acute coronary syndromes managed invasively with and without ST elevation (MATRIX): randomised controlled trial. BM i4935:354

166. Cannon CP et al (2017) Dual antithrombotic therapy with dabigatran after $\mathrm{PCl}$ in atrial fibrillation. N Engl JMed 377(16):1513-1524

167. Gibson CM et al (2016) Prevention of bleeding in patients with atrial fibrillation undergoing $\mathrm{PCl}$. NEngl J Med 375(25):2423-2434

168. Lopes RD et al (2019) Antithrombotic therapy after acute coronary syndrome or PClin atrial fibrillation. NEngl J Med 380(16):1509-1524

169. Dash D (2013) Complications of coronary intervention: abrupt closure, dissection, perforation. Heart Asia 5(1):61-65

170. Giannini F et al (2018) A practical approach to the management of complications during percutaneous coronary intervention. JACC Cardiovasc Interv 11(18):1797-1810

171. Albertal $M$ et al (2001) Uncomplicated moderate coronary artery dissections after balloon angioplasty: good outcome without stenting. Heart 86(2):193-198

172. Huber MS et al (1991) Use of a morphologic classification to predict clinical outcome after dissection from coronary angioplasty. Am J Cardiol 68(5):467-471

\section{Manual of the working group on interventional cardiology (AGIK) of the German Cardiac Society (DGK). Part 2: "Performance of percutaneous coronary interventions"}

This manual addressing the interventional treatment (part 2) is a recommendation for interventional cardiologists. It displays the latest evidence in interventional cardiology. Each chapter was designed to focus on the everyday practicability and to provide decision support for cardiologists in the daily clinical routine of a catheter laboratory. Despite the practical advice by many experts, this manual cannot replace the medical evaluation of each individual patient and thus an adaptation of the diagnostics or treatment is necessary.

\section{Keywords}

Catheter laboratory - Cardiovascular disease - Acute coronary syndrome - Decision support . Cardiac catheterization

173. Gibson CM et al (2000) Relationship of TIMI myocardial perfusion grade to mortality after administration of thrombolytic drugs. Circulation 101(2):125-130

174. Muller $\mathrm{O}$ et al (2008) Management of two major complications in the cardiac catheterisation laboratory: the no-reflow phenomenon and coronary perforations. Eurolntervention 4(2):181-183

175. Niccoli G et al (2009) Myocardial no-reflow in humans. J Am Coll Cardiol 54(4):281-292

176. Ross AM et al (2005) A randomized, doubleblinded, placebo-controlled multicenter trial of adenosine as an adjunct to reperfusion in the treatment of acute myocardial infarction (AMISTAD-II).J Am Coll Cardiol 45(11):1775-1780

177. Farouque HM et al (2005) Risk factors for the development of retroperitoneal hematoma after percutaneous coronary intervention in the era of glycoprotein Ilb/llla inhibitors and vascular closure devices. J Am Coll Cardiol 45(3):363-368

178. Maluenda $G$ et al (2012) Retroperitoneal hemorrhage after percutaneous coronary intervention in the current practice era: clinical outcomes and prognostic value of abdominal/pelvic computed tomography. Catheter Cardiovasc Interv 80(1):29-36

179. Tiroch KA et al (2008) Risk predictors of retroperitoneal hemorrhage following percutaneous coronaryintervention. Am J Cardiol 102(11):1473-1476

180. Kinnaird T et al (2016) Incidence, determinants, and outcomes of coronary perforation during percutaneous coronary intervention in the United Kingdom between 2006 and 2013: an analysis of 527121 cases from the British Cardiovascular Intervention Society database. Circ Cardiovasc Interv 9:8. https://doi.org/10.1161/ CIRCINTERVENTIONS.115.003449

181. Shimony A et al (2011) Coronary artery perforation during percutaneous coronary intervention: a systematic review and meta-analysis. Can J Cardiol 27(6):843-850

182. Dippel EJ et al (2001) Coronary perforation during percutaneous coronary intervention in the era of abciximab platelet glycoprotein IIb/IIla blockade: an algorithm for percutaneous management. Catheter Cardiovasc Interv 52(3):279-286

183. Ellis SG et al (1988) Angiographic and clinical predictors of acute closure after native vessel coronary angioplasty. Circulation 77(2):372-379

184. Ellis SG et al (1994) Increased coronary perforation in the new device era. Incidence, classifica- tion, management, and outcome. Circulation 90(6):2725-2730

185. Brilakis ES et al (2005) Incidence, retrieval methods, and outcomes of stent loss during percutaneous coronary intervention: a large single-center experience. Catheter Cardiovasc Interv 66(3):333-340

186. Colkesen AY et al (2007) Coronary and systemic stent embolization during percutaneous coronary interventions: a single center experience. Int Heart J48(2):129-136

187. Alomar ME et al (2013) Stent loss and retrieval during percutaneous coronary interventions: a systematic review and meta-analysis. J Invasive Cardiol 25(12):637-641

188. Meller J et al (1976) Coronary artery dissection-a complication of cardiac catheterization without sequelae: case report and review of the literature. Cathet Cardiovasc Diagn 2(3):301-308

189. Gomez-Moreno Set al (2006) latrogenic dissection of the ascending aorta following heart catheterisation: incidence, management and outcome. Eurolntervention 2(2): 197-202

190. Dunning DWetal (2000) latrogenic coronary artery dissections extending into and involving the aortic root. Catheter Cardiovasc Interv 51(4):387-393

191. Röther J, Laufs U, Böhm M, Willems S, Scheller B, Borggrefe M, Darius H, Endres M, Zeymer U, Diener HC, Grond M, Hacke W, Forsting M, Schumacher M, Hennerici M (2009) Konsensuspapier „Peri- und postinterventioneller Schlaganfall bei Herzkatheterprozeduren“. Kardiologe (3):375-387

192. Aggarwal A et al (2009) Incidence and predictors of stroke associated with percutaneous coronary intervention. Am J Cardiol 104(3):349-353

193. Dukkipati $S$ et al (2004) Characteristics of cerebrovascular accidents after percutaneous coronary interventions. J Am Coll Cardiol 43(7):1161-1167

194. München O (2010) Urt.v. 18.11.2010-1 U 5334/09, GesR 2011, 235, $236=$ juris Nr. 35, 37, 44, 45: Bei Aufklärung durch Assistenzarzt

195. Frahm, Nixdorf, Walter (2013) 5. Aufl., Rz. 215 zu § 630 ell 1 Nr. 1 BGB

196. Martis, Winkhart (2014) 4. Aufl., Rz. A 1757 ff. und Rz.P 44, P 48

\section{Weiterführende Literatur}

197. Montalescot G et al (2013) 2013 ESC guidelines on the management of stable coronary artery disease: 


\section{Curriculum}

the Task Force on the management of stable coronary artery disease of the European Society of Cardiology. Eur Heart J 34(38):2949-3003

198. Campeau L (1976) Letter: grading of angina pectoris. Circulation 54(3):522-523

199. New York Heart Association (2016) NYHA-Clas sification. https://manual.jointcommission.org/ releases/TJC2016A/DataElem0439.html. Zugegriffen: 12. Dez. 2019

200. Sianos G et al (2005) The SYNTAX Score: an angiographic tool grading the complexity of coronary artery disease. Eurolntervention 1(2):219-227

201. Medina A, Suarez de Lezo J, Pan M (2006) A new classification of coronary bifurcation lesions. Rev Esp Cardiol 59(2):183

202. Mehran R et al (1999) Angiographic patterns of in-stent restenosis: classification and implications for long-term outcome. Circulation 100(18):1872-1878

203. Rentrop KP et al (1985) Changes in collateral channel filling immediately after controlled coronary artery occlusion by an angioplasty balloon in human subjects. J Am Coll Cardiol 5(3):587-592

\section{Affiliations}

'Medizinische Klinik I, Kardiologie und Angiologie, Universitätsklinikum Gießen und Marburg GmbH Gießen, Deutschland; ' ${ }^{2}$ Medizinische Klinik 2, Friedrich-Alexander-Universität Erlangen Nürnberg, Erlangen, Deutschland; ${ }^{3}$ Herzklinik Ulm, Ulm, Deutschland; ${ }^{4}$ Medizinische Klinik I, Helios Klinikum Krefeld, Krefeld, Deutschland; ${ }^{5}$ Universität Witten/Herdecke, Witten, Deutschland; ${ }^{6}$ Herz-KreislaufZentrum, Universitätsklinik für Innere Medizin - Kardiologie, Klinikum Oldenburg, Oldenburg, Deutschland; ' 'Zentrum für Kardiologie - Kardiologie I, Mainz und DZHK Standort Rhein-Main, Universitätsmedizin Mainz, Frankfurt am Main, Deutschland; ${ }^{8} \mathrm{Klinik}$ für Kardiologie und allgemeine Innere Medizin, Städtisches Klinikum Solingen gemeinnützige $\mathrm{GmbH}$, Solingen, Deutschland; ${ }^{9} \mathrm{Klinik}$ für Innere Medizin, Universitätsklinikum Heidelberg, Heidelberg, Deutschland; ${ }^{10}$ Abteilung für Kardiologie, Bad Nauheim und DZHK Standort Rhein-Main, Campus der JLU, Kerkhoff Bad Nauheim, Frankfurt am Main, Deutschland; "CCB - Cardioangiologisches Centrum Bethanien, Frankfurt am Main, Deutschland; ${ }^{12}$ Medizinische Klinik und Poliklinik I, Klinikum der Universität München, München, Deutschland; ${ }^{13}$ Klinik für Innere Medizin 8, Schwerpunkt Kardiologie, Universitätsklinik der Paracelsus Medizinischen Privatuniversität, Nürnberg, Deutschland; ${ }^{14}$ Klinik für Kardiologie und Angiologie, Contilia Herz- und Gefäßzentrum, Essen, Deutschland; ${ }^{15}$ Kardiologische Praxisklinik Ludwigshafen, Ludwigshafen, Deutschland; ${ }^{16}$ Medizinische Klinik und Poliklinik, Universitätsklinikum Würzburg, Würzburg, Deutschland; ${ }^{17}$ Klinik für Herz- und Kreislauferkrankungen, Deutsches Herzzentrum München, München, Deutschland; ${ }^{18}$ Medizinische Klinik B, Klinikum der Stadt Ludwigshafen am Rhein gemeinnützige GmbH, Ludwigshafen, Deutschland; ${ }^{19}$ Kommission für Klinische Kardiovaskuläre Medizin, Deutschen Gesellschaft für Kardiologie - Herz- und Kreislaufforschung e.V., Düsseldorf, Deutschland; ${ }^{20}$ Klinik für Kardiologie, Angiologie und Nephrologie, Universitätsklinik Frankfurt, Frankfurt, Deutschland; ${ }^{21}$ Klinik für Innere Medizin I, St.-Johannes-Hospital Dortmund, Dortmund, Deutschland 